\title{
The Total Synthesis of Ustiloxin D and Considerations on the Origin of Selectivity of the Asymmetric Allylic Alkylation
}

Andrew M. Sawayama, ${ }^{1}$ Hiroko Tanaka, ${ }^{1}$ and Thomas J. Wandless ${ }^{2}$

${ }^{1}$ Department of Chemistry, Stanford University, Stanford, CA 94305

${ }^{2}$ Department of Molecular Pharmacology, Stanford University, Stanford, CA 94305

Table of Contents

S2.......Figure $\mathrm{S} 1$

S3.......Experimental Procedures, $\mathbf{5}$

S4......6, 9

S5.......10, 11

S6......12, 13

S7......4, 16

S8......17, 21, 23

S9......25, 27

S10......29, 30

S11....31, 32, 73

S12....34, 40

S13....41b, 42d

S14....43

S15....44

S16....45, 46

S17....47

S18....52

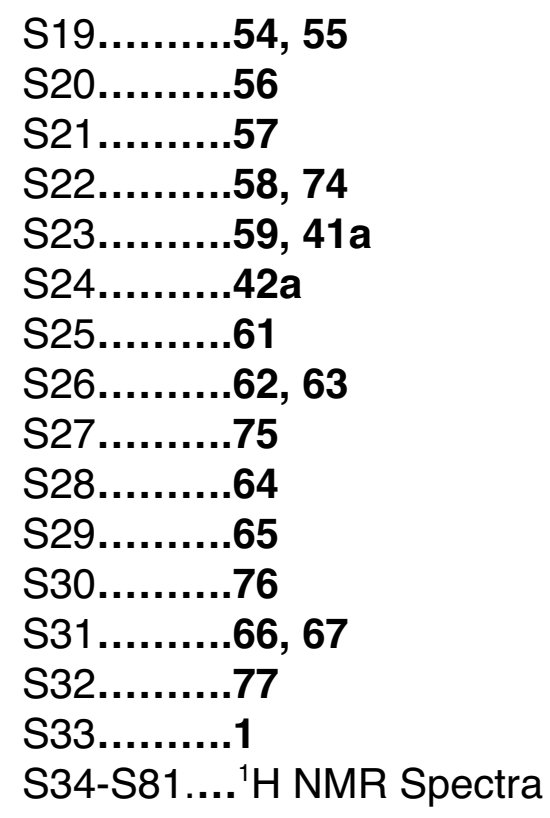

S20..........56

S21..........57

S22..........58, 74

S23..........59, 41a

S24...........42a

S25..........61

S26.........62, 63

S27..........75

S28...........64

S29..........65

S30...........76

S31..........66, 67

S32...........77

S34-S81.... ${ }^{1} \mathrm{H}$ NMR Spectra 


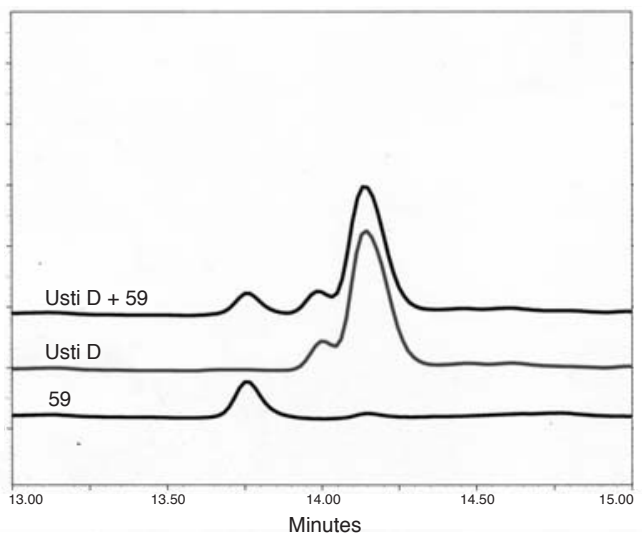

Figure S1. HPLC Traces of Natural Ustiloxin D and 59 
Experimental Procedures. All reactions were carried out in flame-dried glassware sealed with a rubber septum under an argon atmosphere. Liquids and solutions were transferred via syringe or stainless steel cannula. The reaction solvents THF, $\mathrm{Et}_{2} \mathrm{O}$, toluene and benzene were distilled over sodium metal, $\mathrm{CH}_{2} \mathrm{Cl}_{2}, \mathrm{CH}_{3} \mathrm{CN}, \mathrm{NEt}_{3}, \mathrm{iPr}_{2} \mathrm{NEt}$ and pyridine were distilled over calcium hydride and anhydrous DMF in AcroSeal bottles were used without further purification. Flash chromatography was carried out using ICN silica gel 32-63 (230-400 mesh). Analytical thin layer chromatography (TLC) was performed using EM $0.25 \mathrm{~mm}$ silica gel $60 \mathrm{~F}_{254}$ glass plates. TLC plates were visualized using UV fluorescence and by one or more of the following: ceric ammonium molybdate, potassium permanganate, ninhydrin, vanillin and iodine. Optical rotation was measured on a digital polarimeter using a sodium lamp at the indicated temperature.

Infrared spectra were recorded on a FT-IR spectrometer and are reported in wavenumbers $\left(\mathrm{cm}^{-1}\right) .{ }^{1} \mathrm{H}$ NMR spectra were recorded on a $500 \mathrm{MHz}$ and $400 \mathrm{MHz}$ spectrometer and are reported in ppm using the solvent resonance as an internal standard $\left(\mathrm{CHCl}_{3}=7.26 \mathrm{ppm}\right.$, $\left.\mathrm{CD}_{2} \mathrm{HOD}=3.30 \mathrm{ppm}, \mathrm{CD}_{2} \mathrm{HS}(\mathrm{O}) \mathrm{CD}_{3}=2.49 \mathrm{ppm}, \mathrm{DOH}=4.67 \mathrm{ppm}\right)$. Data are reported as: $\delta$ shift ( $\mathrm{s}=$ singlet, $\mathrm{d}=$ doublet, $\mathrm{t}=$ triplet, $\mathrm{q}=$ quartet, $\mathrm{m}=$ =multiplet, $\mathrm{b}=$ broad, $J=$ coupling constant in $\mathrm{Hz}$, integration). Proton decoupled ${ }^{13} \mathrm{C}$ NMR spectra were recorded on a 500 $\mathrm{MHz}$ and $400 \mathrm{MHz}$ spectrometer and are reported in ppm using the solvent resonance as an internal standard $\left(\mathrm{CDCl}_{3}=77.0 \mathrm{ppm}, \mathrm{CD}_{3} \mathrm{OD}=49.0 \mathrm{ppm}, \mathrm{DMSO}=39.5 \mathrm{ppm}, 1,4-\right.$ dioxane $=66.5 \mathrm{ppm})$.

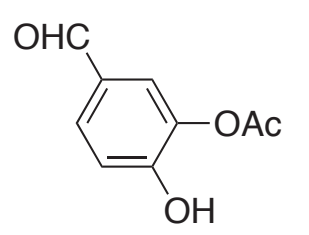

3-Acetoxy-4-hydroxybenzaldehyde (5). 3,4-Dihydroxybenzaldehyde (3.9 g, 28 mmol, 1 equiv, pretreated with activated charcoal in methanol-ethyl acetate mixture, filtered through celite and solvents removed) was suspended in $39 \mathrm{~mL}$ THF and cooled to $0{ }^{\circ} \mathrm{C}$. Sodium hydroxide solution $(35 \mathrm{~mL}, 70 \mathrm{mmol}, 2.5$ equiv, $2 \mathrm{~N}$ solution in water) was added dropwise, followed by a dropwise addition of acetic anhydride $(3.2 \mathrm{~mL}$, $34 \mathrm{mmol}, 1.2$ equiv). The reaction mixture was allowed to stir for $20 \mathrm{~min}$ then it was diluted with ethyl acetate. The reaction was acidified by addition of concentrated hydrochloric acid $(2.5 \mathrm{~mL})$ and $\mathrm{pH} 2.5$ phosphate buffer $(20 \mathrm{~mL})$. THF was removed under reduced pressure, and the reaction mixture was filtered through a pad of celite and rinsed with ethyl acetate. The organic layer was separated and the aqueous layer was extracted twice with ethyl acetate. The combined organic layers were dried over anhydrous sodium sulfate. Solvent was removed under reduced pressure and the crude product was purified by silica gel chromatography. Byproduct 3,4diacetoxybenzaldehyde was eluted using 100/1 chloroform/methanol with $0.5 \%$ triethylamine. Main product fractions were eluted using $70 / 1$ to $50 / 1$ chloroform/methanol, followed by 10/1 chloroform/methanol to elute 3-acetoxy-4hydroxybenzaldehyde 5 (4.38 g, $24.3 \mathrm{mmol}, 86 \%)$ (pale-yellow solid) was obtained along with $503 \mathrm{mg}(2.26 \mathrm{mmol}, 8 \%)$ of 3,4-diacetoxybenzaldehyde and $217 \mathrm{mg}(1.57 \mathrm{mmol}$, $6 \%$ ) of 3,4-dihydroxybenzaldehyde. All spectral properties matched literature values. ${ }^{1}$

\footnotetext{
${ }^{1}$ Buchanan, J. G.; Hill, D. G.; Wightman, R. H.; Boddy, I. K.; Hewitt, B. D. Tetrahedron 1995, 51, 6033.
} 


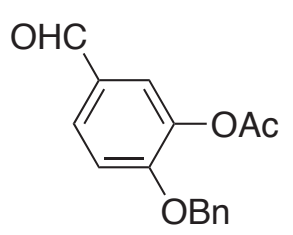

3-Acetoxy-4-benzyloxybenzaldehyde (6). Potassium carbonate (24.55 g, $177.6 \mathrm{mmol}, 4$ equiv) was added to DMF $(250 \mathrm{~mL})$ and cooled to $0{ }^{\circ} \mathrm{C}$. Benzyl iodide was added dropwise $(11.1 \mathrm{~mL}, 88.8$ mmol, 2 equiv) followed by dropwise addition of a solution of 5 ( 8.0 g, $44.4 \mathrm{mmol}$, 1 equiv in $100 \mathrm{~mL}$ DMF) via an addition funnel. An additional $100 \mathrm{~mL}$ DMF was used to rinse the addition funnel. Reaction was complete after $30 \mathrm{~min}$ at which time $\mathrm{pH} 2.5$ buffer was added $(450 \mathrm{~mL})$ followed by water $(500 \mathrm{~mL})$ and then concentrated $\mathrm{HCl}(10 \mathrm{~mL})$ to produce a solution of $\mathrm{pH} \sim 4$. An additional $500 \mathrm{~mL}$ water diluted the mixture and this was extracted with $(4 \times$ $500 \mathrm{~mL}$ ) diethyl ether. These extracts were subsequently dried over anhydrous $\mathrm{Na}_{2} \mathrm{SO}_{4}$ and the volatiles removed under reduced pressure. The crude oil was loaded onto a silica gel column. Excess benzyl iodide was eluted using a gradient of 10/1 to 5/1 hexanes/ethyl acetate, followed by elution with $3 / 1$ to obtain 6 (11.5 g, $42.5 \mathrm{mmol}, 96 \%)$ as a white solid. All spectral properties matched literature values. ${ }^{2}$

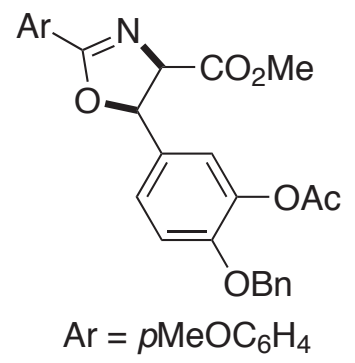

(4R,5R)-5-(3-Acetoxy-4-benzyloxyphenyl)-2-(4-methoxyphenyl)-4,5-dihydrooxazole-4-(carboxylic acid methyl ester) (9). Molecular sieves $\left(3 \AA, 1.24 \mathrm{~g}\right.$, dried overnight in $300{ }^{\circ} \mathrm{C}$ oven), anhydrous sodium sulfate ( $68 \mathrm{mg}, 0.479 \mathrm{mmol}, 0.2$ equiv), 5-methoxy-2-(4-methoxyphenyl)-oxazole 8 (489 mg, $2.38 \mathrm{mmol}$, 1 equiv), 6 (775 mg, $2.87 \mathrm{mmol}, 1.2$ equiv) and (S)-2,2'-bis(3,5di-tert-butyl-2-hydroxybenzilideneamino)-1,1'-binaphthyl aluminum chloride 7 (177 $\mathrm{mg}, 0.239 \mathrm{mmol}, 0.1$ equiv) were added to an argon purged flame dried flask. After purging with argon, silver hexafluoroantimonate (very hygroscopic!) $(85.5 \mathrm{mg}, 0.249 \mathrm{mmol}, 0.11$ equiv) was added quickly followed again by an argon purge. Toluene $(2.2 \mathrm{~mL})$ was added and the reaction was wrapped in aluminum foil and stirred at room temperature for $25 \mathrm{~h}$ in a closed environment. Toluene $(0.5 \mathrm{~mL})$ was added at the four-hour point to keep the reaction stirring, because the product was not very soluble and the reaction mixture solidified. The reaction mixture was directly loaded onto a short silica gel column and all compounds were eluted using ethyl acetate and $2 / 1$ chloroform/methanol. The crude material was purified using silica gel chromatography. (S)-2,2'-Bis(3,5-di-tert-butyl-2hydorxybenzilideneamino)-1,1'-binaphthyl was eluted using 9/1 hexanes/ethyl acetate, followed by 3-acetoxy-4-benzyloxybenzldehyde which eluted with 4/1 hexanes/ethyl acetate. Trace amounts of the trans compound (4S,5R)-5-(3-acetoxy-4benzyloxyphenyl)-2-(4-methoxyphenyl)-4,5-dihydrooxazole-4-(carboxylic acid methyl ester) 10 eluted with $1 / 1$ hexanes/ethyl acetate and $1 / 2$ to $1 / 4$ hexanes/ethyl acetate eluted the product 9 (1.11 g, $2.34 \mathrm{mmol}, 99 \%)$. Enantioselectivity was determined to be $96 \%$ ee using Chiralpak AS column eluting with $15 \%$ ethanol in hexanes. The major isomer eluted at $16.07 \mathrm{~min}$ and the minor isomer eluted at $25.95 \mathrm{~min} . \mathrm{R}_{\mathrm{f}}=0.2(1 / 1$ hexanes/ethyl acetate); IR (film) $1756 \mathrm{~m}, 1645 \mathrm{~m}, 1609 \mathrm{~m}, 1514 \mathrm{~s}, 1455 \mathrm{w}, 1346 \mathrm{w}, 1303 \mathrm{w}, 1259 \mathrm{~s}$, 1200 s, 1171 s, 1122 m, 1084 m, 1026 m, 910 w, 840 w, 743 w, 693 w; ${ }^{1}$ H NMR (500

\footnotetext{
${ }^{2}$ Haensel, R.; Su, T-L.; Schulz, J. Chem. Ber. 1977, 110, 3664.

${ }^{3}$ Evans, D. A.; Janey, J. M.; Magomedov, N.; Tedrow, J. S. Angew. Chem. Int. Ed. 2001, 40, 1884-1888.
} 
$\mathrm{MHz}, \mathrm{CDCl}_{3}$ ) $\delta 8.00$ (dd, J=2, $7 \mathrm{~Hz}, 2 \mathrm{H}$ ), 7.34-7.28 (mult, 5H), 7.06 (dd, J=2, $8 \mathrm{~Hz}, 1 \mathrm{H}$ ), $6.98(\mathrm{~d}, \mathrm{~J}=2 \mathrm{~Hz}, 1 \mathrm{H}), 6.93(\mathrm{dd}, \mathrm{J}=2,7 \mathrm{~Hz}, 2 \mathrm{H}), 6.91(\mathrm{~d}, \mathrm{~J}=8 \mathrm{~Hz}, 1 \mathrm{H}), 5.80(\mathrm{~d}, \mathrm{~J}=11 \mathrm{~Hz}$, $1 \mathrm{H}), 5.20(\mathrm{~d}, \mathrm{~J}=11 \mathrm{~Hz}, 1 \mathrm{H}), 5.06(\mathrm{~s}, 2 \mathrm{H}), 3.84(\mathrm{~s}, 3 \mathrm{H}), 3.26(\mathrm{~s}, 3 \mathrm{H}), 2.22(\mathrm{~s}, 3 \mathrm{H}) ;{ }^{13} \mathrm{C}$ NMR $\left(125.5 \mathrm{MHz}, \mathrm{CDCl}_{3}\right) \delta 164.9,168.7,166.2,162.6,150.2,140.0,136.3,130.5$, 128.9, 128.5, 127.9, 127.0, 124.8, 120.9, 119.1, 113.8, 113.4, 81.9, 74.0, 70.5, 55.3, 51.9, 20.5; HRMS calculated for $\mathrm{C}_{27} \mathrm{H}_{25} \mathrm{NO}_{7}(\mathrm{M}+\mathrm{H}): 476.1704$, found (MALDI): 476.1705 .

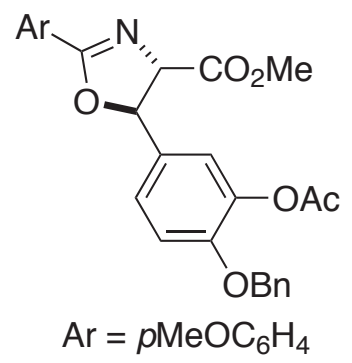

(4S,5R)-5-(3-Acetoxy-4-benzyloxyphenyl)-2-(4-methoxyphenyl)-4,5-dihydrooxazole-4-(carboxylic acid methyl ester) (10). cis-Oxazoline 9 (688 $\mathrm{mg}, 1.45 \mathrm{mmol}, 1$ equiv) was dissolved in dichloromethane $(10 \mathrm{~mL})$ at ambient temperature. 1,8-Diazabicyclo[5.4.0]undec-7-ene $(43 \mu \mathrm{L}, 0.29 \mathrm{mmol}, 0.2$ equiv) was added and the reaction mixture stirred for $2.5 \mathrm{~h}$. The reaction was diluted with dichloromethane and then neutralized using $0.5 \mathrm{~N}$-hydrochloric acid. The layers were separated and the aqueous layer was extracted twice more with dichloromethane. The combined organic layers were dried over anhydrous sodium sulfate. Solvent was removed under reduced pressure and the crude material was purified on silica gel chromatography. transOxazoline 10 was eluted using $3 / 1$ to $2 / 1$ hexanes/ethyl acetate $(599 \mathrm{mg}, 1.26 \mathrm{mmol}$, $87 \%)$, followed by recovered $9(50.4 \mathrm{mg}, 0.106 \mathrm{mmol}, 7 \%)$ which eluted with $1 / 1$ hexanes/ethyl acetate. $\mathrm{R}_{\mathrm{f}}=0.3\left(1 / 1\right.$ hexanes/ethyl acetate); ${ }^{1} \mathbf{H} \mathbf{N M R}\left(400 \mathrm{MHz}, \mathrm{CDCl}_{3}\right) \delta$ $8.00(\mathrm{~d}, \mathrm{~J}=8 \mathrm{~Hz}, 2 \mathrm{H}$ ), 7.47-7.62 (mult, 5H), 7.20 (d, J=8 Hz, 1H), 7.1- (s, $1 \mathrm{H}), 7.00$ (d, $\mathrm{J}=8 \mathrm{~Hz}, 1 \mathrm{H}), 6.93(\mathrm{~d}, \mathrm{~J}=8 \mathrm{~Hz}, 2 \mathrm{H}), 5.81(\mathrm{~d}, \mathrm{~J}=8 \mathrm{~Hz}, 1 \mathrm{H}), 5.11(\mathrm{~s}, 2 \mathrm{H}), 4.78(\mathrm{~d}, \mathrm{~J}=8 \mathrm{~Hz}$, $1 \mathrm{H}), 3.88(\mathrm{~s}, 3 \mathrm{H}), 3.85$ (s, 3H), 2.27 (s, 3H).

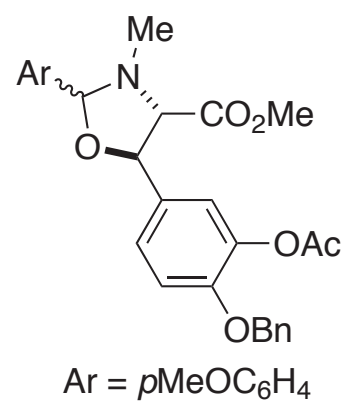

(4S,5R)-5-(3-Acetoxy-4-benzyloxyphenyl)-2-(4-methoxyphenyl)-3-methyl-oxazolidine-4-carboxylic acid methyl ester (11). Toluene-azeotroped 10 (54.4 mg, $0.114 \mathrm{mmol}, 1$ equiv) was dissolved in dichloromethane $(1 \mathrm{~mL})$ at ambient temperature. Freshly distilled (from calcium hydride) methyl trifluoromethanesulfonate ( $15 \mathrm{~mL}, 0.13 \mathrm{mmol}, 1.2$ equiv) was added and the reaction was stirred $2 \mathrm{~h}$. Toluene was added and all solvent was removed under reduced pressure. The material was resuspended in dichloromethane $(1 \mathrm{~mL})$ at $0{ }^{\circ} \mathrm{C}$. Saturated sodium bicarbonate solution $(1 \mathrm{~mL})$ and sodium borohydride $(9.3 \mathrm{mg}, 0.25 \mathrm{mmol}, 2$ equiv) were added all at once with vigorous stirring. Bubbling ensued and the reaction was stirred $15 \mathrm{~min}$ before being diluted with dichloromethane and water. The layers were separated and the aqueous layer was extracted twice more with dichloromethane. The combined organic layers were dried over anhydrous $\mathrm{Na}_{2} \mathrm{SO}_{4}$. Solvent was removed under reduced pressure and the crude material was purified using silica gel chromatography. The desired $\mathbf{1 1}$ and the over-reduced 3-(3-acetoxy-4benzyloxyphenyl)-3-hydroxy-2-benzylmethylaminopropionic acid methyl ester 14 eluted together using $4 / 1$ hexanes/ethyl acetate and $48.3 \mathrm{mg}$ of the mixture was obtained and the yields were $62 \%$ and $24 \%$ respectively, as determined by the integration of ${ }^{1} \mathrm{H}$ NMR spectrum. Recovered $10(3.5 \mathrm{mg}, 0.0074 \mathrm{mmol}, 6 \%)$ was obtained by eluting with $2 / 1$ to $1 / 2$ hexanes/ethyl acetate. 
5-(3-Acetoxy-4-benzyloxyphenyl)-1-(4-methoxyphenyl)-3-methyloxazolidine-4carboxylic acid methyl ester 11. $\mathrm{R}_{\mathrm{f}}=0.8\left(1 / 1\right.$ hexanes/ethyl acetate); ${ }^{1} \mathbf{H}$ NMR $(500 \mathrm{MHz}$, $\mathrm{CDCl}_{3}$ ) $\delta 7.49$ (d, J=8 Hz, 2H), 7.40-6.91 (mult, 8H), 6.90 (d, J=8 Hz, 2H), 5.32 (d, J=7 $\mathrm{Hz}, 1 \mathrm{H}), 5.07(\mathrm{~s}, 2 \mathrm{H}), 5.00(\mathrm{~s}, 1 \mathrm{H}), 3.80(\mathrm{~s}, 3 \mathrm{H}), 3.77(\mathrm{~s}, 3 \mathrm{H}), 3.38(\mathrm{~d}, \mathrm{~J}=7 \mathrm{~Hz}, 1 \mathrm{H}), 2.31$ (s, 3H), $2.26(\mathrm{~s}, 3 \mathrm{H})$.

3-(3-Acetoxy-4-benzyloxyphenyl)-3-hydroxy-2-benzylmethylaminopropionic acid methyl ester 14. $\mathrm{R}_{\mathrm{f}}=0.8$ (1/1 hexanes/ethyl acetate); ${ }^{1} \mathbf{H} \mathbf{~ N M R}\left(500 \mathrm{MHz}, \mathrm{CDCl}_{3}\right) \delta$ 7.387.27 (mult, 5H), 7.22 (d, J=8 Hz, 2H), 7.12 (dd, J=2, $8 \mathrm{~Hz}, 1 \mathrm{H}), 7.01$, (d, J=2 Hz, 1H), $6.90(\mathrm{~d}, \mathrm{~J}=8 \mathrm{~Hz}, 1 \mathrm{H}), 6.87(\mathrm{~d}, \mathrm{~J}=8 \mathrm{~Hz}, 2 \mathrm{H}), 5.04(\mathrm{~s}, 2 \mathrm{H}), 4.80(\mathrm{~d}, \mathrm{~J}=10 \mathrm{~Hz}, 1 \mathrm{H}), 4.29$ (br, $1 \mathrm{H}), 3.80(\mathrm{~s}, 3 \mathrm{H}), 3.79(\mathrm{~d}, \mathrm{~J}=10 \mathrm{~Hz}, 1 \mathrm{H}), 3.59(\mathrm{~s}, 3 \mathrm{H}), 3.55(\mathrm{~d}, \mathrm{~J}=13 \mathrm{~Hz}, 1 \mathrm{H}), 3.25(\mathrm{~d}$, $\mathrm{J}=10 \mathrm{~Hz}, 1 \mathrm{H}), 2.32(\mathrm{~s}, 3 \mathrm{H}), 2.23$ (s, 3H).

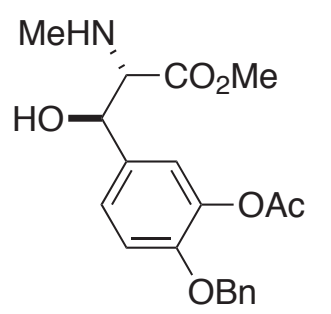

(4S,5R)-3-(3-Acetoxy-4-benzyloxyphenyl)-3-hydroxy-2methylaminopropionic acid methyl ester (12). Oxazolidine 11 and the over-reduced $14(133 \mathrm{mg}, 0.271 \mathrm{mmol}, 1$ equiv) were dissolved in THF $(10 \mathrm{~mL})$ at $0{ }^{\circ} \mathrm{C}$ and $0.1 \mathrm{~N}$ oxalic acid solution $(10$ $\mathrm{mL}$ ) was added. The reaction mixture was stirred overnight at $4{ }^{\circ} \mathrm{C}$. THF was removed under reduced pressure and the reaction mixture was diluted with ethyl acetate. Saturated sodium bicarbonate solution was added until the $\mathrm{pH}$ of the solution was 8 . The layers were separated and the aqueous layer was extracted twice more with ethyl acetate. The combined organic layers were washed with brine and dried over anhydrous $\mathrm{Na}_{2} \mathrm{SO}_{4}$. The solvent was removed under reduced pressure and the crude material was purified using silica gel column chromatography. 4-Methoxybenzaldehyde and the over reduced byproduct 14 eluted with $4 / 1$ hexanes/ethyl acetate. The desired product $12(81.2 \mathrm{mg}, 0.217 \mathrm{mmol}, 80 \%)$ eluted with $100 \%$ ethyl acetate and $10 / 1$ ethyl acetate/methanol. $\mathrm{R}_{\mathrm{f}}=0.3$ (ethyl acetate); ${ }^{1} \mathbf{H}$ NMR $\left(500 \mathrm{MHz}, \mathrm{CDCl}_{3}\right.$ ) $\delta$ 7.40-7.26 (mult, 5H), 7.14 (dd, J=2, $\left.8 \mathrm{~Hz}, 1 \mathrm{H}\right), 7.03$ (d, $\mathrm{J}=2 \mathrm{~Hz}, 1 \mathrm{H}), 6.59(\mathrm{~d}, \mathrm{~J}=8 \mathrm{~Hz}, 1 \mathrm{H}), 5.07(\mathrm{~s}, 2 \mathrm{H}), 4.51(\mathrm{~d}, \mathrm{~J}=8 \mathrm{~Hz}, 1 \mathrm{H}), 3.55(\mathrm{~s}, 3 \mathrm{H}), 3.16$ $(\mathrm{d}, \mathrm{J}=8 \mathrm{~Hz}, 1 \mathrm{H}), 2.37$ (s, 3H), 2.24 (s, 3H).

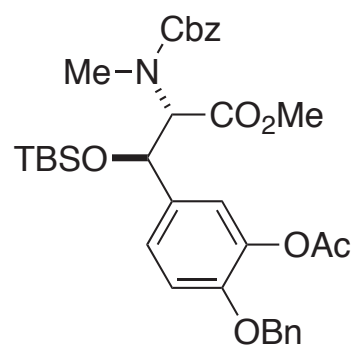

(4S,5R)-3-(3-Acetoxy-4-benzyloxyphenyl)-2-
(benzyloxycarbonylmethylamino)-3-tert-butyldimethylsilyl-
oxypropionic acid methyl ester (13). Amino alcohol 12 (77.8 $\mathrm{mg}, 0.208 \mathrm{mmol}, 1$ equiv) was dissolved in acetonitrile $(2 \mathrm{~mL})$ at ambient temperature. Sodium bicarbonate $(91.0 \mathrm{mg}, 1.08 \mathrm{mmol}$, 5 equiv) was added followed by addition of benzyl chloroformate ( $45 \mu \mathrm{L}, 0.32 \mathrm{mmol}, 1.5$ equiv). The reaction was stirred for $4 \mathrm{~h}$ and then diluted with ethyl acetate. Water was added and the layers were separated. The aqueous layer was extracted twice more with ethyl acetate. The combined organic layers were washed with brine and dried over anhydrous sodium sulfate. The solvent was removed under reduced pressure and the crude material was purified using silica gel column chromatography. The product was obtained by eluting with a gradient of 9/1 to 2/1 hexanes/ethyl acetate. 3-(3-Acetoxy-4-benzyloxyphenyl)-2(benzyloxycarbonylmethylamino)-3-hydroxypropionic acid methyl ester (97.8 $\mathrm{mg}, 0.193$ mmol, 93\%) was obtained. $\mathrm{R}_{\mathrm{f}}=0.4\left(1 / 1\right.$ hexanes/ethyl acetate); ${ }^{1} \mathbf{H} \mathbf{~ N M R}(500 \mathrm{MHz}$, DMSO- $\mathrm{d}_{6}, 22{ }^{\circ} \mathrm{C}$ ) $\delta$ 7.39-7.02 (mult, 5H), 5.95 (br, 1H), 5.22-4.85 (mult, 6H), 3.56 (3.59) 
(s, 3H), 2.96 (2.92) (s, 3H), 2.23 (2.22) (s, 3H); ${ }^{1} \mathbf{H}$ NMR (500 MHz, DMSO-d $\left.6,70{ }^{\circ} \mathrm{C}\right)$ ઈ 7.40-7.08 (mult, 5H), 7.52 (br, 1H), 5.19-4.84 (mult, 6H), 3.56 (s, 3H), 2.97 (s, 3H), 2.20 (s, 3H); MS calculated for $\mathrm{C}_{28} \mathrm{H}_{29} \mathrm{O}_{8} \mathrm{~N}(\mathrm{M}+\mathrm{Na})$ : 530, found (ESI): 530. The chemical shifts in parentheses are the shifts for the minor rotamer.

3-(3-Acetoxy-4-benzyloxyphenyl)-2-(benzyloxycarbonylmethylamino)-3-

hydroxypropionic acid methyl ester $(97.8 \mathrm{mg}, 0.193 \mathrm{mmol}, 1$ equiv) was dissolved in pyridine $(1 \mathrm{~mL})$ at ambient temperature. tert-Butyldimethylsilyl chloride $(182 \mathrm{mg}, 1.21$ mmol, 6 equiv) and silver nitrate (202 mg, 1.19 mmol, 6 equiv) were added and the reaction mixture was stirred for $17 \mathrm{~h}$. Pyridine was removed under reduced pressure and the reaction mixture was directly loaded onto a silica gel column. Elution with a gradient of 19/1 to 6/1 hexanes/ethyl acetate afforded $13(109 \mathrm{mg}, 0.175 \mathrm{mmol}, 91 \%)$. $\mathrm{R}_{\mathrm{f}}=0.6(1 / 1$ hexanes/ethyl acetate) ${ }^{1} \mathbf{H}$ NMR (500 MHz, DMSO-d ${ }_{6}$ ) $\delta$ 7.38-6.85 (mult, $\left.13 \mathrm{H}\right), 5.34-$ 4.77 (mult, 6H), 3.66 (3.63) (s, 3H), 3.10 (3.12) (s, 3H), 2.23 (2.24) (s, 3H), 0.84 (0.83) $(\mathrm{s}, 9 \mathrm{H}), 0.01(-0.01)(\mathrm{s}, 3 \mathrm{H}),-0.23(-0.24)(\mathrm{s}, 3 \mathrm{H})$. The chemical shifts in parentheses are the shifts for the minor rotamer.

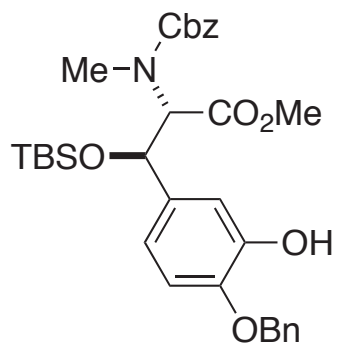

(4S,5R)-2-(Benzyloxycarbonylamino)-3-(3-benzyloxy-4-
hydroxyphenyl)-3-(tert-butyldimethylsilyloxy)propionic acid methyl ester (4). Fully protected 13 (109 $\mathrm{mg}, 0.175 \mathrm{mmol}, 1$ equiv) was dissolved in THF $(3 \mathrm{~mL})$ and methanol $(0.5 \mathrm{~mL})$ at 0 ${ }^{\circ} \mathrm{C}$. Potassium tert-butoxide $(1 \mathrm{M}$ solution in THF) $(265 \mu \mathrm{L}$, $0.265 \mathrm{mmol}, 1.5$ equiv) was added and the reaction was stirred for 10 min. The reaction was diluted with ethyl acetate and neutralized by adding $\mathrm{pH} 2.5$ phosphate buffer $(2 \mathrm{~mL})$. The layers

were separated. The aqueous layer was extracted twice more with ethyl acetate. The combined organic layers were washed with brine and dried over anhydrous sodium sulfate. The solvent was removed under reduced pressure and the crude material was purified using silica gel column chromatography. The product was obtained by eluting with $5 / 1$ hexanes/ethyl acetate giving $4(96.1 \mathrm{mg}, 0.166 \mathrm{mmol}, 95 \%) . \quad \mathrm{R}_{\mathrm{r}}=0.5(1 / 1$ hexanes/ethyl acetate); ${ }^{1} \mathbf{H}$ NMR (500 MHz, DMSO-d $\left.{ }_{6}\right) \delta$ 7.47-6.66 (mult, $\left.13 \mathrm{H}\right), 5.22-$ 4.79 (mult, 6H), 3.60 (3.58) (s, 3H), 3.06 (3.00) (s, 3H), 0.80 (s, 9H), -0.02 (-0.03) (s, $3 \mathrm{H}),-0.28(-0.27)(\mathrm{s}, 3 \mathrm{H})$. The chemical shifts in parentheses are the shifts for the minor rotamer.

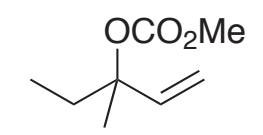

Carbonic acid-1-ethyl-1-methylallyl ester methyl ester (16). 3Methylpent-1-ene-3-ol (1.97 mL, $16.5 \mathrm{mmol}, 1$ equiv) was dissolved in THF $(30 \mathrm{~mL})$ at $0{ }^{\circ} \mathrm{C}$. Butyl lithium $(1.6 \mathrm{M}$ solution in hexanes) $(11.5$ $\mathrm{mL}, 18.4 \mathrm{mmol}, 1.1$ equiv) was added dropwise and the reaction mixture was stirred for $30 \mathrm{~min}$. Methyl chloroformate $(1.9 \mathrm{~mL}, 25 \mathrm{mmol}, 1.5$ equiv) was added dropwise and the reaction was gradually warmed to room temperature and stirred at room temperature for 4 $\mathrm{h}$. The reaction was acidified by the addition of $1 \mathrm{~N}$ hydrochloric acid, and the organic products were extracted three times with ether. The combined organic layers were dried over anhydrous $\mathrm{MgSO}_{4}$. Solvents were carefully removed under reduced pressure, and the product was distilled under reduced pressure. Product 16 distilled at $58{ }^{\circ} \mathrm{C}, 5 \mathrm{~mm} \mathrm{Hg}$ $(2.19 \mathrm{~g}, 13.9 \mathrm{mmol}, 84 \%) . \mathrm{R}_{\mathrm{f}}=0.8$ (5/1 hexanes/ethyl acetate); IR (film) $2976 \mathrm{w}, 1752 \mathrm{~m}$, 1442 w, 1275 s, 1134 w, 993 w, 943 w, 871 w, 794 w; ${ }^{1} \mathbf{H}$ NMR $\left(400 \mathrm{MHz}, \mathrm{CDCl}_{3}\right) \delta$ 
5.99 (dd, J=12, $20 \mathrm{~Hz}, 1 \mathrm{H}), 5.22(\mathrm{~d}, \mathrm{~J}=12 \mathrm{~Hz}, 1 \mathrm{H}), 5.18$ (d, J=20 Hz, 1H), $3.72(\mathrm{~s}, 3 \mathrm{H})$, $1.86(\mathrm{q}, \mathrm{J}=8 \mathrm{~Hz}, 2 \mathrm{H}), 1.57(\mathrm{~s}, 3 \mathrm{H}), 0.91(\mathrm{t}, \mathrm{J}=8 \mathrm{~Hz}, 3 \mathrm{H}) ;{ }^{13} \mathbf{C} \mathbf{N M R}\left(125.5 \mathrm{MHz}, \mathrm{CDCl}_{3}\right) \delta$ 153.7, 140.6, 114.0, 84.6, 53.8, 32.3, 22.2, 7.7;

Carbonic acid methyl ester-3-methylpent-2-enyl ester (17).

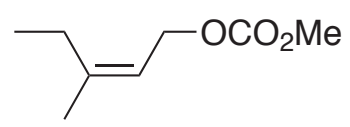

3-Methylpent-2-en-1-ol ${ }^{4}$ (1.49 g, 14.9 mmol, 1 equiv) was diluted with dichloromethane $\left(\begin{array}{lllll}50 & \mathrm{~mL}\end{array}\right)$ at $0{ }^{\circ} \mathrm{C}$. 4Dimethylaminopyridine ( $2.55 \mathrm{~g}, 20.8 \mathrm{mmol}, 1.4$ equiv) was added followed by methyl chloroformate $(1.38 \mathrm{~mL}, 17.9 \mathrm{mmol}, 1.2$ equiv). The reaction was allowed to warm to room temperature and stirred $10 \mathrm{~h}$. $1 \mathrm{~N} \mathrm{HCl}$ solution $(100 \mathrm{~mL})$ was added and the layers were separated. The aqueous layer was then extracted with dichloromethane $(3 \times 50 \mathrm{~mL})$. The combined organic layers were dried over anhydrous $\mathrm{MgSO}_{4}$. After careful removal of the solvent under reduced pressure, 17 was distilled under reduced pressure $\left(104{ }^{\circ} \mathrm{C}, 30 \mathrm{mmHg}\right)(1.84 \mathrm{~g}, 11.6 \mathrm{mmol}, 78 \%) . \quad \mathrm{R}_{\mathrm{f}}=0.8(5 / 1$ hexanes/ethyl acetate); IR (film) 2972 s, 1801 s, 1750 s, 1445 s, 1381 m, 1270 s, 1089 m, 949 m, 793 s; ${ }^{1} \mathbf{H}$ NMR (400 MHz, CDCl $)$ \& 5.34 (t, J=10 Hz, 1H), 4.64 (d, J=10 Hz, 2H), $3.76(\mathrm{~s}, 3 \mathrm{H}), 2.14$ (q, J=8 Hz, 2H), 1.01 (t, J=8 Hz, 3H); ${ }^{13} \mathbf{C}$ NMR $(125.5 \mathrm{MHz}$, $\left.\mathrm{CDCl}_{3}\right) \delta$ 155.7, 145.4, 117.3, 64.1, 54.5, 24.9, 22.7, 12.8;

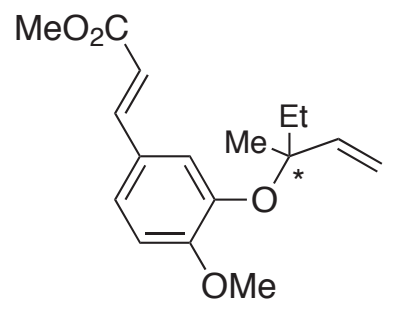
temperature. $16(77.3 \mathrm{mg}, 0.489 \mathrm{mmol}, 2$ equiv) was added as a solution in dichloromethane $(1.5 \mathrm{~mL})$. The reaction was complete within $30 \mathrm{~min}$ and the reaction mixture was loaded onto a silica gel column. 21 (68.1 mg, $0.235 \mathrm{mmol}, 97 \%, 25 \%$ ee $)$ was obtained upon elution with $15 / 1$ to $3 / 1$ hexanes/ethyl acetate. $\mathrm{R}_{\mathrm{f}}=0.7(2 / 1$ hexanes/ethyl acetate); $\mathrm{Rt}=16.0 \mathrm{~min}$ (major isomer), $17.5 \mathrm{~min}$ (minor isomer), Chiralpak $\mathrm{AD}\left(1 \mathrm{~mL} / \mathrm{min}, 1 \%\right.$ isopropyl alcohol/heptane); ${ }^{1} \mathbf{H}$ NMR $\left(400 \mathrm{MHz}, \mathrm{CDCl}_{3}\right) \delta 7.58$ (d, $\mathrm{J}=16 \mathrm{~Hz}, 1 \mathrm{H}), 7.23(\mathrm{~s}, 1 \mathrm{H}), 7.15(\mathrm{~d}, \mathrm{~J}=12 \mathrm{~Hz}, 1 \mathrm{H}), 6.86(\mathrm{~d}, \mathrm{~J}=12 \mathrm{~Hz}, 1 \mathrm{H}), 6.21(\mathrm{~d}, \mathrm{~J}=16$ $\mathrm{Hz}, 1 \mathrm{H}), 6.07(\mathrm{dd}, \mathrm{J}=12,20 \mathrm{~Hz}, 1 \mathrm{H}), 5.20(\mathrm{~d}, \mathrm{~J}=12 \mathrm{~Hz}, 1 \mathrm{H}), 5.14(\mathrm{~d}, \mathrm{~J}=20 \mathrm{~Hz}, 1 \mathrm{H}), 3.84$ (s, 3H), 3.80 (s, 3H), 1.93-1.76 (mult, 2H), 1.38 (s, 3H), 1.00 (t, J=8 Hz, 3H).

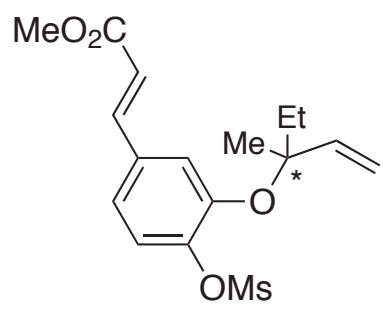

3-[3-(1-Ethyl-1-methylallyloxy)-4-methanesulfonyloxyphenyl]acrylic acid methyl ester (23). This compound was synthesized in a similar manner to $\mathbf{2 1}$ starting from $\mathbf{2 2}$ (30.1 $\mathrm{mg}, 0.111 \mathrm{mmol}, 1$ equiv). Adduct 23 (11.6 $\mathrm{mg}, 0.0327 \mathrm{mmol}$, $29 \%, 10 \% e e$ ) was obtained upon elution with $10 / 1$ to $2 / 1$ hexanes/ethyl acetate followed by recovered starting material

4 Sen, S. E.; Ewing, G. J. J. Org. Chem. 1997, 62, 3529-3536.

${ }^{5}$ Trost, B. M.; Van Vranken, D. L.; Bingel, C. J. Am. Chem. Soc. 1992, 114, 9327-9343.

${ }^{6}$ Ukai, T.; Kawazura, H.; Ishii, Y.; Bonnet, J. J.; Ibers, J. A. J. Organomet. Chem. 1974, 65, $253-266$. 
23 (21.5 mg, $0.0790 \mathrm{mmol}, 71 \%) . \quad \mathrm{R}_{\mathrm{f}}=0.5$ (2/1 hexanes/ethyl acetate); $\mathrm{Rt}=10.9 \mathrm{~min}$ (minor isomer), $11.3 \mathrm{~min}$ (major isomer), Chiralpak $\mathrm{AD}(1 \mathrm{~mL} / \mathrm{min}, 10 \%$ isopropyl alcohol/heptane); ${ }^{1} \mathbf{H}$ NMR (400 MHz, $\mathrm{CDCl}_{3}$ ) $\delta 7.57$ (d, J=16 Hz, 1H), 7.36-7.29 (mult, 2H), $7.16(\mathrm{~d}, \mathrm{~J}=12 \mathrm{~Hz}, 1 \mathrm{H}), 6.30$ (d, J=12 Hz, 1H), 6.07 (dd, J=12, $20 \mathrm{~Hz}, 1 \mathrm{H}), 5.29$ (d, $\mathrm{J}=12 \mathrm{~Hz}, 1 \mathrm{H}), 5.21(\mathrm{~d}, \mathrm{~J}=20 \mathrm{~Hz}, 1 \mathrm{H}), 3.79$ (s, 3H), 3.20 (s, 3H), 1.93-1.80 (mult, 2H), 1.45 (s, 3H), 0.99 (t, J=8 Hz, 3H).

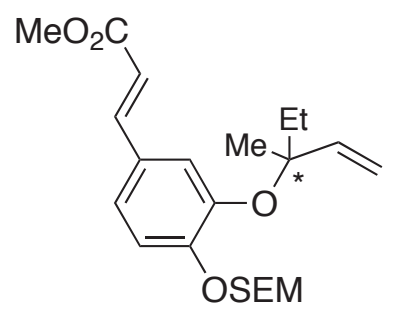

3-\{3-(1-Ethyl-1-methylallyloxy)-4-[2-(trimethylsilyl)ethoxymethoxy]phenyl\}acrylic acid methyl ester (25). This compound was synthesized in a similar manner to $\mathbf{2 1}$ starting from 24 (72.1 mg, $0.222 \mathrm{mmol}, 1$ equiv). Adduct 25 (78.9 mg, $0.194 \mathrm{mmol}, 87 \%$, 18\%ee) was obtained upon elution with $20 / 1$ to $4 / 1$ hexanes/ethyl acetate followed by recovered starting material 24 (2.9 mg, $0.0894 \mathrm{mmol}, 4 \%) . \quad \mathrm{R}_{\mathrm{f}}=0.7 \quad(2 / 1$

hexanes/ethyl acetate); $\mathrm{Rt}=8.7 \mathrm{~min}$ (major isomer), $9.2 \mathrm{~min}$ (minor isomer), Chiralpak AD (1 mL/min, 1\% isopropyl alcohol/heptane); ${ }^{1} \mathbf{H} \mathbf{N M R}\left(400 \mathrm{MHz}, \mathrm{CDCl}_{3}\right) \delta 7.57$ (d, $\mathrm{J}=16 \mathrm{~Hz}, 1 \mathrm{H}), 7.24$ (d, J=2 Hz, 1H), 7.12 (d, J=2 Hz, 1H), 7.11 (s, 1H), 6.23 (d, J=16 Hz, $1 \mathrm{H}), 6.08(\mathrm{dd}, \mathrm{J}=16,20 \mathrm{~Hz}, 1 \mathrm{H}), 5.24(\mathrm{~s}, 2 \mathrm{H}), 5.20(\mathrm{~d}, \mathrm{~J}=12 \mathrm{~Hz}, 1 \mathrm{H}), 5.15$ (d, J=20 Hz, $1 \mathrm{H}), 3.79$ (s, 3H), $3.76(\mathrm{~d}, \mathrm{~J}=8 \mathrm{~Hz}, 1 \mathrm{H}), 3.74$ (d, J=12 Hz, 1H), 1.91-1.74 (mult, 2H), 1.36 $(\mathrm{s}, 3 \mathrm{H}), 0.98$ (t, J=8 Hz, 3H), 0.96 (dd, J=8, $8 \mathrm{~Hz}, 2 \mathrm{H}), 0.00$ (s, 9H).

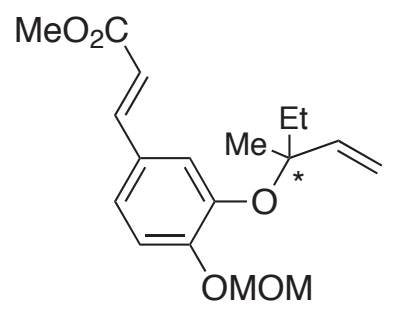

3-[3-(1-Ethyl-1-methylallyloxy)-4-methoxymethoxyphenyl]acrylic acid methyl ester (27). Using 16: This compound was synthesized in a similar manner to $\mathbf{2 1}$ starting from 26 (53.2 mg, $0.223 \mathrm{mmol}, 1$ equiv). Adduct 27 (67.5 mg, $0.211 \mathrm{mmol}, 94 \%, 20 \% e e$ ) was obtained upon elution with $20 / 1$ to $3 / 1$ hexanes/ethyl acetate.

Using 17: Phenol 26 (52.3 mg, $0.223 \mathrm{mmol}, 1$ equiv), (1S,2S)1,2-bis(2-diphenylphosphinobenzoyl)aminocyclohexane $19(7.7 \mathrm{mg}, 0.011 \mathrm{mmol}, 0.05$ equiv) and tris(dibenzylideneacetone)dipalladium(0)-chloroform (2.3 $\mathrm{mg}, 0.0022 \mathrm{mmol}$, 0.01 equiv) were dissolved in dichloromethane (degassed by bubbling argon for $20 \mathrm{~min}$ ) $(0.5 \mathrm{~mL})$ at room temperature. Carbonate 16 ( $70.6 \mathrm{mg}, 0.446 \mathrm{mmol}, 2$ equiv) was added as a solution in dichloromethane $(1.5 \mathrm{~mL})$. The reaction was complete within $30 \mathrm{~min}$ and the reaction mixture was loaded onto a silica gel column. Adduct 27 (31.4 mg, 0.0981 mmol, 44\%, 82\%ee) was obtained upon elution with $20 / 1$ to $3 / 1$ hexanes/ethyl acetate followed by recovered starting material $26(28.7 \mathrm{mg}, 0.120 \mathrm{mmol}, 54 \%) . \quad \mathrm{R}_{\mathrm{f}}=0.6(2 / 1$ hexanes/ethyl acetate); $\mathrm{Rt}=29.3 \mathrm{~min}$ (minor isomer), $31.6 \mathrm{~min}$ (major isomer), Chiralcel OJ (0.5 mL/min, $0.8 \%$ isopropyl alcohol/heptane); $\mathrm{Rt}=16.8 \mathrm{~min}$ (major isomer), $18.4 \mathrm{~min}$ (minor isomer), Chiralpak AD $(0.9 \mathrm{~mL} / \mathrm{min}, 1 \%$ isopropyl alcohol/heptane $) ;{ }^{1} \mathbf{H}$ NMR $\left(400 \mathrm{MHz}, \mathrm{CDCl}_{3}\right) \delta 7.57$ (d, J=16 Hz, 1H), 7.23 (s, 1H), 7.11-7.04 (mult, 2H), 6.23 (d, $\mathrm{J}=16 \mathrm{~Hz}, 1 \mathrm{H}), 6.07$ (dd, J=12, $20 \mathrm{~Hz}, 1 \mathrm{H}), 5.20(\mathrm{~d}, \mathrm{~J}=12 \mathrm{~Hz}, 1 \mathrm{H}), 5.15(\mathrm{~d}, \mathrm{~J}=20 \mathrm{~Hz}, 1 \mathrm{H})$, 3.79 (s, 3H), 3.52 (s, 3H), 1.92-1.75 (mult, 2H), 1.37 (s, 3H), 0.99 (t, J=8 Hz, 3H). 


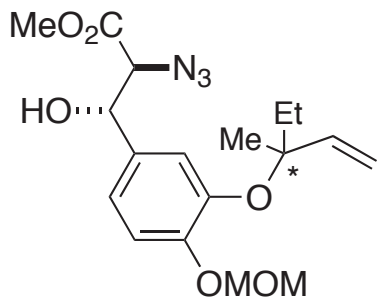

(2S,3S)-2-Azido-3-[3-(1-ethyl-1-methylallyloxy)-4methoxymethoxyphenyl]-3-hydroxypropionic acid methyl ester (29). Using 16: This compound was synthesized in a similar manner to $\mathbf{2 1}$ starting from $\mathbf{2 8}(16.2 \mathrm{mg}, 0.0545 \mathrm{mmol}$, 1 equiv). Adduct 29 ( $17.5 \mathrm{mg}, 0.0461 \mathrm{mmol}, 85 \%, 22 \%$ ee $)$ was obtained upon elution with $10 / 1$ to $1 / 1$ hexanes/ethyl acetate followed by recovered starting material $28(0.5 \mathrm{mg}$, $0.0017 \mathrm{mmol}, 3 \%$ ).

Using 17: This compound was synthesized in a similar manner to $\mathbf{2 7}$ starting from $\mathbf{2 8}$ (16.2 $\mathrm{mg}, 0.0545 \mathrm{mmol}, 1$ equiv) and 17. Adduct 29 (2.28 mg, $0.0060 \mathrm{mmol}, 11 \%$, $80 \% e e$ ) was obtained upon elution with $10 / 1$ to $1 / 1$ hexanes/ethyl acetate followed by recovered starting material 28 (12.0 mg, $0.0403 \mathrm{mmol}, 74 \%) . \mathrm{R}_{\mathrm{f}}=0.7$ (1/1 hexanes/ethyl acetate); $\mathrm{Rt}=12.5 \mathrm{~min}$ (major isomer), $13.0 \mathrm{~min}$ (minor isomer), Chiralpak $\mathrm{AD}$ (0.9 $\mathrm{mL} / \mathrm{min}, 10 \%$ isopropyl alcohol/heptane); ${ }^{1} \mathbf{H}$ NMR $\left(400 \mathrm{MHz}, \mathrm{CDCl}_{3}\right) \delta$ 7.96-6.92 (mult, 3H), 6.13-6.03 (mult, 1H), 5.18 (s, 2H), 5.22-5.10 (mult, 1H), 4.90 (d, J=8 Hz, $1 \mathrm{H}), 4.02(\mathrm{~d}, \mathrm{~J}=8 \mathrm{~Hz}, 1 \mathrm{H}), 3.80$ (s, 3H), 3.56 (s, 3H), 1.91-1.73 (mult, 2H), 1.38 (s, 3H), $0.99(\mathrm{t}, \mathrm{J}=8 \mathrm{~Hz}, 3 \mathrm{H})$.

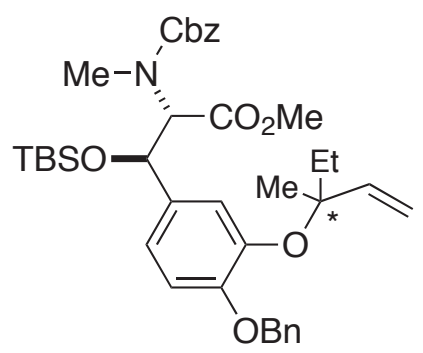

General Procedure for: 2-(Benzyloxycarbonyl-
methylamino)-3-[4-benzyloxy-3-(1-ethyl-1-methylallyl-
oxy)phenyl]-3-(tert-butyldimethylsilyloxy)-propionic acid
methyl ester (30). Phenol $\mathbf{4}$ (1 equiv) was dissolved in
dichloromethane $(1.5 \mathrm{~mL}$, degassed by bubbling argon for 20
min) at room temperature. $(1 S, 2 S)-1,2-B i s(2-$
diphenylphosphinobenzoyl)aminocyclohexane $\mathbf{1 9}(0.5$ equiv)
and tris (dibenzylideneacetone)dipalladium(0)-chloroform adduct ( 0.1 equiv) were added. The carbonate precursor to the $\pi$-allyl Pd complex (5 equiv) was added as a $200 \mu \mathrm{L}$ solution in dichloromethane. After $2 \mathrm{~h}$, the reaction mixture was loaded directly onto a silica gel column. Excess $\pi$-allyl precursor and dibenzylideneacetone were eluted using 20/1 hexanes/ethyl acetate. Desired product 30 eluted with 10/1 hexanes/ethyl acetate, followed by recovered 4 which eluted with $4 / 1$ hexanes/ethyl acetate. $\mathrm{R}_{\mathrm{f}}=0.5$ (5/1 hexanes/ethyl acetate); ${ }^{1} \mathbf{H}$ NMR $(500 \mathrm{MHz}$, DMSO$\mathrm{d}_{6}$ ) d 7.75-7.19 (mult, 13H), 6.31-6.22 (mult, $\left.1 \mathrm{H}\right), 5.60(5.53)(\mathrm{d}, \mathrm{J}=5 \mathrm{~Hz}, 1 \mathrm{H}), 5.42-5.05$ (mult, 5H), $3.91(3.87)(\mathrm{s}, 3 \mathrm{H}), 3.34(3.18(\mathrm{~s}, 3 \mathrm{H}), 2.00-1.90$ (mult, $2 \mathrm{H}), 1.47$ (1.51) (s, $3 \mathrm{H}), 1.14$ (t, J=9 Hz, 3H), 1.09 (1.08) (s, 9H), 0.28 (0.27) (s, 3H), $0.01(-0.01)(\mathrm{s}, 3 \mathrm{H})$. The NMR chemical shifts in parentheses are the shifts for the minor rotamer.

Using 16: 30 was prepared according to the above General Procedure from $16(8.7 \mathrm{mg}$, $0.0550 \mathrm{mmol}, 5$ equiv) to afford $\mathbf{3 0}(3.4 \mathrm{mg}, 0.00514 \mathrm{mmol}, 78 \%)$.

Using 17: 30 was prepared according to the above General Procedure from $17(8.7 \mathrm{mg}$, $0.0550 \mathrm{mmol}, 5$ equiv) to afford $\mathbf{3 0}(0.4 \mathrm{mg}, 0.000659 \mathrm{mmol}, 10 \%)$.

Using 31: 30 was prepared according to the above General Procedure from $\mathbf{3 1}(8.7 \mathrm{mg}$, $0.0335 \mathrm{mmol}, 5$ equiv) to afford $\mathbf{3 0}$ ( $1.0 \mathrm{mg}, 0.00151 \mathrm{mmol}, 24 \%)$.

Using 34: 30 was prepared according to the above General Procedure from 34 (3.6 mg, $0.00510 \mathrm{mmol}, 1$ equiv) to afford $\mathbf{3 0}(0.5 \mathrm{mg}, 0.000755 \mathrm{mmol}, 15 \%)$. 


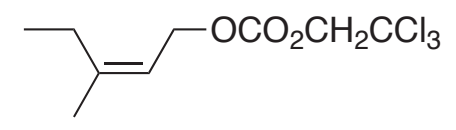

Carbonic acid (Z)-3-methyl-pent-2-enyl ester 2,2,2trichloro-ethyl ester (31). 3-Methylpent-2-en-1-ol (750 $\mathrm{mg}, 7.49 \mathrm{mmol}, 1$ equiv) was diluted with dichloromethane $(15 \mathrm{~mL})$ at $0{ }^{\circ} \mathrm{C}$. 2,2,2-Trichloroethyl chloroformate $(1.56 \mathrm{~mL}, 11.2 \mathrm{mmol}, 1.5$ equiv) was added followed by pyridine $(727 \mu \mathrm{L}, 8.99 \mathrm{mmol}, 1.2$ equiv). The reaction was complete immediately and saturated $\mathrm{NH}_{4} \mathrm{Cl}$ solution $(10 \mathrm{~mL})$ was added and the layers were separated. The aqueous layer was then extracted with dichloromethane $(3 \times 25$ $\mathrm{mL}$ ). The combined organic layers were dried over anhydrous $\mathrm{Na}_{2} \mathrm{SO}_{4}$. After careful removal of the solvent under reduced pressure, the crude mixture was loaded onto a silica gel column. Using a gradient of 20/1 pentane/diethyl ether to $100 \%$ diethyl ether, 31 was obtained (2.04 g, $7.42 \mathrm{mmol}, 99 \%) . \quad \mathrm{R}_{\mathrm{f}}=0.8$ (5/1 hexanes/ethyl acetate); IR (film) 2970 m, 1759 s, 1668 w, 1447 m, 1389 m, 1279 s, 1238 s, 1124 w, 1062 w, 945 w, 901 w, 818 m, $727 \mathrm{~m} ;{ }^{1} \mathbf{H}$ NMR $\left(500 \mathrm{MHz}, \mathrm{CDCl}_{3}\right) \delta 5.33(\mathrm{t}, \mathrm{J}=10 \mathrm{~Hz}, 1 \mathrm{H}), 4.72(\mathrm{~s}, 3 \mathrm{H}), 4.67$ (d, J=7 $\mathrm{Hz}, 2 \mathrm{H}), 2.10$ (q, J=8 Hz, 2H), $1.73(\mathrm{~s}, 3 \mathrm{H}), 0.98(\mathrm{t}, \mathrm{J}=7.5 \mathrm{~Hz}, 3 \mathrm{H}) ;{ }^{13} \mathbf{C}$ NMR $(125.5$ $\left.\mathrm{MHz}, \mathrm{CDCl}_{3}\right) \delta$ 153.9, 146.3, 116.9, 94.4, 76.6, 65.2, 25.0, 22.9, 12.9;

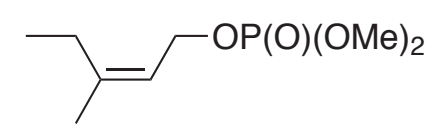

Phosphoric acid dimethyl ester (Z)-3-methyl-pent-2-enyl ester (32). 3-Methylpent-2-en-1-ol (347 mg, $3.47 \mathrm{mmol}, 1$ equiv) was diluted with dichloromethane $(10 \mathrm{~mL})$ at $0{ }^{\circ} \mathrm{C}$. Pyridine ( $672 \mu \mathrm{L}, 8.32 \mathrm{mmol}, 2.4$ equiv) was added followed by phosphorochloridic acid dimethyl ester (1.2 mL, $10.4 \mathrm{mmol}, 3.0$ equiv). The reaction was allowed to warm to room temperature and stirred $1.5 \mathrm{~h}$. $1 \mathrm{~N} \mathrm{HCl}$ solution $(10 \mathrm{~mL})$ was added and the layers were separated. The aqueous layer was then extracted with dichloromethane $(3 \times 20$ $\mathrm{mL}$ ). The combined organic layers were dried over anhydrous $\mathrm{Na}_{2} \mathrm{SO}_{4}$. After careful removal of the solvent under reduced pressure, the crude mixture was loaded onto a silica gel column. Using a gradient of 1/1 pentane/diethyl ether to $100 \%$ diethyl ether, 32 was obtained ( $279 \mathrm{mg}, 1.34 \mathrm{mmol}, 38 \%) . \mathrm{R}_{\mathrm{r}}=0.15$ (5/1 hexanes/ethyl acetate); IR (film) 2965 s, 1668 w, 1450 m, 1380 w, 1283 s, 1187 m, 1042 s, 987 s, 847 s, 751 w; ${ }^{1}$ H NMR (500 $\left.\mathrm{MHz}, \mathrm{CDCl}_{3}\right) \delta 5.15$ (t, J=7Hz, 1H), 3.55 (s, 3H), 3.55 (d, J=9 Hz, 2H), 3.52 (s, 3H), 1.90 (q, J=7.5 Hz, 2H), 1.55 (s, 3H), $0.79(\mathrm{t}, \mathrm{J}=8 \mathrm{~Hz}, 3 \mathrm{H}) ;{ }^{13} \mathbf{C}$ NMR $\left(125.5 \mathrm{MHz}, \mathrm{CDCl}_{3}\right) \delta$ 144.8, 118.1, 63.4, 53.7, 24.5, 22.4, 18.0, 12.4; HRMS calculated for $\mathrm{C}_{8} \mathrm{H}_{17} \mathrm{O}_{4} \mathrm{P}(\mathrm{M}+\mathrm{Na})$ : 231.0762, found (ESI): 231.0768 .

Chloro-3-methylpent-2-enyl formate (73). This compound was synthesized following the procedure for the synthesis of chloro-2trimethylsilylethyl formate ${ }^{7}$. 3-Methylpent-2-ene-1-ol $(50.5 \mathrm{mg}$, $0.504 \mathrm{mmol}, 1$ equiv) was dissolved in toluene $(0.3 \mathrm{~mL})$ at ambient temperature. Potassium carbonate ( $70.9 \mathrm{mg}, 0.513 \mathrm{mmol}, 1$ equiv) was added followed by phosgene ( $20 \%$ solution in toluene) $(370 \mu \mathrm{L}, 0.699 \mathrm{mmol}, 1.4$ equiv). The reaction was stirred for $1.5 \mathrm{~h}$ and then argon was bubbled for 5 min to blow away excess phosgene. The reaction mixture was filtered through a pad of anhydrous magnesium sulfate, and rinsed with ether. Ether was carefully removed under reduced pressure, and

\footnotetext{
${ }^{7}$ Shute, R. E.; Rich, D. H. Synthesis 1887, 346.
} 
the remaining toluene solution of chloro-3-methylpent-2-enyl formate $\mathbf{7 3}$ was used directly without characterization for the next reaction. $\mathrm{R}_{\mathrm{f}}=0.9(5 / 1$ hexanes/ethyl acetate).

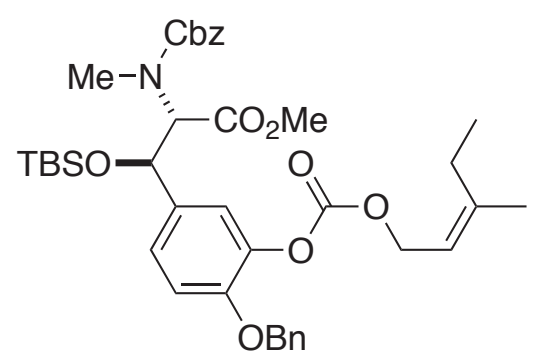

2-(Benzyloxycatbonylmethylamino)-3-[4benzyloxy-3-(3-methylpent-2-enyloxycarbonyloxy)phenyl]-3-(tert-butyldimethylsilyloxy)propionic acid methyl ester (34). Phenol $4(5.4 \mathrm{mg}, 0.0093$ mmol, 1 equiv) was dissolved in dichloromethane $(0.2$ $\mathrm{mL})$ at $0{ }^{\circ} \mathrm{C}$. Diisopropylethylamine $(8 \mu \mathrm{L}, 0.046$ mmol, 5 equiv) was added followed by $73(0.7 \mathrm{M}$ solution in toluene) (40 $\mu \mathrm{L}, 0.028 \mathrm{mmol}, 3$ equiv).

The reaction was allowed to warm to room temperature and stirred for $20 \mathrm{~h}$. The reaction was diluted with dichloromethane and saturated sodium bicarbonate solution was added. The product was extracted three times with dichloromethane and the combined organic layers were dried over anhydrous $\mathrm{MgSO}_{4}$. After removal of the solvent under reduced pressure, the crude product was purified using silica gel column chromatography. Using a gradient of $5 / 1$ to $2 / 1$ hexanes/ethyl acetate, 34 (3.9 $\mathrm{mg}, 0.0055 \mathrm{mmol}, 59 \%$ ) was obtained. $\mathrm{R}_{\mathrm{f}}=0.6$ (2/1 hexanes/ethyl acetate); ${ }^{1} \mathbf{H} \mathbf{~ N M R}\left(500 \mathrm{MHz}, \mathrm{CDCl}_{3}\right) \delta$ 7.40-6.75 (mult, 13H), 5.33-4.65 (mult, 7H), 3.67 (3.62) (s, 3H), 3.10 (3.11 (s, 3H), 2.11-2.05 (mult, 2H), 1.72 (s, 3H), 0.97 (0.96) (t, J=8 Hz, 3H), 0.84 (0.82) (s, 9H), 0.00 (-0.02) (s, $3 \mathrm{H}),-0.24(-0.26)(\mathrm{s}, 3 \mathrm{H})$; MS calculated for $\mathrm{C}_{39} \mathrm{H}_{51} \mathrm{O}_{9} \mathrm{NSi}(\mathrm{M}+\mathrm{Na})$ : 728 , found (ESI): 728. The NMR chemical shifts in parentheses are the shifts for the minor rotamer.

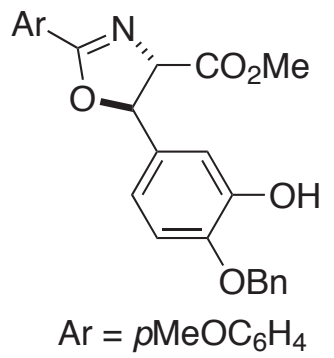

(4S,5R)-5-(4-Benzyloxy-3-hydroxyphenyl)-2-(4-methoxyphenyl)-4,5-dihydrooxazole-4-(carboxylic acid methyl ester) (40). Fully protected 10 (950 $\mathrm{mg}, 2.00 \mathrm{mmol}, 1$ equiv) was dissolved in THF $(30 \mathrm{~mL})$ and methanol $(6 \mathrm{~mL})$ at $0{ }^{\circ} \mathrm{C}$. Potassium carbonate (332 $\mathrm{mg}, 2.40 \mathrm{mmol}, 1.2$ equiv) was added and the reaction was allowed to warm to room temperature and stirred overnight. Most of the solvent was removed under reduced pressure, and the reaction was resuspended in dichloromethane. $\mathrm{pH}$ 2.5 Phosphate buffer was added until the yellow color disappeared (about $12 \mathrm{~mL}$ ) and the layers were separated. The aqueous layer was extracted twice more with dichloromethane and the combined organic layers were dried over anhydrous $\mathrm{Na}_{2} \mathrm{SO}_{4}$. After the removal of the solvent under reduced pressure, the products were purified using silica gel column chromatography. Using a gradient of $2 / 1$ to $1 / 2$ hexanes/ethyl acetate, 40 (769 mg, $1.78 \mathrm{mmol}, 89 \%)$ was obtained followed by the cis isomer, $(4 R, 5 R)-5-(4-$ benzyloxy-3-hydroxyphenyl)-2-(4-methoxyphenyl)-4,5-dihydrooxazole-4-(carboxylic acid methyl ester) (41.5 mg, $0.0957 \mathrm{mmol}, 5 \%) . \quad \mathrm{R}_{\mathrm{f}}=0.4$ (2/1 benzene/ether); IR (film) 3028 w, 2952 w, 1739 m, 1637 m, 1609 m, 1512 s, 1452 w, 1421 w, 1339 m, 1257 s, 1228 s, 1171 s, 1130 w, 1084 m, 1025 m, 913 w, 842 w, 743 w, 696 w; ${ }^{1}$ H NMR (500 $\left.\mathrm{MHz} \mathrm{CDCl}_{3}\right) \delta 7.96$ (dd, J=2, $7 \mathrm{~Hz}, 2 \mathrm{H}$ ), 7.38-7.31 (mult, 5H), 6.94 (d, J=2 Hz, 1H), $6.88(\mathrm{~d}, \mathrm{~J}=7 \mathrm{~Hz}, 2 \mathrm{H}), 6.87$ (d, J=8 Hz, 1H), 6.81 (dd, J=2, $8 \mathrm{~Hz}, 1 \mathrm{H}), 6.32$ (s, 1H), 5.73 $(\mathrm{d}, \mathrm{J}=7.5 \mathrm{~Hz}, 1 \mathrm{H}), 5.07(\mathrm{~s}, 2 \mathrm{H}), 4.73(\mathrm{~d}, \mathrm{~J}=7.5 \mathrm{~Hz}, 1 \mathrm{H}), 3.81(\mathrm{~s}, 3 \mathrm{H}), 3.78(\mathrm{~s}, 3 \mathrm{H}) ;{ }^{13} \mathrm{C}$ NMR $\left(125.5 \mathrm{MHz}, \mathrm{CDCl}_{3}\right) \delta 171.4,165.2,162.5,146.4,146.1,136.0,133.0,130.4$, 
128.6, 128.3, 127.6, 119.1, 117.5, 113.6, 112.3, 112.2, 82.8, 76.4, 71.0, 55.3, 52.7; HRMS calculated for $\mathrm{C}_{25} \mathrm{H}_{25} \mathrm{NO}_{6}(\mathrm{M}+\mathrm{H}): 434.1598$, found (MALDI): 434.1592 .

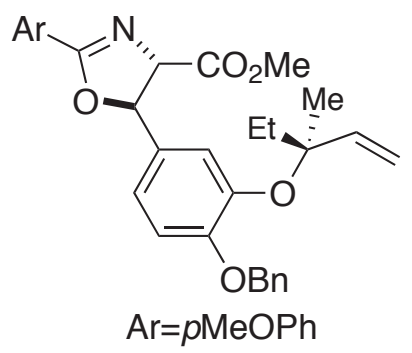
(4S,5R)-5-[3-(Benzyloxy-4-)-1-(1S)-ethyl-1-methylallyloxy)- phenyl]-2-(4-methoxyphenyl)-4,5-dihydrooxazole-4- carboxylic acid methyl ester (41b).

Phenol 40 (827 mg, $1.91 \mathrm{mmol}, 1$ equiv) was dissolved in dichloromethane (degassed by bubbling argon for $20 \mathrm{~min})(8$ $\mathrm{mL})$ at room temperature. $(1 R, 2 R)$-1,2-bis(2-diphenylphosphinobenzoyl)aminocyclohexane $19(331 \mathrm{mg}, 0.480$ mmol, 0.25 equiv) and tris(dibenzylideneacetone)dipalladium(0)-chloroform adduct $(99.5 \mathrm{mg}, 0.0961 \mathrm{mmol}, 0.05$ equiv) were added. Carbonic acid-1-ethyl-1-methylallyl ester methyl ester 16 (907 mg, 5.73 mmol, 3 equiv) was added dropwise over $10 \mathrm{~min}$. The reaction turned yellow. When $\mathbf{1 6}$ was fully consumed, the reaction color changed to orange. A subsequent aliquot of $16(50 \mu \mathrm{L}, 0.3$ mmol, 0.15 equiv) was added when the color change was observed. (About one addition every $5 \mathrm{~min}$ over $20 \mathrm{~min}$ were necessary.) Though the reaction appeared to stop after one hour, the contents were further stirred for two additional hours and then were directly loaded onto a silica gel column. A gradient of 10/1 to 5/1 hexanes/ethyl acetate eluted excess 16 and dibenzylideneacetone. The product $\mathbf{4 1 b},(837 \mathrm{mg}, 1.55 \mathrm{mmol}, 81 \%$, $27 \%$ de) eluted with $2 / 1$ hexanes/ethyl acetate and recovered starting material $40(130 \mathrm{mg}$, $0.282 \mathrm{mmol}, 15 \%)$ was obtained upon elution with $1 / 2$ hexanes/ethyl acetate. $\mathrm{R}_{\mathrm{f}}=0.4(2 / 1$ hexanes/ethyl acetate); $\mathrm{Rt}=31.6 \mathrm{~min}$ (major isomer 41b), $37.4 \mathrm{~min}$ (minor isomer 41a), Chiralpak AD (0.9 mL/min, 80\% ethanol/hexane); IR (film) 2969 w, 2935 w, $1740 \mathrm{~m}$, $1641 \mathrm{~m}, 1609 \mathrm{~m}, 1576 \mathrm{w}, 1512 \mathrm{~s}, 1454 \mathrm{w}, 1434 \mathrm{w}, 1420 \mathrm{w}, 1307 \mathrm{w}, 1258 \mathrm{~s}, 1224 \mathrm{~m}$, 1170 s, 1133 w, 1082 m, 1026 w, 1008 w, 920 w, 842 w, 772 w, 740 w, 697 w; ${ }^{1}$ H NMR $\left(500 \mathrm{MHz}, \mathrm{CDCl}_{3}\right) \delta 7.96$ (dd, J=2, $\left.9 \mathrm{~Hz}, 2 \mathrm{H}\right), 7.42$ (d, J=9 Hz, 1H), 7.35 (t, J=7.5 Hz, $2 \mathrm{H}$ ), 7.29 (t, J=7.5 Hz, 1H), 6.93-6.90 (mult, 2H), 6.91 (d, J=9 Hz, 2H), 6.01 (dd, J=12, $18 \mathrm{~Hz}, 1 \mathrm{H}), 5.71$ (d, J=7 Hz, 1H), 5.06 (s, 2H), 5.05-5.00 (mult, 2H), 4.70 (d, J=7 Hz, $1 \mathrm{H}), 3.75(\mathrm{~s}, 3 \mathrm{H}), 3.72$ (s, 3H), 1.83-1.71 (mult, 2H), $1.23(\mathrm{~s}, 3 \mathrm{H}), 0.91$ (t, J=7.5 Hz, 3H); ${ }^{13} \mathbf{C}$ NMR $\left(100 \mathrm{MHz}, \mathrm{CDCl}_{3}\right) \delta 171.4,165.1,162.4,152.0,146.2,142.9,137.1,132.0$, 130.4, 128.3, 127.6, 127.1, 120.2, 120.1, 119.2, 114.8, 114,5, 113.6, 83.6, 82.7, 76.6, 71.0, 55.2, 52.6, 34.1, 21.1, 8.2; HRMS calculated for $\mathrm{C}_{31} \mathrm{H}_{33} \mathrm{NO}_{6}(\mathrm{M}+\mathrm{H}): 516.2381$, found (MALDI): 516.2385 .

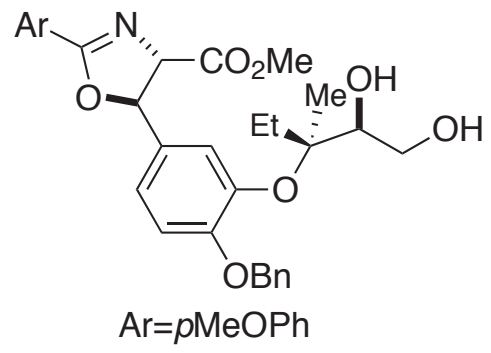

(4S,5R)-5-[4-Benzyloxy-3-((1S,2S)-1-ethyl-2,3dihydroxy-1-methylpropoxy)phenyl-4,5dihydrooxazole-4-carboxylic acid methyl ester (42d). Terminal alkene $41 \mathrm{~b}$ (779 $\mathrm{mg}, 1.51 \mathrm{mmol}, 1$ equiv), potassium carbonate $(210 \mathrm{mg}, 1.52 \mathrm{mmol}, 1$ equiv), sodium bicarbonate ( $256 \mathrm{mg}, 3.05 \mathrm{mmol}, 2$ equiv), potassium ferricyanide(III) (1.49 g, $4.54 \mathrm{mmol}, 3$ equiv) were charged in a flask. (DHQD) ${ }_{2} \mathrm{PYR}(266 \mathrm{mg}, 0.302$ mmol, 0.2 equiv) was added as a solution in tert-butyl alcohol $(7.5 \mathrm{~mL})$, followed by potassium osmate dihydrate $(28.1 \mathrm{mg}, 0.0763 \mathrm{mmol}, 0.05$ equiv) in water $(7.5 \mathrm{~mL})$. The reaction was stirred at room temperature for 3 days. Sodium sulfite $(2.27 \mathrm{~g})$ was added 
and the reaction was stirred for $30 \mathrm{~min}$. The products were extracted three times with ethyl acetate. The combined organic layers were washed with brine and dried over anhydrous $\mathrm{Na}_{2} \mathrm{SO}_{4}$. After removal of the solvent under reduced pressure, the crude material was purified using silica gel column chromatography. Recovered starting material 41b (118 mg, $0.227 \mathrm{mmol}, 15 \%)$ eluted using 3/1 hexanes/ethyl acetate. The desired product 42d (614 mg, $1.12 \mathrm{mmol}, 74 \%, 78 \% \mathrm{de})$ was obtained by elution with a gradient of $1 / 1$ to $1 / 4$ hexanes/ethyl acetate. $R_{\mathrm{f}}=0.3$ ( $1 / 2$ hexanes/ethyl acetate); IR (film) 3483 m, 2940 m, 1742 m, 1634 s, 1608 s, 1579 w, 1511 s, 1505 s, 1455 w, 1436 w, 1422 w, 1377 w, 1259 s, 1224 m, 1171 s, 1133 m, 1083 m, 1026 m, 842 w, 749 w, 698w; ${ }^{1} \mathbf{H}$ NMR $\left(400 \mathrm{MHz}, \mathrm{CDCl}_{3}\right) \delta 8.01(\mathrm{~d}, \mathrm{~J}=12 \mathrm{~Hz}, 2 \mathrm{H}), 7.45-7.23$ (mult, 5H), $6.96(\mathrm{~d}, \mathrm{~J}=12$ $\mathrm{Hz}, 2 \mathrm{H}$ ), 7.08-6.90 (mult, 3H), 5.76 (d, J=8 Hz, 1H), 5.09 (s, 2H), 4.74 (d, J=8 Hz, 1H), 3.87 (s, 3H), 3.85 (s, 3H), 3.60-3.76 (mult, 3H), 1.97-1.79 (mult, 1H), 1.68-1.45 (mult, $1 \mathrm{H}), 1.19(\mathrm{~s}, 3 \mathrm{H}), 0.98(\mathrm{t}, \mathrm{J}=8 \mathrm{~Hz}, 3 \mathrm{H}) ;{ }^{13} \mathbf{C} \mathbf{N M R}\left(100 \mathrm{MHz}, \mathrm{CDCl}_{3}\right) \delta 171.4,165.2$, $162.6,152.7,143.9$ (144.0), 135.7, 132.5 (135.6), 130.4, 128.7, 128.6, 128.4, 127.7 (127.9), 122.3 (122.9), 121.6 (121.8), 119.1, 113.8, 87.3 (86.8), 82.5, 76.6 (75.4), 74.1, 71.2 (71.3), 62.6 (62.5), 55.4, 29.5 (29.3), 19.7 (19.9), 8.4; HRMS calculated for $\mathrm{C}_{31} \mathrm{H}_{35} \mathrm{NO}_{8}(\mathrm{M}+\mathrm{Na}): 572.2255$, found (MALDI): 572.2269. The chemical shifts in parentheses are the shifts for the minor diastereomer.

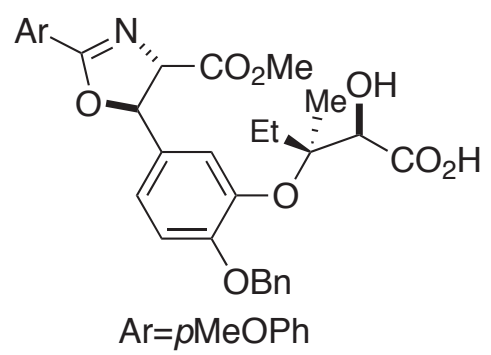

$(4 S, 5 R)-5-\{4-B e n z y l o x y-3-[(1 S, 2 R)-1-$ (carboxyhydroxymethyl)-1-methylpropoxy]phenyl $\}-2-$ (4-methoxyphenyl)-4,5-dihydrooxazole-4-carboxylic acid methyl ester (43). Diol 42d (481 mg, $0.876 \mathrm{mmol}$, 1 equiv) was dissolved in dichloromethane $(22 \mathrm{~mL})$ at 0 ${ }^{\circ} \mathrm{C}$. Saturated sodium bicarbonate solution $(22 \mathrm{~mL})$ was added followed by the addition of potassium bromide (20.4 mg, $0.171 \mathrm{mmol}, 0.2$ equiv), 2,2,6,6-tetramethyl-1piperidinyloxy, free radical (TEMPO) $(28.1 \mathrm{mg}, 0.180 \mathrm{mmol}, 0.2$ equiv). Sodium hypochlorite solution (commercial bleach, $5.25 \%$ by weight, $1.5 \mathrm{~mL}, 1.1 \mathrm{mmol}, 1.2$ equiv) was added with vigorous stirring. The reaction was complete within 20 min. Saturated sodium sulfite solution was added to quench excess oxidant. The layers were separated and the aqueous layer was extracted twice more with dichloromethane. The combined organic layers were dried over anhydrous $\mathrm{Na}_{2} \mathrm{SO}_{4}$. The solvent was removed under reduced pressure and the crude product was directly used for the next oxidation reaction without further characterization. $\mathrm{R}_{\mathrm{f}}=0.8(1 / 2$ hexanes/ethyl acetate).

The crude aldehyde was dissolved in tert-butyl alcohol $(47 \mathrm{~mL})$ and 2-methyl-2butene $(18 \mathrm{~mL})$ at room temperature. Sodium chlorite $(913 \mathrm{mg}, 8.08 \mathrm{mmol}, 9.2$ equiv, $\sim 80 \%$ tech grade) and sodium phosphate monobasic hydrate $(836 \mathrm{mg}, 6.06 \mathrm{mmol}, 6.9$ equiv) were dissolved in water $(16 \mathrm{~mL})$ and added as an aqueous solution. After stirring for $20 \mathrm{~min}, \mathrm{pH} 2.5$ phosphate buffer was added and the layers were separated. The aqueous layer was extracted twice with chloroform. The combined organic layers were dried over anhydrous $\mathrm{Na}_{2} \mathrm{SO}_{4}$. After removal of the solvent, the crude product was purified using silica gel column chromatography. TEMPO eluted first with $1 / 1$ hexanes/ethyl acetate. 43 (494 mg, $0.876 \mathrm{mmol}$, quant.) started to elute using 1/2 hexanes/ethyl acetate and continued to elute until elution with 10/1 chloroform/methanol. $\mathrm{R}_{\mathrm{f}}=0.5$ (5/1 chloroform/methanol); IR (film) $3478 \mathrm{w}, 2953 \mathrm{~m}, 2566 \mathrm{w}, 1742 \mathrm{~s}, 1634 \mathrm{~s}$, 
1609 s, 1511 s, 1505 s, 1455 m, 1309 m, 1259 s, 1224 s, 1173 s, 1131 m, 1086 m, 1026 m, 949 w, 919 w, 842 w, 753 m, 698 w; ${ }^{1} \mathbf{H}$ NMR (400 MHz, CDCl ${ }_{3}$ ) 8.99 (d, J=12 Hz, 2H), 7.47-7.31 (mult, 5H), 6.95 (d, J=12 Hz, 2H), 7.03-6.91 (mult, 3H), 5.78 (d, J=8 Hz, $1 \mathrm{H}), 5.12$ (5.14) (s, 2H), $4.75(\mathrm{~d}, \mathrm{~J}=8 \mathrm{~Hz}, 1 \mathrm{H}), 4.33$ (4.26) (br, 1H), 3.87 (s, 3H), 3.84 (s, $3 \mathrm{H}), 2.02-1.72$ (mult, 2H), 1.23 (1.25) (s, 3H), 1.03 (t, J=8 Hz, 3H); ${ }^{13} \mathbf{C}$ NMR (100 MHz, $\left.\mathrm{CDCl}_{3}\right) \delta 171.3,165.3,162.7,152.2,143.2,135.2,132.8,130.5,128.8,128.7,128.5$, 128.1, 127.9 (127.7), 122.3, 122.2 (121.6), 118.9, 113.8 (113.6), 86.7 (86.4), 82.4, 76.5, $74.5,71.2$ (71.1), 55.4, 52.8, $28.9,19.8$ (19.2), 8.0 (7.6); HRMS calculated for $\mathrm{C}_{31} \mathrm{H}_{35} \mathrm{NO}_{9}(\mathrm{M}+\mathrm{H}): 564.2228$, found (MALDI): 564.2220. The chemical shifts in parentheses are the shifts for the minor diastereomer.

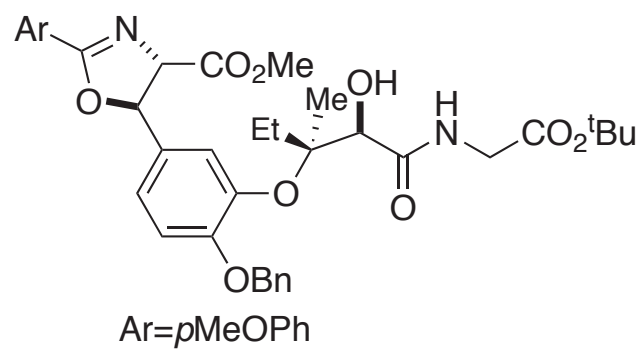

$(4 S, 5 R)-5-(4-B e n z y l o x y-3-\{(1 S)-1-[(R)-(t e r t-$ butoxycarbonylmethylcarbamoyl)hydroxyme thyl]-1-methylpropoxy\}phenyl)-2-(4methoxyphenyl)-4,5-dihydrooxazole-4carboxylic acid methyl ester (44). Free acid 43 (285 mg, $0.506 \mathrm{mmol}, 1$ equiv) and glycine tert-butyl ester hydrochloride (132 $\mathrm{mg}, 0.788$ mmol, 1.5 equiv) were dissolved in acetonitrile

$(20 \mathrm{~mL})$ at room temperature. Benzotriazole-1-yloxy-tris(dimethylamino)phosphonium hexafluorophosphate (BOP reagent) $(450 \mathrm{mg}, 1.02 \mathrm{mmol}, 2$ equiv) was added, followed by the addition of diisopropylethylamine ( $440 \mu \mathrm{L}, 2.53 \mathrm{mmol}, 5$ equiv). After stirring for 1 hour, more glycine tert-butyl ester hydrochloride (39.3 mg, $0.234 \mathrm{mmol}, 0.5$ equiv), BOP reagent (112 mg, $0.253 \mathrm{mmol}, 0.5$ equiv) and diisopropylethylamine ( $88 \mu \mathrm{L}, 0.51$ mmol, 1 equiv) were added. The reaction was further stirred for another $2.5 \mathrm{~h}$ and then diluted with ethyl acetate. Phosphate buffer $(\mathrm{pH} 2.5)$ was added and the layers were separated. The aqueous layer was extracted twice more with ethyl acetate and the combined organic layers were washed twice with saturated sodium bicarbonate solution, once with brine, and dried over anhydrous sodium sulfate. After the removal of the solvent under reduced pressure, the crude product was purified using silica gel column chromatography. Using a gradient of $2 / 1$ to $1 / 2$ hexanes/ethyl acetate, 44 (300 $\mathrm{mg}, 0.443$ mmol, 88\%) was obtained. $\mathrm{R}_{\mathrm{f}}=0.5$ (1/1 hexanes/ethyl acetate); IR (film) $3363 \mathrm{w}, 2976$ w, 2930 w, 1741 s, 1672 m, $1641 \mathrm{~m}, 1609$ m, 1512 s, 1455 w, 1434 w, 1367 w, 1257 s, 1224 m, 1169 s, 1134 m, 1082 m, 1026 w, 1009 w, 843 w, 750 w, 698 w; ${ }^{1}$ H NMR (500 $\mathrm{MHz}, \mathrm{CDCl}_{3}$ ) $\delta 7.97$ (dd, J=2, $\left.7 \mathrm{~Hz}, 2 \mathrm{H}\right), 7.74$ (t, J=6 Hz, 1H), 7.37-7.32 (mult, 5H), 7.07 $(\mathrm{d}, \mathrm{J}=2 \mathrm{~Hz}, 1 \mathrm{H}), 6.99$ (dd, J=2, $8.5 \mathrm{~Hz}, 1 \mathrm{H}), 6.94(\mathrm{~d}, \mathrm{~J}=7,5 \mathrm{~Hz}, 2 \mathrm{H}), 6.91(\mathrm{~d}, \mathrm{~J}=8.5 \mathrm{~Hz}$, $1 \mathrm{H}), 5.73(\mathrm{~d}, \mathrm{~J}=7.6 \mathrm{~Hz}, 1 \mathrm{H}), 5.03(\mathrm{dd}, \mathrm{J}=10,22 \mathrm{~Hz}, 2 \mathrm{H}), 4.71(4.72)(\mathrm{d}, \mathrm{J}=7.5 \mathrm{~Hz}, 1 \mathrm{H})$, 4.26 (4.18) (d, J=3.5 Hz, 1H), 3.83 (s, 3H), 3.82 (s, 3H), 3.57 (dd, J=6, $18 \mathrm{~Hz}, 1 \mathrm{H}), 3.36$ (dd, J=5.5, $18 \mathrm{~Hz}, 1 \mathrm{H}), 1.95$ (mult, 1H), 1.80 (mult, 1H), 1.36 (s, 9H), $1.24(1.42)(\mathrm{s}, 3 \mathrm{H})$, $0.98(\mathrm{t}, \mathrm{J}=7 \mathrm{~Hz}, 3 \mathrm{H}) ;{ }^{13} \mathbf{C} \mathbf{N M R}\left(100 \mathrm{MHz}, \mathrm{CDCl}_{3}\right) \delta 172.2$ (171.1), 168.4, 165.2, 162.6, 152.6 (151.4), 143.9, 135.8 (135.6), 132.7 (132.6), 130.4, 128.9, 128.7 (128.5), 127.7 (127.9), 122.4, 120.5 (121.7), 119.1 (119.8), 113.8, 113.6 (113.4), 86.1 (87.0), 82.5 (82.4), 81.6 (81.9), 76.8, 72.9 (74.8), 71.2 (71.1), 55.4, 52.8, 41.6 (41.7), 28.5 (28.9), 27.9, (28.0), 18.9 (19.3), 8.0 (7.6); HRMS calculated for $\mathrm{C}_{37} \mathrm{H}_{44} \mathrm{~N}_{2} \mathrm{O}_{10}(\mathrm{M}+\mathrm{Na}): 699.2888$, found (MALDI): 699.2905. The chemical shifts in parentheses are the shifts for the minor diastereomer. 


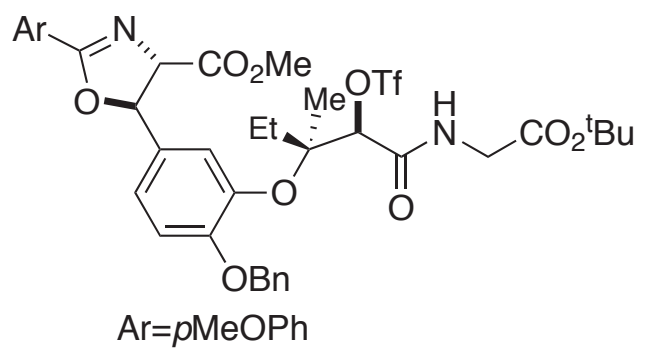

$(4 S, 5 R)-5-(4-B e n z y l o x y-3-\{(1 S)-1-[(R)-(t e r t-$ butoxycarbonylmethyl-carbamoyl)trifluoromethanesulfonyloxy-methyl]-1methyl-propoxy\}-phenyl)-2-(4-methoxyphenyl)-4,5-dihydro-oxazole-4-carboxylic acid methyl ester (45). Alcohol $44(1.23 \mathrm{mg}$, $1.81 \mathrm{mmol}, 1$ equiv) was dissolved in dichloromethane $(45 \mathrm{~mL})$ at $-20{ }^{\circ} \mathrm{C}$. Pyridine $(1.5 \mathrm{~mL}, 19 \mathrm{mmol}, 10$ equiv) was added followed by the addition of trifluoromethanesulfonic anhydride $(914 \mu \mathrm{l}, 5.43 \mathrm{mmol}, 3$ equiv). Temperature was carefully maintained at $-20^{\circ} \mathrm{C}$ over $8 \mathrm{~h}$. The reaction was then diluted with dichloromethane and saturated sodium bicarbonate solution was added and warmed to room temperature. The layers were separated and the aqueous layer was extracted twice more with dichloromethane. The combined organic layers were dried over anhydrous $\mathrm{Na}_{2} \mathrm{SO}_{4}$. After the removal of the solvent under reduced pressure, the crude triflate was purified using silica gel column chromatography. Byproduct and pyridine eluted using $3 / 1$ hexanes/ethyl acetate, followed by the desired triflate $(1.17 \mathrm{~g}$, $1.45 \mathrm{mmol}, 80 \%$ ), which eluted with a gradient of $3 / 1$ to $2 / 1$ hexanes/ethyl acetate. Recovered starting material 44 (164 mg, $0.242 \mathrm{mmol}$, 13\%) eluted using $1 / 2$ hexanes/ethyl acetate. 45 was used without further characterization. $\mathrm{R}_{\mathrm{f}}=0.6(1 / 1$ hexanes/ethyl acetate).

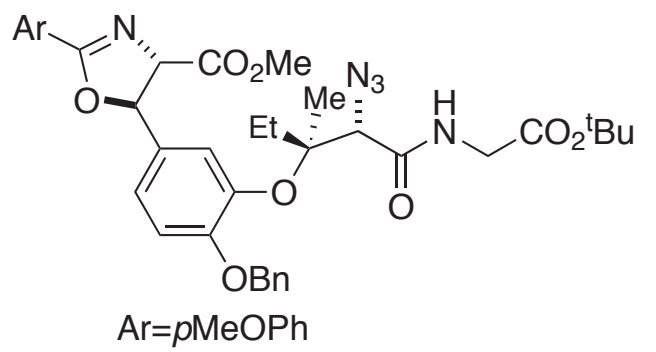

$(4 S, 5 R)-5-(3-\{(1 S)-1-[(S)-$ Azido- $($ tertbutoxycarbonymethylcarbamoyl)methyl]-1methylpropoxy\}-4-benzyloxyphenyl)-2-(4methoxyphenyl)-4,5-dihydrooxazole-4carboxylic acid methyl ester (46). Triflate 45 $(1.17 \mathrm{~g}, 1.45 \mathrm{mmol}, 1$ equiv) was dissolved in 1,3-dimethyl-3,4,5,6-tetrahydro-2(H)pyrimidinone (DMPU) $(6 \mathrm{~mL})$. Lithium azide (1.05 g, $12.0 \mathrm{mmol}, 8.3$ equiv, 56\% by weight dispersed in DMPU) was added and the reaction was stirred at room temperature for $2 \mathrm{~d}$. The reaction was loaded directly onto a silica gel column and eluted with $1 / 2$ hexanes/ethyl acetate to remove most of the reaction solvent, DMPU. The product fractions were concentrated and the crude product was purified using silica gel column chromatography. Trace amount of the recovered triflate eluted using a gradient of $3 / 4 / 2$ to $3 / 8 / 4$ hexanes/benzene/ether. The product $\mathbf{4 6}$ (711 $\mathrm{mg}, 1.01 \mathrm{mmol}, 70 \%$ ) eluted using a gradient of $3 / 8 / 4$ to $0 / 2 / 1$ hexanes/benzene/ether. $\mathrm{R}_{\mathrm{f}}=0.6$ ( $2 / 1$ benzene/ether, developed twice); IR (film) $3359 \mathrm{w}$, 2972 w, 2116 s, 1742 m, 1682 w, 1652 w, 1607 m, 1514 s, 1370 m, 1259 s, 1223 m, 1168 m, 1080w, 1026 w, 843 w, 770 w; ${ }^{1} \mathbf{H}$ NMR $\left(500 \mathrm{MHz}, \mathrm{CDCl}_{3}\right) \delta 7.98$ (d, J=9 Hz, 2H), 7.39-7.33 (mult, 6H), 7.05 (d, J=2 Hz, 1H), 7.03 (dd, J=2, $9 \mathrm{~Hz}, 1 \mathrm{H}$ ), 6.96-6.91 (mult, 1H), 6.92 (dd, J=2, $7 \mathrm{~Hz}, 2 \mathrm{H}), 5.75(\mathrm{~d}, \mathrm{~J}=7.5 \mathrm{~Hz}, 1 \mathrm{H}), 5.04(5.07)(\mathrm{d}, \mathrm{J}=2 \mathrm{~Hz}, 2 \mathrm{H}), 4.73$ (d, J=7.5 Hz, 1H), $4.33(4.28)(\mathrm{s}, 1 \mathrm{H}), 3.85(\mathrm{~s}, 3 \mathrm{H}), 3.83(\mathrm{~s}, 3 \mathrm{H}), 3.72(3.71)(\mathrm{dd}, \mathrm{J}=5.5$, $9.5 \mathrm{~Hz}, 1 \mathrm{H}), 3.61(3.54)(\mathrm{dd}, \mathrm{J}=5.5,18 \mathrm{~Hz}, 1 \mathrm{H}), 1.90-1.82$ (mult, 1H), 1.79-1.70 (mult, $1 \mathrm{H}), 1.39$ (1.41) (s, 9H), $1.30(1.20)(\mathrm{s}, 3 \mathrm{H}), 0.95(0.96)(\mathrm{t}, \mathrm{J}=7.5 \mathrm{~Hz}, 3 \mathrm{H}) ;{ }^{13} \mathbf{C}$ NMR (100 
$\left.\mathrm{MHz}, \mathrm{CDCl}_{3}\right) \delta 171.4,168.4,167.8,165.2,162.6,152.3$ (150.9), 143.5, 136.0, 132.3, 130.4, 128.8, 128.7, 128.4 (128.3), $127.8,122.9$ (123.0), 121.8 (121.9), 121.5, 119.1, 113.7, 113.6, 85.7 (85.9), 82.5, 81.9 (82.0), 70.9, 68.8 (69.2), 55.4, 52.8, 41.6 (41.8), 29.2, 27.9, 20.3 (20.1), 8.0 (8.3); HRMS calculated for $\mathrm{C}_{37} \mathrm{H}_{43} \mathrm{~N}_{5} \mathrm{O}_{9}(\mathrm{M}+\mathrm{H})$ : 702.3133, found (MALDI): 702.3163. The chemical shifts in parentheses are the shifts for the minor diastereomer.

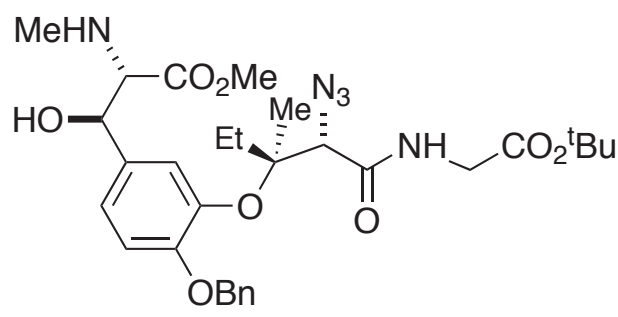

$(2 S, 3 R)-3-(3-\{(1 S)-1-[(S)-$ Azido- $($ tertbutoxycarbonylmethylcarbamoyl)methyl]-1methylpropoxy\}-4-benzyloxyphenyl)-3hydroxy-2-methylaminopropionic acid methyl ester (47). Oxazoline 46 (297 mg, $0.423 \mathrm{mmol}$, 1 equiv) was dissolved in dichloromethane $(4.5$ $\mathrm{mL}$ ) at room temperature. Methyl

trifluoromethanesulfonate $(57 \mu \mathrm{L}, 0.50 \mathrm{mmol}, 1.2$ equiv, freshly distilled from calcium hydride) was added and the reaction was stirred at room temperature for $1 \mathrm{~h}$. Toluene was added and the reaction mixture was azeotroped. The reaction mixture was redissolved in dichloromethane $(4.5 \mathrm{~mL})$ at $0{ }^{\circ} \mathrm{C}$. Saturated sodium bicarbonate solution $(4.5 \mathrm{~mL})$ and sodium borohydride $(32.4 \mathrm{mg}, 0.856 \mathrm{mmol}, 2$ equiv) were added at once with vigorous stirring, accompanied by bubbling. After $15 \mathrm{~min}$, the layers were separated and the aqueous layer was extracted twice more with dichloromethane. The combined organic layers were dried over anhydrous $\mathrm{Na}_{2} \mathrm{SO}_{4}$. After the removal of the solvent under reduced pressure, the crude products were purified using silica gel column chromatography. Using a gradient of $3 / 1$ to $3 / 2$ hexanes/ethyl acetate, the desired $(4 S, 5 R)-5-(3-\{(1 S)-1-[(S)$-azido-(tert-butoxycarbonylmethylcarbamoyl)methyl]-1methylpropoxy\}-4-benzyloxyphenyl)-2-(4-methoxyphenyl)-3-methyloxazolidine-4carboxylic acid methyl ester was obtained as a mixture with the over-reduced product, $(2 S, 3 R)-3-(3-\{(1 S)-1-[(S)$-azido-(tert-butoxycarbonylmethylcarbamoyl)methyl]-1methylpropoxy\}-4-benzyloxyphenyl)-3-hydroxy-2-[(4methoxybenzyl)methylamino]propionic acid methyl ester (251 $\mathrm{mg}$ combined). Using $1 / 2$ hexanes/ethyl acetate, starting material $46(29.7 \mathrm{mg}, 0.0416 \mathrm{mmol}, 10 \%)$ was recovered. $\mathrm{R}_{\mathrm{f}}=0.8(1 / 2$ hexanes/ethyl acetate). The NMR spectrum of this compound was too complex to be analyzed due to the presence of multiple oxazolidine diastereomers.

The mixture of $(4 S, 5 R)-5-(3-\{(1 S)-1-[(S)$-azido-(tert-butoxycarbonylmethylcarbamoyl)methyl]-1-methylpropoxy\}-4-benzyloxyphenyl)-2-(4-methoxyphenyl)-3-methyloxazolidine-4-carboxylic acid methyl ester and the over-reduced $(2 S, 3 R)-3-(3-\{(1 S)-1$ [(S)-azido-(tert-butoxycarbonylmethylcarbamoyl)methyl]-1-methylpropoxy\}-4-benzyloxyphenyl)-3-hydroxy-2-[(4-methoxybenzyl)methylamino]propionic acid methyl ester (251 mg combined) was dissolved in THF $(15 \mathrm{~mL}$ ) at room temperature. $0.1 \mathrm{~N}$ Oxalic acid solution $(12 \mathrm{~mL})$ was added and the reaction was allowed to stir overnight. After removal of THF at reduced pressure, aqueous $1 \mathrm{~N} \mathrm{HCl}(2 \mathrm{~mL})$ was added and the reaction mixture was washed with ether to remove $p$-methoxybenzaldehyde. The aqueous layer was then made basic to $\mathrm{pH}$ paper using saturated sodium bicarbonate solution. The products were extracted three times with ethyl acetate. The combined organic layers were dried over anhydrous $\mathrm{Na}_{2} \mathrm{SO}_{4}$ and the solvent was removed under reduced pressure and purified by elution with $2 / 1$ hexanes/ethyl acetate separated the 
over-reduced byproduct and some remaining $p$-methoxybenzaldehyde. Using a gradient of $1 / 2$ hexanes/ethyl acetate to $100 \%$ ethyl acetate to $10 / 1$ chloroform/ethyl acetate, the desired 47 (154 mg, $0.257 \mathrm{mmol}, 61 \%$ over 2 steps) was obtained. $\mathrm{R}_{\mathrm{f}}=0.4$ (ethyl acetate); ${ }^{1} \mathbf{H}$ NMR (500 MHz, $\mathrm{CDCl}_{3}$ ) $\delta$ 7.44-7.29 (mult, 6H), 7.07-6.86 (mult, 3H), 5.05 (5.02) (s, 2H), 4.53 (d, J=8 Hz, 1H), 4.29 (4.33) (s, 1H), 3.77-3.64 (3.21-3.18) (mult, 2H), 3.58 (3.59) (s, 3H), 3.53 (3.52) (s, 3H), 2.40 (s, 3H), 1.91-1.75 (mult, 2H), 1.39 (1.41) (s, 9H), $1.30(1.20)(\mathrm{s}, 3 \mathrm{H}), 1.00(0.97)(\mathrm{t}, \mathrm{J}=8 \mathrm{~Hz}, 3 \mathrm{H})$. The chemical shifts in parentheses are the shifts for the minor diastereomer.

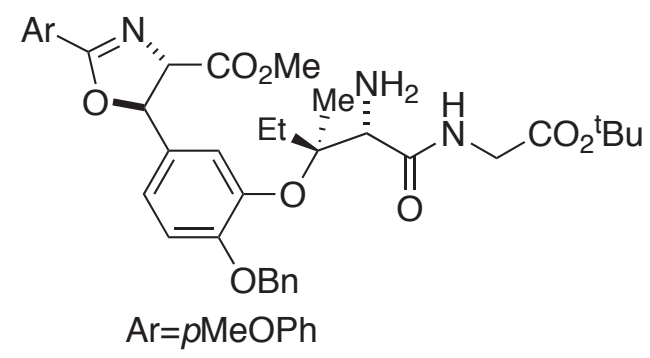

$(4 S, 5 R)-5-(3-\{(1 S)-1-[\quad(S)-((2 S)-2-A z i d o-3-$ meth y l b t y y lamino ) - (tert butoxycarbonylmethylcarbamoyl)methyl]-1methylpropoxy -4-benzyloxyphenyl)-2-(4methoxyphenyl)-4,5-dihydrooxazole-4carboxylic acid methyl ester (52). Azide 46 (684 mg, $0.974 \mathrm{mmol}, 1$ equiv) was dissolved in $\mathrm{THF}(8 \mathrm{~mL})$ and water $(2 \mathrm{~mL})$ at room temperature. Trimethylphosphine () $(2.9 \mathrm{~mL}$,

$2.9 \mathrm{mmol}, 4$ equiv, $1 \mathrm{M}$ solution in THF) was added and the reaction was allowed to stir $4 \mathrm{~h}$. THF was removed under reduced pressure. Ethyl acetate and saturated sodium bicarbonate solution were added. The layers were separated and the aqueous layer was extracted twice more with ethyl acetate. The combined organic layers were washed once with brine and dried over anhydrous $\mathrm{Na}_{2} \mathrm{SO}_{4}$. Solvents were removed under reduced pressure and the crude product was loaded onto a silica gel column. The desired amine 52 (498 $\mathrm{mg}, 0.737 \mathrm{mmol}, 76 \%$ ) eluted using a gradient of $25 / 1$ to $5 / 1$ chloroform/methanol. $\mathrm{R}_{\mathrm{f}}=0.2$ (1/2 hexanes/ethyl acetate); IR (film) $3375 \mathrm{w}, 2976 \mathrm{w}$, 2935 w, 1743 s, 1674 m, 1645 m, 1609 m, 1511 s, 1506 s, 1455 w, 1367 w, 1305 w, 1257 s, 1223 m, 1170 s, 1082 w, 1026 w, 1008 w, 843 w, 741 w, 698 w; ${ }^{1}$ H NMR (500 MHz, $\left.\mathrm{CDCl}_{3}\right) \delta 7.97$ (d, J=9 Hz, 2H), 7.73 (7.81) (br, 1H), 7.37-7.32 (mult, 5H), 7.24-7.23 (mult, 1H), 7.04-7.02 (mult, 2H), 6.92 (d, J=9 Hz, 2H), 5.74 (d, J=7 Hz, 1H), 5.02 (5.05) $(\mathrm{s}, 2 \mathrm{H}), 4.72(\mathrm{~d}, \mathrm{~J}=7.5 \mathrm{~Hz}, 1 \mathrm{H}), 3.84$ (s, 3H), 3.83 (s, 3H), 3.64 (s, 2H), 1.91 (mult, 1H), 1.77 (mult, 1H), 1.39 (1.42) (s, 9H), 1.25 (1.69) (s, 3H), 0.93 (1.02) (t, J=7.5 Hz, 3H); ${ }^{13} \mathbf{C}$ NMR $\left(100 \mathrm{MHz}, \mathrm{CDCl}_{3}\right) \delta 173.2$ (173.0), 171.5, 168.9, 165.2, 162.6, 152.4, 144.1, 136.1, 132.2 (132.3), 130.4, 128.7, 128.6, 128.4 (128.3), 127.8, 121.0 (122.2), 121.2 (121.3), 119.2, 113.8, 113.5 (113.4), 86.4, 82.6, 81.6 (81.8), 70.9, 60.6, 55.4, 52.8, 41.7, 28.6 (29.5), 28.0, 19.9 (19.6), 8.1 (8.2); HRMS calculated for $\mathrm{C}_{37} \mathrm{H}_{45} \mathrm{~N}_{3} \mathrm{O}_{9}(\mathrm{M}+\mathrm{H})$ : 676.3228, found (MALDI): 676.3218. The chemical shifts in parentheses are the shifts for the minor diastereomer. 


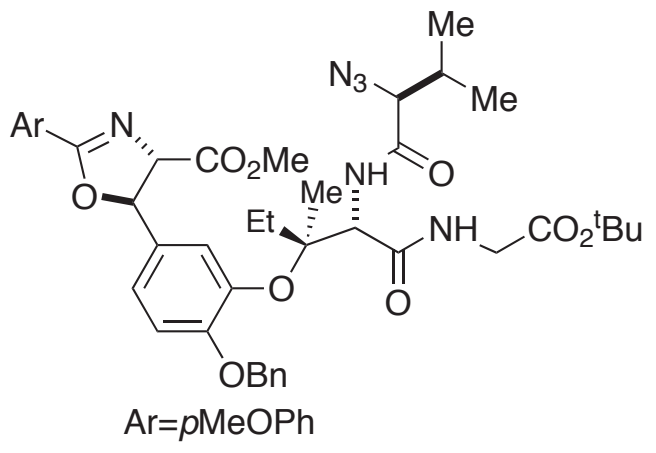

$(4 S, 5 R)-5-(3-\{(1 S)-1-[(S)-((2 S)-$ Azido-3methylbutyrylamino)-(tertbutoxycarbonylmethylcarbamoyl)methyl]-1methylpropoxy\}-4-benzyloxyphenyl)-2-(4methoxyphenyl)-4,5-dihydrooxazole-4carboxylic acid methyl ester (54). Amine 52 (490 $\mathrm{mg}, 0.724 \mathrm{mmol}, 1$ equiv) and azidovaline ${ }^{8} 53$ ( $157 \mathrm{mg}, 1.09 \mathrm{mmol}, 1.5$ equiv) were dissolved in acetonitrile $(7 \mathrm{~mL})$ at room temperature. BOP reagent $(642 \mathrm{mg}, 1.45$

mmol, 2 equiv) and diisopropylethylamine ( $460 \mu \mathrm{L}, 2.64 \mathrm{mmol}, 4$ equiv) were added and the reaction was stirred for $9 \mathrm{~h}$. The reaction was diluted with ethyl acetate and saturated sodium bicarbonate solution was added. The layers were separated and the aqueous layer was extracted with ethyl acetate. The combined organic layers were washed once with brine and dried over anhydrous $\mathrm{Na}_{2} \mathrm{SO}_{4}$. Solvents were removed under reduced pressure, and the crude product was loaded onto a silica gel column. The desired product, 54 (599 $\mathrm{mg}, 0.713 \mathrm{mmol}, 98 \%$ ) was obtained upon elution with $1 / 1$ to $1 / 2$ hexanes/ethyl acetate. $\mathrm{R}_{\mathrm{f}}=0.5(1 / 2$ hexanes/ethyl acetate); IR (film) $3373 \mathrm{w}, 2970 \mathrm{w}, 2930,2099 \mathrm{~m}, 1741 \mathrm{~s}$, 1672 s, 1609 m, 1512 s, 1455 w, 1368 w, 1257 s, 1222 m, 1170 s, 1127 w, 1082 w, 1026 m, 1008 w, 844 m, 742 w, 698 w; ${ }^{1} \mathbf{H}$ NMR $\left(500 \mathrm{MHz}, \mathrm{CDCl}_{3}\right) \delta 7.95(\mathrm{~d}, \mathrm{~J}=8.5 \mathrm{~Hz}, 2 \mathrm{H}$, 7.43-7.22 (mult, 7H), 7.03 (d, J=1.5 Hz, 1H), 7.07-6.94 (mult, 1H), 6.91 (d, J=8.5 Hz, $1 \mathrm{H}), 6.90(\mathrm{~d}, \mathrm{~J}=8.5 \mathrm{~Hz}, 2 \mathrm{H}), 5.73(5.72)(\mathrm{d}, \mathrm{J}=7.5 \mathrm{~Hz}, 1 \mathrm{H}), 5.21-5.02$ (mult, $1 \mathrm{H}), 5.11$ (5.12) (s, 2H), 4.79 (4.99) (d, J=9 (8) Hz, 1H), 4.70 (d, J=7.5 Hz, 1H), 3.83 (s, 3H), 3.81 (s, 3H), 3.67-3.62 (mult, $2 \mathrm{H}$ ), 2.32-2.25 (mult, 1H), 2.00-194 (1.70-1.64) (mult, $1 \mathrm{H}$ ), 1.78-1.71 (1.64-1.54) (mult, 1H), 1.35 (1.38) (s, 9H), 1.25 (1.17) (s, 3H), 0.99 (0.98) (d, $\mathrm{J}=7 \mathrm{~Hz}, 3 \mathrm{H}), 1.01(0.88)(\mathrm{t}, \mathrm{J}=6.5 \mathrm{~Hz}, 3 \mathrm{H}), 0.86(\mathrm{~d}, \mathrm{~J}=7 \mathrm{~Hz}, 3 \mathrm{H}) ;{ }^{13} \mathbf{C} \mathbf{N M R}(125.5 \mathrm{MHz}$, $\left.\mathrm{CDCl}_{3}\right) \delta 171.4,169.3,169.2$ (168.9), 168.5 (168.2), 165.2, 162.6, 151.7, 143.6 (143.5), 136.1 (135.7), 132.4 (132.2), 130.4, 128.9, 128.7, 128.5, 128.1, 127.4, 123.4, 122.0, $120.9,119.9$ (119.1), 113.8, 113.3, 85.4 (86.1), 82.5, 81.7 (81.8), 76.7 (76.6), 71.0 (70.5), 58.2 (58.9), 55.4, 52.8, 41.8 (42.1), 31.8 (31.9), 30.1 (28.7), 27.9 (28.0), 20.1 (20.5), 19.5, 17.1 (17.2), 8.4 (8.3); HRMS calculated for $\mathrm{C}_{42} \mathrm{H}_{52} \mathrm{~N}_{6} \mathrm{O}_{10}(\mathrm{M}+\mathrm{H})$ : 801.3817, found (MALDI): 801.3794. The chemical shifts in parentheses are the shifts for the minor diastereomer.

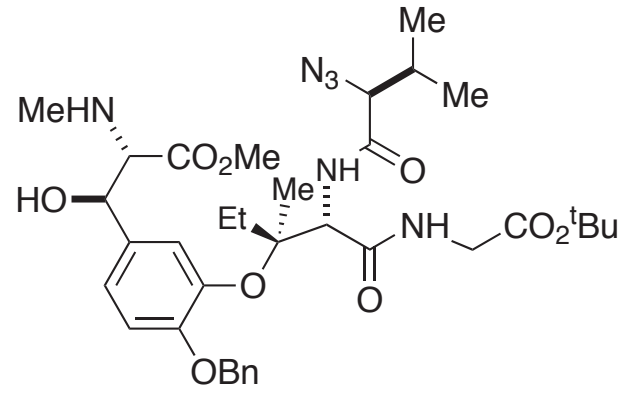

$(2 S, 3 R)-3-(3-\{(1 S)-1-[(S)-((2 S)-2-A z i d o-3-$ methylbutyrylamino)-(tertbutoxycarbonylmethylcarbamoyl)methyl]-1methylpropoxy\}-4-benzyloxyphenyl)-3hydroxy-2-methylaminopropionic acid methyl ester (55). Oxazoline 54 (579 mg, $0.687 \mathrm{mmol}$, 1 equiv) was dissolved in dichloromethane (12 $\mathrm{mL}$ ) at room temperature. Methyl trifluoromethanesulfonate (freshly distilled from

\footnotetext{
${ }^{8}$ Hackeng, T. M.; Griffin, J. H.; Dawson, P. E. Proc. Natl. Acad. Sci. USA 1999, 96, 10068-10073.
} 
calcium hydride) (95 $\mu \mathrm{L}, 0.84 \mathrm{mmol}, 1.2$ equiv) was added and the reaction was stirred for $2 \mathrm{~h}$. Toluene was added and the reaction mixture was azeotroped and dried under high vacuum $(0.5 \mathrm{mmHg})$. The residue was dissolved in dichloromethane $(20 \mathrm{~mL})$ at 0 ${ }^{\circ} \mathrm{C}$. Saturated sodium bicarbonate solution $(20 \mathrm{~mL})$ and sodium borohydride $(51.5 \mathrm{mg}$, $1.36 \mathrm{mmol}, 2$ equiv) were added at once with vigorous stirring. After $10 \mathrm{~min}$, the layers were separated, and the aqueous layer was extracted twice more with dichloromethane. The combined organic layers were dried over anhydrous $\mathrm{Na}_{2} \mathrm{SO}_{4}$. The solvent was removed under reduced pressure and the crude product was purified using silica gel column chromatography. The desired $(4 S, 5 R)-5-(3-\{(1 S)-1-[(S)-((2 S)-2$-azido-3methylbutyrylamino)-(tert-butoxycarbonylmethylcarbamoyl)methyl]-1-methylpropoxy\}4-benzyloxyphenyl)-2-(4-methoxyphenyl)-3-methyloxazolidine-4-carboxylic acid methyl ester $(549 \mathrm{mg}, 0.672 \mathrm{mmol}$ ) was obtained upon elution with $2 / 1$ to $1 / 1$ hexanes/ethyl acetate. $\mathrm{R}_{\mathrm{f}}=0.8(1 / 2$ hexanes/ethyl acetate $)$, followed by the recovered starting material 54 (37.7 mg, $0.0471 \mathrm{mmol}, 7 \%)$.

$(4 S, 5 R)-5-(3-\{(1 S)-1-[(S)-((2 S)-2-A z i d o-3-m e t h y l b u t y r y l a m i n o)-(t e r t-$ butoxycarbonylmethylcarbamoyl)methyl]-1-methylpropoxy\}-4-benzyloxyphenyl)-2-(4methoxyphenyl)-3-methyloxazolidine-4-carboxylic acid methyl ester (549 mg, 0.672 $\mathrm{mmol})$ was dissolved in THF $(20 \mathrm{~mL})$ at room temperature. Oxalic acid $(0.1 \mathrm{~N}$ solution in water) $(16 \mathrm{~mL})$ was added and the reaction was stirred for $9 \mathrm{~h}$. THF was removed under reduced pressure, and chloroform and saturated sodium bicarbonate solution were added. The layers were separated and the aqueous layer was extracted twice more with chloroform. The combined organic layers were dried over anhydrous $\mathrm{Na}_{2} \mathrm{SO}_{4}$. The solvent was removed under reduced pressure and the crude products were purified using silica gel column chromatography. Using 1/1 hexanes/ethyl acetate, $p$ methoxybenzaldehyde and the over-reduced product that had formed in the methylation step eluted. The desired product 55 (334 mg, $0.470 \mathrm{mmol}, 68 \%$ over 2 steps) was obtained upon elution with a gradient of 1/4 hexanes/ethyl acetate, $100 \%$ ethyl acetate and 20/1 chloroform/methanol. $\mathrm{R}_{\mathrm{f}}=0.2$ (1/2 hexanes/ethyl acetate); ${ }^{1} \mathbf{H}$ NMR $(500 \mathrm{MHz}$, $\mathrm{CD}_{3} \mathrm{OD}$ ) $\delta$ 7.50-7.46 (mult, 2H), 7.32-7.41 (mult, 3H), 7.16 (7.12) (d, J=2 Hz, 1H), 7.017.07 (mult, 1H), 6.98 (6.96) (d, J=2 Hz, 1H), 5.15 (s, 2H), 4.81 (4.88) (s, 1H), 4.65 (d, $\mathrm{J}=7 \mathrm{~Hz}, 1 \mathrm{H}), 3.74-3.41$ (mult, $2 \mathrm{H}$ ), 3.35 (d, J=7.5 Hz, 1H), 2.31 (s, 3H), 2.20-2.13 (mult, 1H), 2.09-2.04 (1.81-1.76) (mult, 1H), 1.87-1.83 (1.72-1.67) (mult, 1H), 1.40 (1.44) (s, $9 \mathrm{H}), 1.34$ (1.35) (s, 3H), $0.96(1.01)(\mathrm{t}, \mathrm{J}=6.5(7.5) \mathrm{Hz}, 3 \mathrm{H}), 0.96$ (d, J=6.5 Hz, 3H), 0.92 $(\mathrm{d}, \mathrm{J}=6.5 \mathrm{~Hz}, 3 \mathrm{H})$. The chemical shifts in parentheses are the shifts for the minor diastereomer.

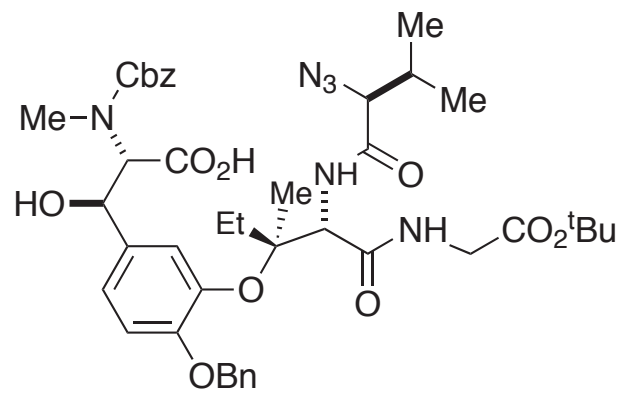

$(2 S, 3 R)-3-(3-\{(1 S)-1-[(S)-((2 S)-2-A z i d o-3-$ methylbutyrylamino)-(tertbutoxycarbonylmethylcarbamoyl)methyl]-1methylpropoxy\}-4-benzyloxyphenyl)-2(benzyloxycarbonylmethylamino)-3hydroxypropionic acid (56). Methyl ester 55 (71.2 $\mathrm{mg}, 0.104 \mathrm{mmol}, 1$ equiv) was dissolved in THF $(6 \mathrm{~mL})$ at $0{ }^{\circ} \mathrm{C}$. Lithium hydroxide $(3 \mathrm{~mL}$, $0.3 \mathrm{mmol}, 3$ equiv, $0.1 \mathrm{~N}$ solution in water) was added and the reaction was stirred for $1.5 \mathrm{~h}$ at $0{ }^{\circ} \mathrm{C}$. At this point, TLC showed complete 
methyl ester hydrolysis, so benzyl chloroformate ( $45 \mu \mathrm{L}, 0.32 \mathrm{mmol}, 3$ equiv) was added and the reaction was stirred for one additional hour. The reaction was diluted with chloroform and $\mathrm{pH} 2.5$ aqueous phosphate buffer was added. The layers were separated and the aqueous layer was extracted twice more with chloroform. The combined organic layers were dried over anhydrous $\mathrm{Na}_{2} \mathrm{SO}_{4}$. The solvent was removed under reduced pressure and the crude product was purified using silica gel column chromatography. Excess benzyl chloroformate was eluted using 1/1 hexanes/ethyl acetate, and the desired product $56(59.4 \mathrm{mg}, 0.0725 \mathrm{mmol}, 70 \%)$ was obtained upon elution with a gradient of 25/1 to 4/1 chloroform/methanol. $\mathrm{R}_{\mathrm{f}}=0.6$ (5/1 chloroform/methanol); IR (film) $3374 \mathrm{br}$, 2971 w, 2935 w, 2099 m, 1740 w, 1669 s, 1507 m, 1454 w, 1392 m, 1368 m, 1304 w, 1258 m, 1221 m, 1154 s, 1050 m, 1026 s, 1005 s, 848 w, 824 w, 766 w, 699 w; ${ }^{1}$ H NMR (500 MHz, DMSO-d ${ }_{6}, 85^{\circ} \mathrm{C}$ ) $\delta 7.92$ (br, 1H), 7.49-7.10 (mult, 8H), 6.93 (br, 1H), 5.08 (5.13) (s, 2H), 4.93 (4.89) (s, 1H), 4.79 (br, 1H), 3.76-3.54 (mult, 5H), 2.85 (2.86) (s, 3H), 2.03-2.12 (mult, 1H), 1.93-1.84 (mult, 1H), 1.78-1.69 (mult, 1H), 1.35 (1.38) (s, 9H), 1.20 (1.27) (s, 3H), 0.95-0.81 (mult, 9H); ${ }^{13} \mathbf{C}$ NMR (125.5 MHz, DMSO-d 6 , $85{ }^{\circ} \mathrm{C}$ ) ठ $168.7,168.3,168.0,164.7,162.9,155.3,142.5,137.5,136.8,127.8,127.7,127.3$, $127.1,126.5,125.8,121.9,113.2,84.1,83.7,80.1,70.2,68.0,57.7,30.3,29.7,28.4,27.5$, 27.4, 27.2, 27.1, 19.2, 18.4, 17.7, 7.8, 7.4; HRMS calculated for $\mathrm{C}_{42} \mathrm{H}_{54} \mathrm{~N}_{6} \mathrm{O}_{11}(\mathrm{M}+\mathrm{Na})$ : 841.3743, found (MALDI): 841.3749. The chemical shifts in parentheses are the shifts for the minor diastereomer.

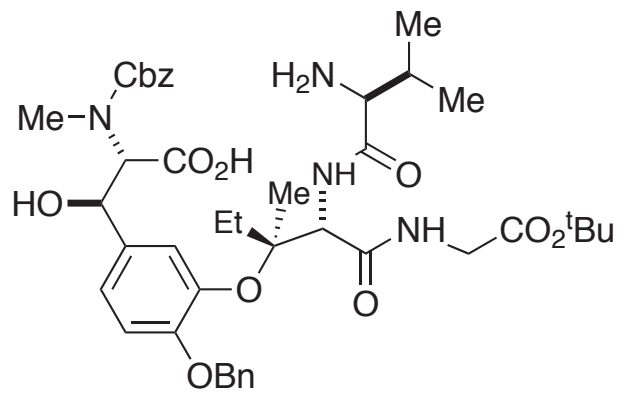

\section{S,3R)-3-(3-\{(1S)-1-[(S)-((2S)-2-Azido-3- methylbutyrylamino)-(tert- butoxycarbonylmethylcarbamoyl)methyl]-1- methylpropoxy\}-4-benzyloxyphenyl)-2- (benzyloxycarbonylmethylamino)-3-} hydroxypropionic acid (57). Azide 56 (49.9 $\mathrm{mg}, 0.0609 \mathrm{mmol}, 1$ equiv) was dissolved in THF $(0.6 \mathrm{~mL})$ and water $(0.15 \mathrm{~mL})$ at room temperature. Trimethylphosphine $(240 \mu \mathrm{L}, 0.240$

mmol, 4 equiv, $1 \mathrm{M}$ solution in THF) was added and the reaction was stirred for $10 \mathrm{~h}$. Solvents were removed under reduced pressure, and the remaining reaction mixture was loaded directly onto a silica gel column. Using a gradient of $25 / 1$ to $5 / 1$ chloroform/methanol, 57 (34.9 $\mathrm{mg}, 0.0440 \mathrm{mmol}, 72 \%)$ was obtained. The two diastereomers showed different $\mathrm{R}_{\mathrm{f}}$ values on TLC but could not be separated. $\mathrm{R}_{\mathrm{F}}=0.5$ (major isomer) (5/1 chloroform/methanol), $\mathrm{R}_{\mathrm{f}}=0.4$ (minor isomer) $(5 / 1$ chloroform/methanol); IR (film) 3363 br,s, 2976 m, 2935 m, 1742 m, 1674 s, 1606 m, $1505 \mathrm{~m}, 1455 \mathrm{w}, 1386 \mathrm{~m}, 1367 \mathrm{~m}, 1311 \mathrm{w}, 1258 \mathrm{~m}, 1222 \mathrm{~m}, 1155 \mathrm{~s}, 1122 \mathrm{w}, 1049 \mathrm{w}$, 1026 m, 1003 m, 947 w, 848 w, 824 w, 752 m, 698 m; ${ }^{1} \mathbf{H}$ NMR (500 MHz, DMSO-d 6 , $\left.80{ }^{\circ} \mathrm{C}\right) \delta 7.87$ (br, 1H), 7.44-7.20 (mult, 8H), 6.95 (br, 1H), $5.01(5.13)(\mathrm{s}, 2 \mathrm{H}), 4.97$ (s, 1H), 4.66 (br, 1H), 3.67-3.55 (mult, 2H), 3.37 (d, J=4 Hz, 1H), 2.73 (2.91) (s, 3H), 2.012.04 (mult, 1H), 1.83-1.74 (mult, 2H), 1.36 (1.38) (s, 9H), 1.19 (1.26) (s, 3H), 0.93 (d, $\mathrm{J}=7 \mathrm{~Hz}, 3 \mathrm{H}), 0.90(0.80)(\mathrm{t}, \mathrm{J}=7 \mathrm{~Hz}, 3 \mathrm{H}), 0.85$ (d, J=6.5 Hz, 3H); ${ }^{13} \mathbf{C}$ NMR $(125.5 \mathrm{MHz}$, DMSO-d $\left.{ }_{6}, 80{ }^{\circ} \mathrm{C}\right) \delta 172.0,171.4,169.0,168.1,150.9,142.8,136.8,135.5,127.8,127.2$, 126.6, 125.9, 122.4, 121.8, 113.1, 83.2, 80.1, 72.1, 70.1, 65.6, 63.7, 58.7, 30.6, 28.1, 27.5, 27.4, 27.2, 19.2, 18.6, 16.6, 7.4; HRMS calculated for $\mathrm{C}_{42} \mathrm{H}_{56} \mathrm{~N}_{4} \mathrm{O}_{11}(\mathrm{M}+\mathrm{Na}): 815.3838$, 
found (MALDI): 815.3829. The chemical shifts in parentheses are the shifts for the minor diastereomer.

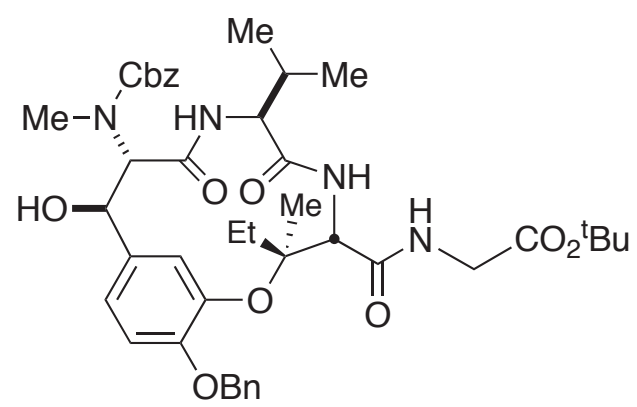

\section{(benzyloxycarbonylmethylamino)-3-ethyl-11-} hydroxyl-7-isopropyl-3-methyl-6,9-dioxo-2oxa-5,8-diaza-bicyclo[10.3.1]hexadeca[(16),12,14-triene-4-carbonyl]amino\}acetic acid tert-butyl ester (58). 7-Azabenzotriazol1-yloxy-tris(pyrrolidino)phosphonium hexafluorophosphate (PyAOP) $(21.4 \mathrm{mg}, 0.0410$ mmol, 3 equiv), DMF $(5 \mathrm{~mL})$ and diisopropylethylamine ( $12 \mu \mathrm{L}, 0.069 \mathrm{mmol}, 5$ equiv) were charged in a flame dried flask at room temperature. Amino acid $57(10.7 \mathrm{mg}, 0.0135 \mathrm{mmol})$ in DMF $(0.3 \mathrm{~mL})$ was added slowly using a syringe pump $(0.3 \mathrm{~mL} / \mathrm{h})$. When the addition was complete, the reaction was stirred for another $1 \mathrm{~h}$ and then diluted with ether. Water was added and the layers were separated. The aqueous layer was extracted twice with ether. The combined organic layers were dried over anhydrous $\mathrm{Na}_{2} \mathrm{SO}_{4}$. After the removal of the solvent, the crude material was purified using silica gel column chromatography. Using a gradient of $2 / 1$ to $1 / 2$ hexanes/ethyl acetate, $58(6.5 \mathrm{mg}, 0.0084 \mathrm{mmol}, 69 \%)$ was obtained. Some starting amino acid $57(1.0 \mathrm{mg}, 0.0013 \mathrm{mmol}, 9 \%)$ was recovered from the rinse of the syringe. $R_{\mathrm{f}}=0.4(1 / 2$ hexanes/ethyl acetate); $\mathbf{I R}$ (film) $3406 \mathrm{w}, 3333 \mathrm{~m}, 2956 \mathrm{~m}, 1925 \mathrm{~m}$, $2848 \mathrm{~m}, 1793 \mathrm{w}, 1661 \mathrm{~s}, 1525 \mathrm{~m}, 1505 \mathrm{~m}, 1455 \mathrm{~m}, 1367 \mathrm{~m}, 1260 \mathrm{~m}, 1223 \mathrm{~m}, 1155 \mathrm{~s}$, 1124 w, 1075 w, 998 w, 959 w, 845 w, 809 w, 734 w, 696 w; ${ }^{1} \mathbf{H}$ NMR $(500$ MHz, DMSO-d $\left._{6}, 70{ }^{\circ} \mathrm{C}\right) \delta 7.99(\mathrm{t}, \mathrm{J}=6 \mathrm{~Hz}, 1 \mathrm{H}), 7.94(\mathrm{~d}, \mathrm{~J}=6.5 \mathrm{~Hz}, 1 \mathrm{H}), 7.46-7.30$ (mult, $7 \mathrm{H}$ ), 7.12 (d, J=8.5 Hz, 1H), 6.94 (d, J=8.5 Hz, 1H), 5.48 (br, 1H), 5.16-5.01 (mult, 3H), 4.91 $(\mathrm{d}, \mathrm{J}=10 \mathrm{~Hz}, 1 \mathrm{H}), 4.62(\mathrm{~d}, \mathrm{~J}=10 \mathrm{~Hz}, 1 \mathrm{H}), 4.38$ (d, J=8.5 Hz, 1H), 3.85 (dd, J=6.5, $17 \mathrm{~Hz}$, $1 \mathrm{H}$ ), 3.76 (dd, J=5, $17 \mathrm{~Hz}, 1 \mathrm{H}$ ), 2.13-2.08 (mult, 1H), 2.02-1.96 (mult, 1H), 1.96-1.88 (mult, 1H), 1.42 (s, 9H), $1.11(\mathrm{~s}, 3 \mathrm{H}), 0.95$ (t, J=8 Hz, 3H), 0.75 (d, J=7 Hz, 3H), 0.73 (d, $\mathrm{J}=7 \mathrm{~Hz}, 3 \mathrm{H}) ;{ }^{13} \mathbf{C}$ NMR $\left(125.5 \mathrm{MHz}, \mathrm{DMSO}_{6}, 70{ }^{\circ} \mathrm{C}\right) \delta 170.5,170.0,168.8,168.4$, $168.1,168.0,155.7,151.7,151.5,143.7,143.6,136.9,136.7,134.7,134.6,127.8,127.2$, $126.8,123.7,123.6,123.2,121.9,114.7,84.0,83.8,80.3,70.4,70.1,69.8,69.4,68.7$, $68.5,65.7,63.9,60.1,59.8,58.9,58.6,58.1,42.0,41.6,30.7,29.7,27.6,27.4,27.3,27.1$, $19.3,18.6,18.6,17.4,17.4,7.9,7.8$; HRMS calculated for $\mathrm{C}_{42} \mathrm{H}_{54} \mathrm{~N}_{4} \mathrm{O}_{10}(\mathrm{M}+\mathrm{Na})$ : 797.3732, found (MALDI): 797.3749.

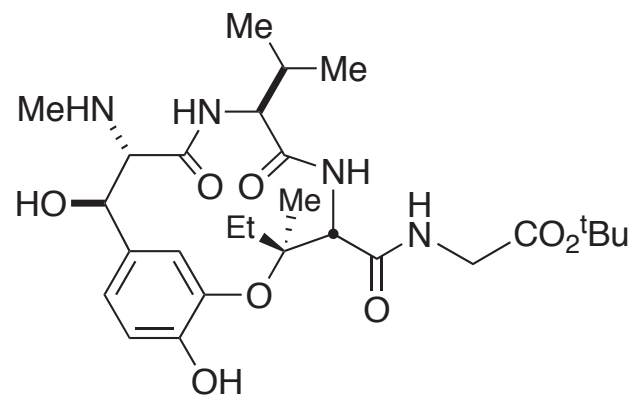

C2-epi-Ustiloxin D tert-butyl ester (74). Macrocycle 58 (10.2 mg, $0.0132 \mathrm{mmol}, 1$ equiv) was dissolved in methanol $(1 \mathrm{~mL})$ at room temperature. $5 \%$ Palladium on carbon $(5.0 \mathrm{mg}$, $50 \%$ weight of substrate, purchased from Acros) was added and a hydrogen balloon was set. After $5.5 \mathrm{~h}$, hydrogen was released and the reaction mixture was concentrated. The crude material was purified by directly loading onto a silica gel column. Using a gradient of $20 / 1$ to $10 / 1$ chloroform/methanol, C2-epi- 
Ustiloxin D tert-butyl ester $74(5.8 \mathrm{mg}, 0.011 \mathrm{mmol}, 80 \%)$ was obtained. $\mathrm{R}_{\mathrm{f}}=0.2(5 / 1$ chloroform/methanol); IR (film) $3300 \mathrm{~m}, 2975 \mathrm{~m}, 2935 \mathrm{~m}, 1747 \mathrm{~m}, 1652 \mathrm{~s}, 1538 \mathrm{~m}$, 1520 s, 1456 w, 1388 w, 1369 m, 1286 m, 1224 m, 1157 m, 1133 w, 1116 w, 1063 w, $961 \mathrm{w}, 887$ w, 844 w, 755 m; ${ }^{1} \mathbf{H}$ NMR (500 MHz, CD $\left.\mathrm{OD}\right) \delta 7.16$ (dd, J=2, $\left.8 \mathrm{~Hz}, 1 \mathrm{H}\right)$, 7.03 (br, 1H), 6.90 (d, J=8.5 Hz, 1H), 4.65 (s, 1H), 4.46 (d, J=9 Hz, 1H), 3.97 (d, J=7.5 $\mathrm{Hz}, 1 \mathrm{H}), 3.61$ (d, J=18 Hz, 1H), 3.83 (d, J=18 Hz, 1H), 3.17 (d, J=9 Hz, 1H), 2.33 (S, $3 \mathrm{H}$ ), 2.31-2.24 (mult, 1H), 2.16-2.09 (mult, 1H), 2.04-1.96 (mult, 1H), 1.45 (s, 9H), 1.38 $(\mathrm{s}, 3 \mathrm{H}), 1.41(\mathrm{t}, \mathrm{J}=7.5 \mathrm{~Hz}, 3 \mathrm{H}), 0.82(\mathrm{~d}, \mathrm{~J}=7 \mathrm{~Hz}, 3 \mathrm{H}), 0.81(\mathrm{~d}, \mathrm{~J}=7.5 \mathrm{~Hz}, 3 \mathrm{H}) ;{ }^{13} \mathbf{C}$ NMR (125.5 MHz, $\left.\mathrm{CD}_{3} \mathrm{OD}\right) \delta 175.2,171.7,171.4,170.0,152.1,143.6,134.0,124.6,123.2$, 118.6, 85.7, 82.8, 75.5, 73.3, 61.2, 61.0, 42.9, 34.5, 31.0, 29.6, 28.3, 22.8, 19.4, 18.4, 8.5; HRMS calculated for $\mathrm{C}_{27} \mathrm{H}_{42} \mathrm{~N}_{4} \mathrm{O}_{8}(\mathrm{M}+\mathrm{Na}): 573.2895$, found (MALDI): 573.2898.

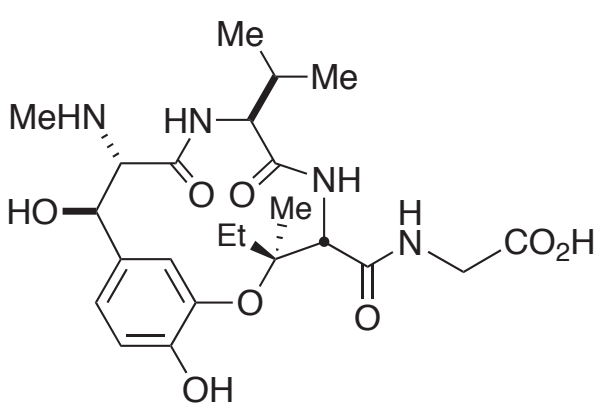

C2-epi-Ustiloxin D (59). C2-epi-Ustiloxin D tert-butyl ester 74 (3.4 $\mathrm{mg}, 0.0062 \mathrm{mmol}, 1$ equiv) was dissolved in THF $(0.5 \mathrm{~mL})$ at room temperature. Lithium hydroxide $(0.5 \mathrm{~mL}, 0.05$ mmol, 9 equiv, $1 \mathrm{~N}$ solution in water) was added and the reaction was stirred for $8 \mathrm{~h}$. The reaction mixture was acidified by the addition of aqueous $1 \mathrm{~N} \mathrm{HCl}$ (about 3 drops) and the solvent was removed under reduced pressure. The crude product was purified using a Sep-Pak C18. The Sep-Pak column was activated prior to use by pre-treating with $5 \mathrm{~mL}$ methanol followed by $15 \mathrm{~mL}$ water. The crude compound was loaded using water. Water $(3 \mathrm{~mL})$ was run to elute all the inorganic salts. The desired compound C2-epi-Ustiloxin D 59 (2.6 mg, $0.0053 \mathrm{mmol}, 85 \%)$ was eluted using $1 / 1$ water/methanol $(6 \mathrm{~mL}) . \quad \mathrm{R}_{\mathrm{f}}=0.5$ (1/2 chloroform/methanol); $[\alpha]_{\mathrm{D}}{ }^{23}-90.8^{\circ}$ (c 0.33 , $\mathrm{H}_{2} \mathrm{O}$ ); IR (KBr) $3411 \mathrm{~s}, 2966$ w, 2935 w, 1657 s, 1610 m, 1517 m, 1463 w, 1385 m, 1287 m, 1220 w, 1155 w, 1113 m, 1073 s, 962 w, 794 w, 783 w; ${ }^{1} \mathbf{H}$ NMR (500 MHz, D 20$) \delta$ $6.98(\mathrm{dd}, \mathrm{J}=2,8.5 \mathrm{~Hz}, 1 \mathrm{H}), 6.88$ (d, J=8.5 Hz, 1H), 6.67 (br, 1H), 4.63 (s, 1H), 4.53 (d, $\mathrm{J}=9.5 \mathrm{~Hz}, 1 \mathrm{H}), 3.84(\mathrm{~d}, \mathrm{~J}=9 \mathrm{~Hz}, 1 \mathrm{H}), 3.69$ (d, J=9.5 Hz, 1H), 3,56 (s, 2H), 2.54 (s, 3H), 2.10 (dq, J=7.5, $21.5 \mathrm{~Hz}, 1 \mathrm{H}), 1.72$ (dq, J=7.5, $21.5 \mathrm{~Hz}, 1 \mathrm{H}$ ), 1.60 (mult, 1H), 1.31 (s, $3 \mathrm{H}), 0.99(\mathrm{t}, \mathrm{J}=7.5 \mathrm{~Hz}, 3 \mathrm{H}), 0.63(\mathrm{~d}, \mathrm{~J}=6.5 \mathrm{~Hz}, 3 \mathrm{H}), 0.53(\mathrm{~d}, \mathrm{~J}=7 \mathrm{~Hz}, 3 \mathrm{H}) ;{ }^{13} \mathbf{C} \mathbf{~ N M R}$ $\left(125.5 \mathrm{MHz}, \mathrm{D}_{2} \mathrm{O}\right) \delta 175.9,170.5,170.4,165.7,150.8,142.1,130.1,123.1,122.2,118.6$, 72.9, 68.7, 68.3, 59.8, 59.6, 43.4, 32.0, 28.4, 23.1, 18.1, 17.7, 6.8; HRMS calculated for $\mathrm{C}_{23} \mathrm{H}_{34} \mathrm{~N}_{4} \mathrm{O}_{8}(\mathrm{M}+\mathrm{H}): 495.2449$, found (MALDI): 495.2453.

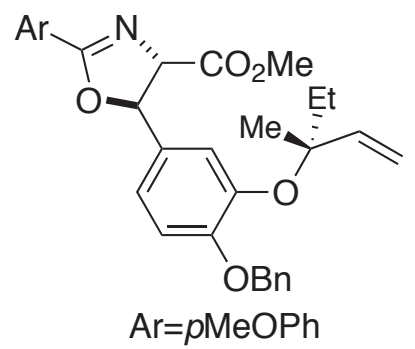

$(4 S, 5 R)-5-[3-(B e n z y l o x y-4-)-1-(1 R)-e t h y l-1-$ methylallyloxy)phenyl]-2-(4-methoxyphenyl)-4,5dihydrooxazole-4-carboxylic acid methyl ester (41a).

Phenol 40 (3.51 g, $8.09 \mathrm{mmol}$, 1 equiv) was dissolved in dichloromethane (degassed by bubbling argon for $20 \mathrm{~min}$ ) (30 $\mathrm{mL})$ at room temperature. $(1 S, 2 S)-1,2$ - b is $(2$ diphenylphosphinobenzoyl)aminocyclohexane 60 (335.4 mg, $0.486 \mathrm{mmol}, 0.06$ equiv) and tris(dibenzylideneacetone)dipalladium(0)-chloroform adduct $(167.7 \mathrm{mg}, 0.162 \mathrm{mmol}, 0.02$ equiv) were added. Carbonic acid-1-ethyl-1-methylallyl ester methyl ester 16 (4.0 mL, $24.3 \mathrm{mmol}, 3$ equiv) 
was added dropwise over $1 \mathrm{~min}$. The reaction turned yellow. When $\mathbf{1 6}$ was fully consumed, the reaction color changed to orange. Subsequent aliquots of $16(333 \mu \mathrm{L}$, $2.11 \mathrm{mmol}, 0.25$ equiv) were added every $10 \mathrm{~min}$ over $40 \mathrm{~min}$ as this color change to orange was observed. The catalyst had ceased to react after $1 \mathrm{~h}$ and the crude mixture was loaded directly to a silica gel column. A gradient of 10/1 to 5/1 hexanes/ethyl acetate eluted excess 16 and dibenzylideneacetone. The product 41a, $(3.14 \mathrm{~g}, 6.09 \mathrm{mmol}$, $70 \%, 26 \% d e$ ) eluted with $2 / 1$ hexanes/ethyl acetate and recovered starting material 40 (1.07 g, $2.47 \mathrm{mmol}, 30 \%$ ) was obtained upon elution with $1 / 2$ hexanes/ethyl acetate. $\mathrm{R}_{\mathrm{f}}=0.4$ (2/1 hexanes/ethyl acetate); $\mathrm{Rt}=31.6 \mathrm{~min}$ (minor isomer 41b), $37.4 \mathrm{~min}$ (major isomer 41a), Chiralpak AD (0.9 mL/min, 80\% ethanol/hexane); IR (film) $2937 \mathrm{w}, 2358$ w, $1738 \mathrm{~m}, 1640 \mathrm{~m}, 1608 \mathrm{~m}, 1511 \mathrm{~m}, 1453 \mathrm{w}, 1371 \mathrm{w}, 1306 \mathrm{w}, 1255 \mathrm{~s}, 1223 \mathrm{~m}, 1169 \mathrm{~s}$, 1132 m, 1080 m, 1024 m, 921 w, 841 w, 738 w, 696 w; ${ }^{1} \mathbf{H}$ NMR (400 MHz, CDCl 3 ) $\delta$ 7.97 (d J=9 Hz, 2H), 7.42 (d J=7 Hz, 2H), 7.35 (t J=8 Hz, 2H), 7.29 (d J=7 Hz, 1H), 7.06 $(\mathrm{d} \mathrm{J}=2 \mathrm{~Hz}, 1 \mathrm{H}), 6.91(\mathrm{~d} \mathrm{~J}=9 \mathrm{~Hz}, 2 \mathrm{H}), 6.95-6.85(\mathrm{~m}, 2 \mathrm{H}), 6.02$ (dd J=11, $18 \mathrm{~Hz}, 1 \mathrm{H}), 5.72$ $(\mathrm{d} \mathrm{J}=7 \mathrm{~Hz}, 1 \mathrm{H}), 5.06(\mathrm{~s}, 2 \mathrm{H}), 5.03(\mathrm{~d} \mathrm{~J}=11 \mathrm{~Hz}, 1 \mathrm{H}), 5.03(\mathrm{~d} \mathrm{~J}=12 \mathrm{~Hz}, 1 \mathrm{H}), 4.71(\mathrm{~d} J=7$ $\mathrm{Hz}, 1 \mathrm{H}), 3.83$ (s, 3H), $3.81(\mathrm{~s}, 3 \mathrm{H}), 1.86-1.68(\mathrm{~m}, 2 \mathrm{H}), 1.33$ (s, 3H), 0.92 (t J=7 Hz, 3H); ${ }^{13}$ C NMR $\left(100 \mathrm{MHz}, \mathrm{CDCl}_{3}\right) \delta 171.5,165.2,162.5,152.1,146.2,143.0,137.1,132.1$, $130.4,128.4,127.7,127.2,120.3,120.1,119.3,114.8,114.6,113.6,83.7,82.8,76.7$, 71.0, 55.3, 52.7, 34.2, 21.0, 8.3; HRMS calculated for $\mathrm{C}_{31} \mathrm{H}_{33} \mathrm{NO}_{6}(\mathrm{M}+\mathrm{H}): 516.2381$, found (FAB): 516.2356 .

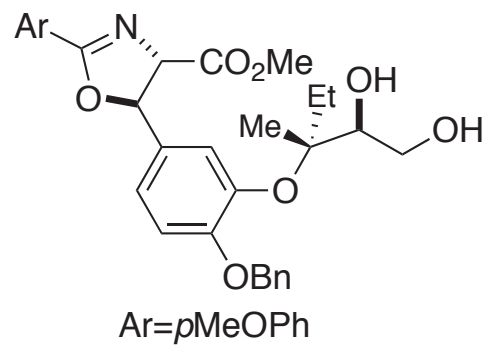

$(4 S, 5 R)-5-[4-B e n z y l o x y-3-((1 R, 2 S)-1-e t h y l-2,3-$ dihydroxy-1-methylpropoxy)phenyl-4,5dihydrooxazole-4-carboxylic acid methyl ester (42a). Terminal alkene $41 \mathrm{a}$ (4.15 g, $8.05 \mathrm{mmol}$, 1 equiv), potassium carbonate $(1.11 \mathrm{~g}, 8.05 \mathrm{mmol}, 1$ equiv), sodium bicarbonate (1.35 g, $16.1 \mathrm{mmol}, 2$ equiv), potassium ferricyanide(III) (7.95 g, $24.2 \mathrm{mmol}, 3$ equiv) were charged in a flask. (DHQD) ${ }_{2} \mathrm{PYR}(1.42 \mathrm{mg}, 1.61$

mmol, 0.2 equiv) was added as a solution in tert-butyl alcohol $(41 \mathrm{~mL})$, followed by potassium osmate dihydrate $(148.3 \mathrm{mg}, 0.402 \mathrm{mmol}, 0.05$ equiv) in water $(41 \mathrm{~mL})$. The reaction was stirred at room temperature for 4.5 days. Sodium sulfite $(12.07 \mathrm{~g}, 95.7$ mmol, 12 equiv) was added and the reaction was stirred for $1 \mathrm{~h}$ before diluting the mixture with water $(250 \mathrm{~mL})$. The aqueous layer was extracted with ethyl acetate $(3 \times 150 \mathrm{~mL})$. The organic layers were combined and dried over anhydrous $\mathrm{Na}_{2} \mathrm{SO}_{4}$. After removal of the solvent under reduced pressure, the crude yellow foam was purified using silica gel column chromatography. Recovered starting material 41a $(708.3 \mathrm{mg}$, $1.37 \mathrm{mmol}, 17 \%$ ) eluted using a gradient of $2 / 1$ to $1 / 1$ hexanes/ethyl acetate. The desired product 42a $(3.18 \mathrm{~g}, 5.79 \mathrm{mmol}, 72 \%, 66 \% \mathrm{de})$ was obtained by elution with a gradient of $1 / 2$ to $1 / 10$ hexanes/ethyl acetate to $100 \%$ ethyl acetate. $R_{\mathrm{f}}=0.3(1 / 2$ hexanes/ethyl acetate); IR (film) $3460 \mathrm{w}, 2940 \mathrm{w}, 1738 \mathrm{~m}, 1634 \mathrm{~m}, 1609 \mathrm{~m}, 1512 \mathrm{~s}, 1258 \mathrm{~s}, 1171 \mathrm{~s}$, 1132 m, 1083 m, 1026 m, 842 w, $742 \mathrm{w}, 698 \mathrm{w} ;{ }^{1} \mathbf{H}$ NMR $\left(400 \mathrm{MHz}, \mathrm{CDCl}_{3}\right) \delta 7.96$ (d $\mathrm{J}=9 \mathrm{~Hz}, 2 \mathrm{H}), 7.42-7.29(\mathrm{~m}, 5 \mathrm{H}), 7.06-6.88(\mathrm{~m}, 3 \mathrm{H}), 6.91(\mathrm{~d} \mathrm{~J}=9 \mathrm{~Hz}, 2 \mathrm{H}), 5.74(\mathrm{~d} \mathrm{~J}=6 \mathrm{~Hz}$, 1H), 5.06 (s, 3H), 4.72 (d J=8 Hz, 1H), 3.83 (s, 3H), 3.82 (s, 3H), $3.66(\mathrm{~d} \mathrm{~J}=8 \mathrm{~Hz}, 2 \mathrm{H})$, 2.46 (2.39) (d J=6 Hz, 1H), 1.91-1.78 (m, 1H), 1.62-1.57 (1.51-1.46) (m, 1H), 1.15 (s, $3 \mathrm{H}), 0.93$ (t J=7 Hz, 3H); ${ }^{13} \mathbf{C} \mathbf{N M R}\left(100 \mathrm{MHz} \mathrm{CDCl}_{3}\right) \delta 165.2,162.6,152.7,144.0$ 
(143.9), 135.6 (135.7), 132.6, 130.4, 128.7, 128.6, 128.4, 127.9 (127.7), 122.9 (122.3), 121.8 (121.6), 119.2, 113.8, 86.8 (87.3), 82.5, 76.6 (75.4), 74.1, 71.1 (71.3), 62.5 (62.6), 55.4, 52.8, 29.3 (29.5), 14.1, 8.4; HRMS calculated for $\mathrm{C}_{31} \mathrm{H}_{35} \mathrm{NO}_{8}(\mathrm{M}+\mathrm{Na})$ : 550.2421, found $(\mathrm{FAB})$ : 550.2454 . The chemical shifts in parentheses are the shifts for the minor diastereomer.

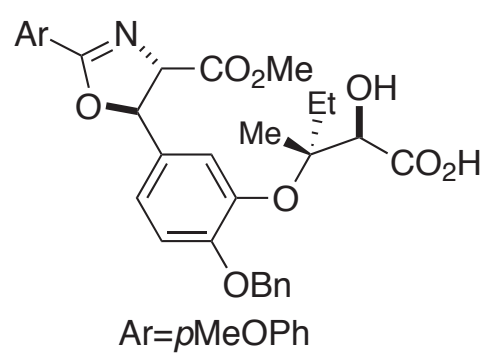

$(4 S, 5 R)-5-\{4-B e n z y l o x y-3-[(1 R, 2 R)-1-($ carboxyhydroxymethyl)-1-methylpropoxy]phenyl $\}-2-(4-$ methoxyphenyl)-4,5-dihydrooxazole-4-carboxylic acid methyl ester (61). Diol 42a (3.18 g, $5.79 \mathrm{mmol}, 1$ equiv) was dissolved in dichloromethane $(130 \mathrm{~mL})$ at 0 ${ }^{\circ} \mathrm{C}$. Saturated sodium bicarbonate solution $(130 \mathrm{~mL})$ was added followed by the addition of potassium bromide (138.1 mg, $1.16 \mathrm{mmol}, 0.2$ equiv), 2,2,6,6-tetramethyl-1piperidinyloxy, free radical (TEMPO) $(181.3 \mathrm{mg}, 1.16 \mathrm{mmol}, 0.2$ equiv). Sodium hypochlorite solution $(9.9 \mathrm{~mL}, 7.53 \mathrm{mmol}, 1.3$ equiv, commercial bleach, $5.25 \%$ by weight) was added with vigorous stirring. After 15 min, saturated sodium sulfite solution was added to quench excess oxidant. The layers were separated and the aqueous layer was extracted with chloroform $(3 \times 40 \mathrm{~mL})$. The combined organic layers were dried over anhydrous $\mathrm{Na}_{2} \mathrm{SO}_{4}$. The solvent was removed under reduced pressure and the crude product was directly used for the next oxidation reaction without further characterization. $\mathrm{R}_{\mathrm{f}}=0.8(1 / 2$ hexanes/ethyl acetate $)$.

The crude aldehyde was dissolved in tert-butyl alcohol $(393 \mathrm{~mL})$ and 2-methyl-2butene $(150 \mathrm{~mL})$ at room temperature. Sodium chlorite $(6.02 \mathrm{~g}, 53.3 \mathrm{mmol}, 9.2$ equiv, $\sim 80 \%$ tech grade) and sodium phosphate monobasic hydrate $(5.51 \mathrm{~g}, 40.0 \mathrm{mmol}, 6.9$ equiv) in water $(140 \mathrm{~mL})$ was added all at once. After stirring for $1 \mathrm{~h}, \mathrm{pH} 2.5$ phosphate buffer $(500 \mathrm{~mL})$ and ethyl acetate $(200 \mathrm{~mL})$ were added and the layers were separated. The aqueous layer was extracted with chloroform $(3 \times 100 \mathrm{~mL})$. The combined organic layers were dried over anhydrous $\mathrm{Na}_{2} \mathrm{SO}_{4}$. After removal of the solvent, the crude product was purified using silica gel column chromatography. TEMPO eluted first with 1/1 hexanes/ethyl acetate. Desired compound $61(3.11 \mathrm{~g}, 5.52 \mathrm{mmol}, 95 \%)$ started to elute using a gradient of $1 / 4$ hexanes/ethyl acetate to $100 \%$ ethyl acetate followed by $5 / 1$ to $2 / 1$ chloroform/methanol. $\mathrm{R}_{\mathrm{f}}=0.5$ (5/1 chloroform/methanol); IR (film) $2952 \mathrm{w}, 1742$ m, 1634 m, 1609 m, 1513 s, 1456 w, 1259 s, 1172 s, 1122 m, 1084 m, 1024 m, 842 w, $744 \mathrm{~m}, 698$ w; ${ }^{1} \mathbf{H}$ NMR $\left(500 \mathrm{MHz}, \mathrm{CDCl}_{3}\right) \delta 7.94(\mathrm{~d} \mathrm{~J}=7 \mathrm{~Hz}, 2 \mathrm{H}), 7.44-7.26(\mathrm{~m}, 5 \mathrm{H})$, $7.02(\mathrm{~d} \mathrm{~J}=16 \mathrm{~Hz}, 2 \mathrm{H}), 6.99-6.88(\mathrm{~m}, 3 \mathrm{H}), 5.72(\mathrm{br}, 1 \mathrm{H}), 5.08(\mathrm{br}, 2 \mathrm{H}), 4.71(\mathrm{br}, 1 \mathrm{H}), 4.24$ (d J=22 Hz, 1H), 3.81 (s, 3H), 3.79 (s, 3H), 2.00-1.65 (m, 2H), 1.20 (s, 3H), $0.97(\mathrm{~m}$, $3 \mathrm{H}) ;{ }^{13} \mathbf{C}$ NMR $\left(125 \mathrm{MHz}, \mathrm{CDCl}_{3}\right) \delta 171.7,165.6,162.9,152.6,143.7,135.9,132.8$, $130.8,129.0,127.9,121.9,119.3,114.0,86.7,82.8,76.7,71.4,55.7,53.1,29.3,20.0$, 19.5, 14.5, 8.4; HRMS calculated for $\mathrm{C}_{31} \mathrm{H}_{35} \mathrm{NO}_{9}(\mathrm{M}+\mathrm{H})$ : 564.2228 , found (FAB): 564.2252. 


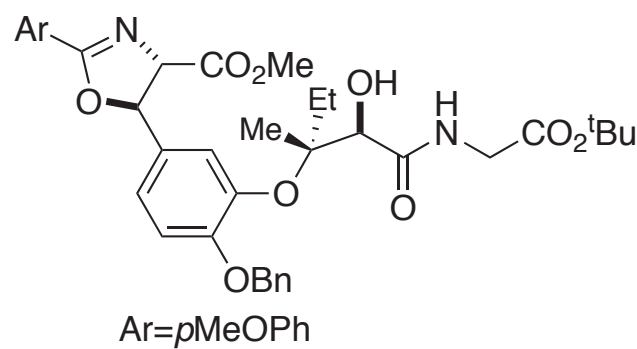

$(4 S, 5 R)-5-(4-B e n z y l o x y-3-\{(1 R)-1-[(R)-(t e r t-$ butoxycarbonylmethylcarbamoyl)hydroxyme thyl]-1-methylpropoxy\}phenyl)-2-(4methoxyphenyl)-4,5-dihydrooxazole-4carboxylic acid methyl ester (62). Free acid $61(3.11 \mathrm{~g}, 5.52 \mathrm{mmol}, 1$ equiv) and glycine tert-butyl ester hydrochloride $(1.11 \mathrm{~g}, 6.63$ mmol, 1.2 equiv) were dissolved in acetonitrile

$(55 \mathrm{~mL})$ at room temperature. Benzotriazole-1-yloxy-tris(dimethylamino)phosphonium hexafluorophosphate (BOP reagent) (3.66 g, $8.28 \mathrm{mmol}, 1.5$ equiv) was added, followed by the addition of diisopropylethylamine $(3.85 \mathrm{~mL}, 22.1 \mathrm{mmol}, 4$ equiv). After stirring for $1.5 \mathrm{~h}$, more glycine tert-butyl ester hydrochloride ( $462.7 \mathrm{mg}, 2.76 \mathrm{mmol}, 0.5$ equiv), BOP reagent $(1.22 \mathrm{~g}, 2.76 \mathrm{mmol}, 0.5$ equiv) and diisopropylethylamine $(962 \mu \mathrm{L}, 5.52$ mmol, 1 equiv) were added. The reaction was further stirred for another $5 \mathrm{~h}$ and then diluted with ethyl acetate $(75 \mathrm{~mL})$ before quenching with water $(75 \mathrm{~mL})$. The layers were separated and the aqueous layer was extracted with ethyl acetate $(2 \times 75 \mathrm{~mL})$. The organics were pooled washed with saturated sodium bicarbonate solution $(200 \mathrm{~mL})$ and with brine $(200 \mathrm{~mL})$, and dried over anhydrous $\mathrm{Na}_{2} \mathrm{SO}_{4}$. After the removal of the solvent under reduced pressure, the crude product was purified using silica gel column chromatography. Using a gradient of $2 / 1$ to $1 / 2$ hexanes/ethyl acetate, 62 (3.58 g, 5.29 mmol, 96\%) was obtained. $\mathrm{R}_{\mathrm{f}}=0.5$ (1/1 hexanes/ethyl acetate); IR (film) $3359 \mathrm{w}, 2976$ w, 1741 s, 1641 m, 1609 m, 1512 s, 1368 m, 1257 s, 1170 s, 1082 m, 1027 m, 844 m, 746 w; ${ }^{1} \mathbf{H}$ NMR $\left(500 \mathrm{MHz}, \mathrm{CDCl}_{3}\right) \delta 7.92(\mathrm{dd} \mathrm{J}=2,9 \mathrm{~Hz}, 2 \mathrm{H}), 7.71(7.65)(\mathrm{t} \mathrm{J}=6 \mathrm{~Hz}, 1 \mathrm{H})$, 7.36-7.29 (m, 5H), 7.03-6.90 (m, 3H), $6.87(\mathrm{~d} \mathrm{~J}=6 \mathrm{~Hz}, 2 \mathrm{H}), 5.70(\mathrm{t} \mathrm{J}=7 \mathrm{~Hz}, 1 \mathrm{H}), 5.02$ (dd $\mathrm{J}=2,10 \mathrm{~Hz}, 2 \mathrm{H}), 4.67(\mathrm{~d} \mathrm{~J}=7 \mathrm{~Hz}, 1 \mathrm{H}), 4.26(4.40)(\mathrm{d} \mathrm{J}=2 \mathrm{~Hz}, 1 \mathrm{H}), 4.13(4.25)(\mathrm{d} \mathrm{J}=4 \mathrm{~Hz}$, $1 \mathrm{H}), 3.80(\mathrm{~s}, 3 \mathrm{H}), 3.78(\mathrm{~s}, 3 \mathrm{H}), 3.70(\mathrm{dd} \mathrm{J}=5,8 \mathrm{~Hz}, 1 \mathrm{H}), 3.52(3.31)(\mathrm{dd} \mathrm{J}=6,18 \mathrm{~Hz}, 1 \mathrm{H})$, $1.95-1.88(1.83-1.77)(\mathrm{m}, 1 \mathrm{H}), 1.78-1.69(\mathrm{~m}, 1 \mathrm{H}), 1.32(1.38)(\mathrm{s}, 9 \mathrm{H}), 1.19(\mathrm{~s}, 3 \mathrm{H}), 0.98$ (t J=7 Hz, 3H); ${ }^{13} \mathbf{C}$ NMR $\left(125 \mathrm{MHz}, \mathrm{CDCl}_{3}\right) \delta 172.0$ (170.9), 168.2, 164.9, 162.4, 151.2 (152.3), 143.6 (143.5), 135.6 (135.4), 132.3 (132.2), 130.1, 128.6 (128.5), 128.3, 127.5 (127.7), 122.1, 120.3, 119.5, 118.8, 113.5, 113.4 (113.2), 86.6 (85.8), 82.3 (82.2), 81.4 (81.6), 76.4, 74.6 (72.7), 70.9 (70.8), 55.2, 52.6, 41.4, 40.1, 39.8 (39.9), 39.6 (39.4), 28.3 (28.6), 27.7 (27.8), 18.7 (19.0), 7.4 (7.8); HRMS calculated for $\mathrm{C}_{37} \mathrm{H}_{44} \mathrm{~N}_{2} \mathrm{O}_{10}(\mathrm{M}+\mathrm{Na})$ : 677.3074, found (FAB): 677.3080; The chemical shifts in parentheses are the shifts for the minor diastereomer.

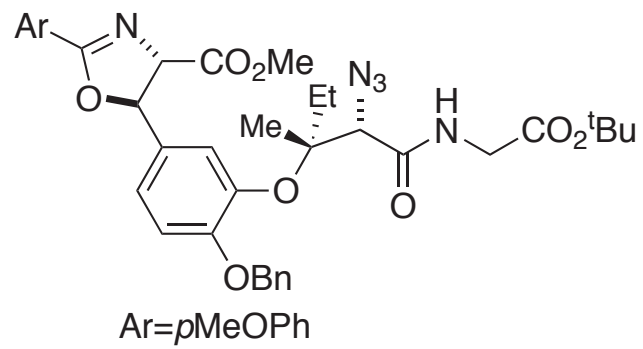

$(4 S, 5 R)-5-(3-\{(1 R)-1-[(S)-$ Azido- $($ tertbutoxycarbonymethylcarbamoyl)methyl]-1methylpropoxy\}-4-benzyloxyphenyl)-2-(4methoxyphenyl)-4,5-dihydrooxazole-4carboxylic acid methyl ester (63). Secondary alcohol 62 (3.58 g, $5.29 \mathrm{mmol}, 1$ equiv) was dissolved in dichloromethane $(133 \mathrm{~mL})$ at -20 ${ }^{\circ} \mathrm{C}$. Pyridine $(4.3 \mathrm{~mL}, 52.9 \mathrm{mmol}, 10$ equiv)

was added followed by the addition of trifluoromethanesulfonic anhydride $(2.67 \mathrm{ml}, 15.9$ mmol, 3 equiv). Temperature was carefully maintained at $-20{ }^{\circ} \mathrm{C}$ over $22 \mathrm{~h}$. The 
reaction was quenched with saturated sodium bicarbonate solution $(75 \mathrm{~mL})$ and warmed to room temperature. The layers were separated and the aqueous layer was extracted with chloroform $(3 \times 75 \mathrm{~mL})$. The combined organic layers were dried over anhydrous $\mathrm{Na}_{2} \mathrm{SO}_{4}$. After the removal of solvent under reduced pressure, the crude triflate was purified using silica gel column chromatography. A byproduct and pyridine eluted using $3 / 1$ hexanes/ethyl acetate, followed by the desired triflate $(3.32 \mathrm{~g}, 4.10 \mathrm{mmol}, 78 \%)$, which eluted with a gradient of $3 / 1$ to $1 / 1$ hexanes/ethyl acetate. Recovered starting material 62 (422.5 mg, $0.624 \mathrm{mmol}, 12 \%$ ) eluted using $1 / 2$ hexanes/ethyl acetate. The triflate was used without further characterization. $R_{\mathrm{f}}=0.6$ ( $1 / 1$ hexanes/ethyl acetate).

The triflate (3.32 g, $4.10 \mathrm{mmol}, 1$ equiv) was dissolved in 1,3-dimethyl-3,4,5,6tetrahydro-2 $(H)$-pyrimidinone (DMPU) $(20 \mathrm{~mL})$. Solid lithium azide (prepared by lyophilizing an aqueous solution-CAUTION: Potential for explosion) (1.61 g, 32.8 mmol, 8 equiv) was added and the reaction was stirred at room temperature for 2.5 days. The reaction was loaded directly onto a silica gel column and eluted with $1 / 2$ hexanes/ethyl acetate to remove most of the reaction solvent, DMPU. The product fractions were concentrated and the crude product was purified using silica gel column chromatography. Trace amount of the recovered triflate eluted using 1/4/2 hexanes/benzene/ether. The product 63 (2.06 g, $2.94 \mathrm{mmol}, 72 \%)$ eluted using 1/8/4 hexanes/benzene/ether. $R_{\mathrm{f}}=0.6$ (2/1 benzene/ether, developed twice); IR (film) $3364 \mathrm{w}$, 2978 w, 2116 s, 1739 m, 1665 m, 1609 m, 1506 m, 1513 m, 1368 m, 1257 s, 1224 s, 1170 s, 1082 m, 1027 m, 843 w, 749 w, 699 w; ${ }^{1} \mathbf{H}$ NMR (400 MHz, CDCl $) \delta 7.96$ (dt $\mathrm{J}=2,9 \mathrm{~Hz}, 2 \mathrm{H}), 7.41-7.28(\mathrm{~m}, 6 \mathrm{H}), 7.06-6.91(\mathrm{~m}, 3 \mathrm{H}), 6.91(\mathrm{dt} \mathrm{J}=3,9 \mathrm{~Hz}, 2 \mathrm{H}), 5.75$ (d $\mathrm{J}=7 \mathrm{~Hz}, 1 \mathrm{H}), 5.05(\mathrm{~d} \mathrm{~J}=10 \mathrm{~Hz}, 2 \mathrm{H}), 4.72(\mathrm{dd} \mathrm{J}=3,7 \mathrm{~Hz}, 1 \mathrm{H}), 4.33(4.28)(\mathrm{s}, 1 \mathrm{H}), 3.83(\mathrm{~s}$, $3 \mathrm{H}), 3.82(\mathrm{~s}, 3 \mathrm{H}), 3.71(\mathrm{dd} \mathrm{J}=5,7 \mathrm{~Hz}, 1 \mathrm{H}), 3.57(\mathrm{dd} \mathrm{J}=5,11 \mathrm{~Hz}, 1 \mathrm{H}), 1.89-1.79(\mathrm{~m}, 1 \mathrm{H})$, 1.81-1.73 (m, 1H), $1.41(1.39)(\mathrm{s}, 9 \mathrm{H}), 1.2(1.3)(\mathrm{s}, 3 \mathrm{H}), 0.96(0.95)(\mathrm{t}, \mathrm{J}=7 \mathrm{~Hz}, 3 \mathrm{H}) ;{ }^{13} \mathrm{C}$ NMR $\left(100 \mathrm{MHz} \mathrm{CDCl}_{3}\right) \delta 171.5,168.3$ (168.4), 167.4 (167.8), 165.2, 162.6, 152.9 (152.4), 143.4 (143.5), 136.0, 132.3 (132.2), 130.4, 128.7 (128.8), 128.4, 127.8, 122.9, 121.9 (121.8), 121.5, 119.1, 113.8 (113.5), 85.9 (85.7), 82.5, 82.0 (81.9), 70.9, 69.1 (68.8), 55.4, 52.8, 41.8 (41.6), 29.2, 28.0, 20.3 (20.1), 8.3 (8.0); HRMS calculated for $\mathrm{C}_{37} \mathrm{H}_{43} \mathrm{~N}_{5} \mathrm{O}_{9}(\mathrm{M}+\mathrm{H}): 702.3133$, found $(\mathrm{FAB}): 702.3165$. The chemical shifts in parentheses are the shifts for the minor diastereomer.

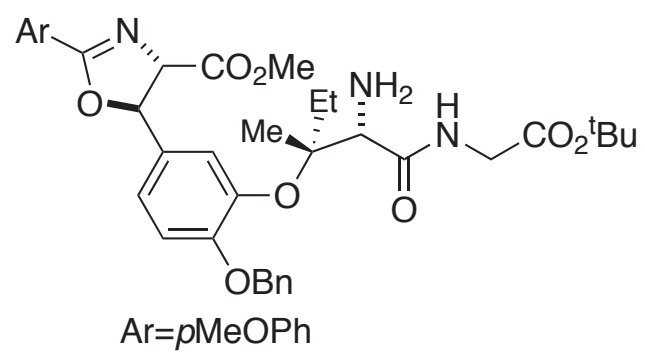

$(4 S, 5 R)-5-(3-\{(1 R)-1-[(S)-((2 S)-2-A z i d o-3-$ methylbutyrylamino) -(tert-butoxycarbonylmethylcarbamoyl)methyl]-1-methylpropoxy -4-benzyloxyphenyl)-2-(4-methoxyphenyl)-4,5-dihydrooxazole-4-carboxylic acid methyl ester (75). Azide 63 (2.06 g, 2.94 mmol, 1 equiv) was dissolved in THF $(21 \mathrm{~mL})$ and water $(8 \mathrm{~mL})$ at room temperature.

Trimethylphosphine (1 M solution in THF) $(8.8 \mathrm{~mL}, 8.8 \mathrm{mmol}, 4$ equiv) was added and the reaction was allowed to stir $24 \mathrm{~h}$. THF was removed under reduced pressure. Ethyl acetate $(80 \mathrm{~mL})$ and saturated sodium bicarbonate solution $(80 \mathrm{~mL})$ were added. The layers were separated and the aqueous layer was extracted with ethyl acetate $(2 \times 80 \mathrm{~mL})$. The combined organic layers were washed with brine $(80 \mathrm{~mL})$ and dried over anhydrous $\mathrm{Na}_{2} \mathrm{SO}_{4}$. Solvents were removed under reduced pressure and the crude product was 
loaded onto a silica gel column. Trace impurities were eluted using $1 / 2$ hexanes/ethyl acetate followed by the desired amine $(1.54 \mathrm{~g}, 2.28 \mathrm{mmol}, 78 \%)$. Polarity was increased to $1 / 4$ then $5 / 1$ chloroform/methanol to complete elution of 75 . $\mathrm{R}_{\mathrm{f}}=0.2(1 / 2$ hexanes/ethyl acetate); IR (film) 3368 w, 2978 w, 1739 m, 1641 w, 1609 w, 1512 s, 1368 w, 1256 s, 1169 s, 1082 m, 1026 w, 843 w, 749 w; ${ }^{1} \mathbf{H}$ NMR $\left(500 \mathrm{MHz}, \mathrm{CDCl}_{3}\right) \delta 7.96(\mathrm{dd} \mathrm{J}=1,9$ $\mathrm{Hz}, 2 \mathrm{H}), 7.83$ (7.72) (t J=6 Hz, 1H), 7.40-7.24 (m, 5H), 7.04-6.90 (m, 3H), 6.91 (dd J=1, $8 \mathrm{~Hz}, 2 \mathrm{H}), 5.73(\mathrm{~d} \mathrm{~J}=8 \mathrm{~Hz}, 1 \mathrm{H}), 5.01(5.05)(\mathrm{s}, 2 \mathrm{H}), 4.72(\mathrm{dd} \mathrm{J}=2,7 \mathrm{~Hz}, 1 \mathrm{H}), 3.83(\mathrm{~s}, 3 \mathrm{H})$, $3.81(\mathrm{~s}, 3 \mathrm{H}), 3.80-3.75(\mathrm{~m}, 1 \mathrm{H}), 3.68-3.59(\mathrm{~m}, 1 \mathrm{H}), 1.92-1.87(\mathrm{~m}, 1 \mathrm{H}), 1.83(\mathrm{br}, 2 \mathrm{H})$, 1.83-1.73 (m, 1H), $1.41(1.38)(\mathrm{s}, 9 \mathrm{H}), 1.25(1.17)(\mathrm{s}, 3 \mathrm{H}), 1.01(0.93)(\mathrm{t} \mathrm{J}=7 \mathrm{~Hz}, 3 \mathrm{H}) ;{ }^{13} \mathbf{C}$ NMR $\left(125 \mathrm{MHz} \mathrm{CDCl}_{3}\right) \delta 173.0$ (173.2), 171.5, 168.9, 165.2, 162.6, 153.1, 152.4, 144.0 (144.1), 136.1, 132.3 (132.2), 130.4, 128.6 (128.7), 128.4 (128.3), 127.8, 122.2 (121.4), 121.0 (121.2), 119.2, 113.8, 113.5 (113.4), 86.4, 82.5 (82.6), 81.6 (81.8), 76.6, 70.9, 60.2 (60.6), 55.4, 52.8, 41.7, 29.6 (28.6), 28.0, 19.6 (19.9), 8.2 (8.1); HRMS calculated for $\mathrm{C}_{37} \mathrm{H}_{45} \mathrm{~N}_{3} \mathrm{O}_{9}(\mathrm{M}+\mathrm{H}):$ 676.3228, found $(\mathrm{FAB}):$ 676.3236. The chemical shifts in parentheses are the shifts for the minor diastereomer.

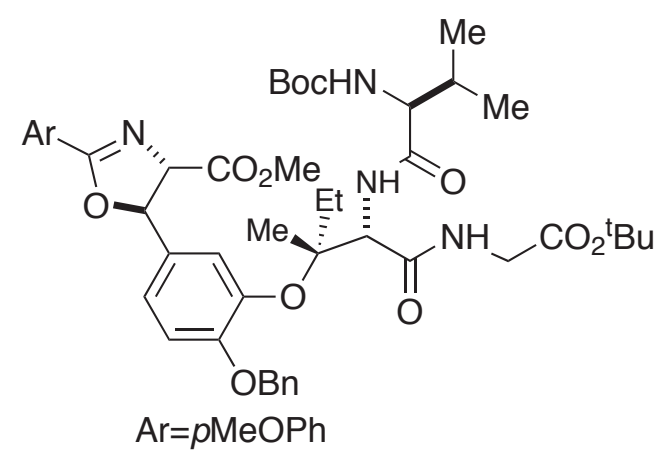

$(4 S, 5 R)-5-(3-\{(1 R)-1-[(S)-((2 S)-A z i d o-3-$ methylbutyrylamino)-(tert-butoxycarbonylmethylcarbamoyl)methyl]-1methylpropoxy\}-4-benzyloxyphenyl)-2-(4methoxyphenyl)-4,5-dihydrooxazole-4carboxylic acid methyl ester (64). Amine 75 (679 mg, $1.00 \mathrm{mmol}, 1$ equiv) and Boc-valine (326 mg, $1.50 \mathrm{mmol}, 1.5$ equiv) were dissolved in acetonitrile $(10 \mathrm{~mL})$ at room temperature. BOP reagent $(889 \mathrm{mg}, 2.01 \mathrm{mmol}, 4$ equiv) and diisopropylethylamine (700 $\mu \mathrm{L}, 4.02 \mathrm{mmol}, 2$ equiv) were added and the reaction was stirred $1 \mathrm{~h}$. The reaction was diluted with ethyl acetate $(50 \mathrm{~mL})$ and saturated sodium bicarbonate solution $(50 \mathrm{~mL})$ was added. The layers were separated and the aqueous layer extracted with ethyl acetate $(2 \times 50 \mathrm{~mL})$. The combined organic layers were washed once with brine $(30 \mathrm{~mL})$ and dried over anhydrous $\mathrm{Na}_{2} \mathrm{SO}_{4}$. Solvents were removed under reduced pressure and the crude product was loaded onto a silica gel column. Trace impurities were removed eluting with 2:1 hexanes/ethyl acetate. The desired product 64 (911.9 $\mathrm{mg}, 1.04 \mathrm{mmol}$, quant.) was obtained upon elution with $1 / 1$ to $1 / 2$ hexanes/ethyl acetate. $R_{\mathrm{f}}=0.6$ (1/5 hexanes/ethyl acetate); $\mathbf{I R}$ (film) $3371 \mathrm{~m}, 2975 \mathrm{~m}$, $1743 \mathrm{~s}, 1664 \mathrm{~s}, 1610 \mathrm{~m}, 1512 \mathrm{~s}, 1456 \mathrm{w}, 1367 \mathrm{~m}, 1257 \mathrm{~s}, 1170 \mathrm{~s}, 1083 \mathrm{w}, 1026 \mathrm{~s}, 1009$ m, 845 w, 750 w, 700 w; ${ }^{1} \mathbf{H}$ NMR $\left(500 \mathrm{MHz}, \mathrm{CDCl}_{3}\right) \delta 7.98$ (dd J=2, $\left.9 \mathrm{~Hz}, 2 \mathrm{H}\right), 7.49-$ 7.33 (m, 6H), 7.09-6.97 (m, 3H), 6.95 (dd J=1, $9 \mathrm{~Hz}, 2 \mathrm{H}), 5.75$ (dd J=3, $7 \mathrm{~Hz}, 1 \mathrm{H}), 5.58$ (5.53) (d J=8 Hz, 1H), 5.18 (5.24) (d J=9 Hz, 2H), 4.77 (4.96) (d J=9 Hz, 1H), 4.73 (d $\mathrm{J}=7 \mathrm{~Hz}, 1 \mathrm{H}), 3.88(\mathrm{~s}, 3 \mathrm{H}), 3.85(\mathrm{~s}, 3 \mathrm{H}), 3.75-3.70(\mathrm{~m}, 1 \mathrm{H}), 3.65-3.57(\mathrm{~m}, 1 \mathrm{H}), 3.48-3.41$ $(\mathrm{m}, 1 \mathrm{H}), 1.83-1.77(2.01-1.92)(\mathrm{m}, 1 \mathrm{H}), 1.71-1.66(\mathrm{~m}, 1 \mathrm{H}), 1.41(1.38)(\mathrm{s}, 9 \mathrm{H}), 1.29$ (1.22) (s, 3H), 0.98 (t J=7 Hz, 3H), 0.92 (d J=7 Hz, 3H), 0.88 (d J=7 Hz, 3H); ${ }^{13} \mathbf{C ~ N M R}$ $\left(125.5 \mathrm{MHz} \mathrm{CDCl}_{3}\right) \delta 171.8,170.6,169.1,167.9$ (168.1), 164.7, 162.1, 155.5, 135.7, 129.9, 128.3 (128.2), 127.7 (127.9), 127.1, 122.9, 118.6, 113.3, 113.0, 85.7 (86.2), 82.0, 81.1, 79.1, 76.1, 70.4, 58.2, 54.9, 52.3, 41.5 (41.3), 39.8 (40.0), 39.5 (39.6), 39.3 (39.1), $30.2,27.8$ (27.4), 19.7 (19.8), 18.8, 17.4, 7.9; HRMS calculated for $\mathrm{C}_{47} \mathrm{H}_{62} \mathrm{~N}_{4} \mathrm{O}_{12}$ 
$(\mathrm{M}+\mathrm{Na}): 897.4262$, found $(\mathrm{FAB}): 897.4275$. The chemical shifts in parentheses are the shifts for the minor diastereomer.

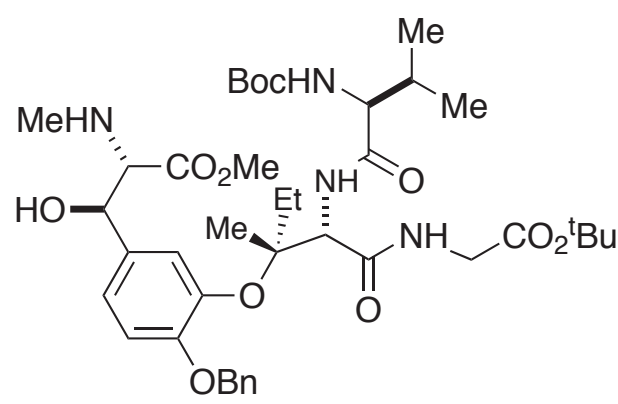

$(2 S, 3 R)-3-(3-\{(1 R)-1-[(S)-((2 S)-2-A z i d o-3-$ methylbutyrylamino)-(tert-butoxycarbonylmethylcarbamoyl)methyl]-1methylpropoxy\}-4-benzyloxyphenyl)-3hydroxy-2-methylaminopropionic acid methyl ester (65). Oxazoline 64 (911.9 mg, $1.04 \mathrm{mmol}$, 1 equiv) was dissolved in dichloromethane $(20$ $\mathrm{mL}$ ) at room temperature. Methyl trifluoromethanesulfonate $(141.5 \mu \mathrm{L}, 1.25 \mathrm{mmol}$,

1.2 equiv, freshly distilled from calcium hydride) was added and the reaction was stirred for $2 \mathrm{~h}$. Toluene was added and the reaction mixture was azeotroped and dried under high vacuum $(0.5 \mathrm{mmHg})$. The residue was dissolved in dichloromethane $(30 \mathrm{~mL})$ at 0 ${ }^{\circ} \mathrm{C}$. Saturated sodium bicarbonate solution $(30 \mathrm{~mL})$ and sodium borohydride $(78.7 \mathrm{mg}$, $2.08 \mathrm{mmol}, 2$ equiv) were added at once with vigorous stirring. Bubbling ensued and after $15 \mathrm{~min}$, the layers were separated. The aqueous layer was extracted with dichloromethane $(2 \times 30 \mathrm{~mL})$. The combined organic layers were dried over anhydrous $\mathrm{Na}_{2} \mathrm{SO}_{4}$ and the solvent removed under reduced pressure to leave a white foam. The crude product was purified using silica gel column chromatography. The desired $(4 S, 5 R)-5-(3-\{(1 R)-1-[(S)-((2 S)-2$-azido-3-methylbutyrylamino)-(tert-

butoxycarbonylmethylcarbamoyl)methyl]-1-methylpropoxy\}-4-benzyloxyphenyl)-2-(4methoxyphenyl)-3-methyloxazolidine-4-carboxylic acid methyl ester (759 mg, 0.852 mmol, $82 \%)$ was obtained upon elution with $2 / 1$ to $1 / 1$ hexanes/ethyl acetate. $R_{\mathrm{f}}=0.8(1 / 2$ hexanes/ethyl acetate).

$(4 S, 5 R)-5-(3-\{(1 S)-1-[(S)-((2 S)-2-$ Azido-3-methylbutyrylamino)-(tert-butoxycarbonylmethylcarbamoyl)methyl]-1-methylpropoxy\}-4-benzyloxyphenyl)-2-(4methoxyphenyl)-3-methyloxazolidine-4-carboxylic acid methyl ester (759 mg, 0.852 $\mathrm{mmol})$ was dissolved in THF (28 mL) at room temperature. Oxalic acid (22 $\mathrm{mL}, 2.17$ mmol, 2.5 equiv, $0.1 \mathrm{~N}$ solution in water) was added and the reaction was stirred $12 \mathrm{~h}$. THF was removed under reduced pressure, and dichloromethane $(50 \mathrm{~mL})$ and saturated sodium bicarbonate solution $(15 \mathrm{~mL})$ were added. The layers were separated and the aqueous layer was extracted with dichloromethane $(3 \times 30 \mathrm{~mL})$. The combined organic layers were dried over anhydrous $\mathrm{Na}_{2} \mathrm{SO}_{4}$. The solvent was removed under reduced pressure and the crude products were purified using silica gel column chromatography. Using 1/1 hexanes/ethyl acetate, $p$-methoxybenzaldehyde and the over-reduced product that had formed in the methylation step eluted. The desired product 65 (591 mg, 0.764 mmol, $90 \%, 74 \%$ over 2 steps) was obtained upon elution with a gradient of $1 / 4$ hexanes/ethyl acetate, $100 \%$ ethyl acetate and 20/1 chloroform/methanol. $R_{\mathrm{f}}=0.2(1 / 2$ hexanes/ethyl acetate); IR (film) 3347 br, $2971 \mathrm{w}, 2358 \mathrm{~m}, 1672 \mathrm{~m}, 1510 \mathrm{~s}, 1368 \mathrm{~m}, 1259$ m, $1161 \mathrm{~s}, 1015 \mathrm{~m}, 750 \mathrm{w} ;{ }^{1} \mathbf{H}$ NMR $\left(500 \mathrm{MHz}, \mathrm{CDCl}_{3}\right) \delta$ 7.43-7.29 (m, 5H), 7.14-6.86 $(\mathrm{m}, 3 \mathrm{H}), 5.17(\mathrm{~d}, \mathrm{~J}=11 \mathrm{~Hz}, 1 \mathrm{H}), 5.07(5.11)(\mathrm{s}, 2 \mathrm{H}), 5.03(\mathrm{t}, \mathrm{J}=7 \mathrm{~Hz}, 1 \mathrm{H}), 4.81$ (4.92) (d, $\mathrm{J}=8 \mathrm{~Hz}, 1 \mathrm{H}), 3.88-3.83(4.35-4.32)(\mathrm{m}, 1 \mathrm{H}), 3.73$ (3.75) (s, 3H), $3.53(\mathrm{~d}, \mathrm{~J}=2 \mathrm{~Hz}, 1 \mathrm{H})$, 3.45-3.30 (m, 1H), $3.23(3.13)(\mathrm{d}, \mathrm{J}=5 \mathrm{~Hz}, 1 \mathrm{H}), 2.36(\mathrm{~s}, 3 \mathrm{H}), 1.76-1.64$ (1.90-1.78) (m, 2H), 1.38 (1.35) (s, 9H), 1.25 (1.22) (s, 3H), $0.96(\mathrm{t}, \mathrm{J}=7 \mathrm{~Hz}, 3 \mathrm{H}), 0.90(\mathrm{~d}, \mathrm{~J}=7 \mathrm{~Hz}, 3 \mathrm{H})$, 
$0.86(\mathrm{~d}, \mathrm{~J}=7 \mathrm{~Hz}, 3 \mathrm{H}) ;{ }^{13} \mathbf{C}$ NMR $\left(125.5 \mathrm{MHz}, \mathrm{CDCl}_{3}\right) \delta 171.8,170.3,169.4,168.5$ (168.9), 155.8, 143.4, 128.6 (128.8), 128.5 (127.7), 124.1, 122.3, 121.2, 112.9, 97.5, 86.4, 81.5, 80.2 (80.3), 79.7, 75.1, 71.0, 70.4, 62.9, 60.1, 58.8, 52.5, 52.1, 51.8, 42.1 (41.9), 38.1, 35.1, 31.1, 30.5, 28.6, 28.2 (28.0), 26.6, 19.3 (20.0), 17.8; HRMS calculated for $\mathrm{C}_{40} \mathrm{H}_{60} \mathrm{~N}_{4} \mathrm{O}_{11}(\mathrm{M}+\mathrm{H})$ : 773.4337 , found $(\mathrm{FAB}): 773.4345$. The chemical shifts in parentheses are the shifts for the minor diastereomer.

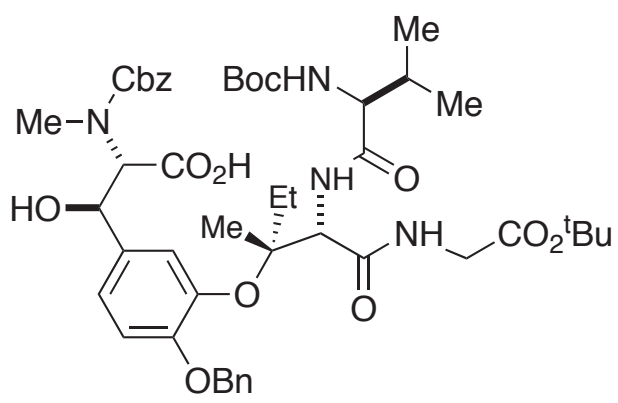

$(2 S, 3 R)-3-(3-\{(1 R)-1-[(S)-((2 S)-2-A z i d o-3-$ methylbutyrylamino)-(tert-butoxycarbonylmethylcarbamoyl)methyl]-1methylpropoxy\}-4-benzyloxyphenyl)-2(benzyloxycarbonylmethylamino)-3-

hydroxypropionic acid (76). Methyl ester 65 (591 $\mathrm{mg}, 0.764 \mathrm{mmol}, 1$ equiv) was dissolved in THF $(40 \mathrm{~mL})$ at $0{ }^{\circ} \mathrm{C}$. Lithium hydroxide $(0.1 \mathrm{~N}$ solution in water) $(19.1 \mathrm{~mL}, 1.91 \mathrm{mmol}, 2.5$ equiv) was added and the reaction was stirred for $3 \mathrm{~h}$ at $0{ }^{\circ} \mathrm{C}$. At this point, TLC showed complete methyl ester hydrolysis, so benzyl chloroformate ( $327 \mu \mathrm{L}, 2.29 \mathrm{mmol}, 3$ equiv) was added and the reaction was stirred for $3 \mathrm{~h}$. At this time, an additional aliquots of benzyl chloroformate were added ( $109 \mu \mathrm{L}, 0.764 \mathrm{mmol}, 1$ equiv) every $1 \mathrm{~h}$ over $2 \mathrm{~h}$. After stirring one additional hour, the reaction was diluted with dichloromethane $(50 \mathrm{~mL})$ and pH 2.5 phosphate buffer was added $(100 \mathrm{~mL})$. The layers were separated and the aqueous layer was extracted with dichloromethane $(3 \times 50 \mathrm{~mL})$. The combined organic layers were dried over anhydrous $\mathrm{Na}_{2} \mathrm{SO}_{4}$. The solvent was removed under reduced pressure and the crude product was purified using silica gel column chromatography. Excess benzyl chloroformate was eluted using $1 / 2$ hexanes/ethyl acetate, and the desired product $76(420 \mathrm{mg}, 0.470 \mathrm{mmol}, 62 \%)$ was obtained upon elution with a gradient of 25/1 to 10/1 chloroform/methanol. $\mathrm{R}_{\mathrm{f}}=0.6$ (5/1 chloroform/methanol); IR (film) $3332 \mathrm{br}$, 2974 m, 2937 m, 2256 w, 1668 s, 1505 s, 1455 w, 1391 w, 1367 m, 1302 w, 1257 m, $1220 \mathrm{~m}, 1158 \mathrm{~s}, 1026 \mathrm{~m}, 1007 \mathrm{~m}, 850 \mathrm{w}, 823 \mathrm{w}, 770 \mathrm{~m}, 699 \mathrm{w} ;{ }^{1} \mathbf{H}$ NMR $(500 \mathrm{MHz}$, DMSO- $\left.\mathrm{d}_{6}, 80{ }^{\circ} \mathrm{C}\right) \delta$ 7.98-7.55 (m, $\left.1 \mathrm{H}\right), 7.46(\mathrm{dd}, \mathrm{J}=7,17 \mathrm{~Hz}, 1 \mathrm{H}), 7.39-6.90(\mathrm{~m}, 6 \mathrm{H})$, $5.16(5.02)(\mathrm{s}, 2 \mathrm{H}), 4.89-4.64(\mathrm{~m}, 3 \mathrm{H}), 3.85(\mathrm{~d}, \mathrm{~J}=27 \mathrm{~Hz}, 1 \mathrm{H}), 3.74-3.50(\mathrm{~m}, 2 \mathrm{H}), 2.90(\mathrm{~s}$, $3 \mathrm{H}), 1.90-1.64(\mathrm{~m}, 2 \mathrm{H}), 1.38(\mathrm{~s}, 18 \mathrm{H}), 1.21(\mathrm{~s}, 3 \mathrm{H}), 0.89-0.83(\mathrm{~m}, 6 \mathrm{H}) ;{ }^{13} \mathbf{C}$ NMR $(125.5$ $\left.\mathrm{Hz}, \mathrm{DMSO}-\mathrm{d}_{6}, 80{ }^{\circ} \mathrm{C}\right) \delta 171.6,169.0,168.7,167.9,155.0,150.6,142.6,136.6,135.1$, $127.8,127.1,126.5,123.0,121.6,113.2,84.6,80.2,77.8,70.1,65.6,63.9,57.8,29.7$, 28.4, 27.8, 27.6, 27.4, 27.2, 18.7, 17.6, 7.9; HRMS calculated for $\mathrm{C}_{47} \mathrm{H}_{64} \mathrm{~N}_{4} \mathrm{O}_{13}(\mathrm{M}+\mathrm{Na})$ : 915.4368, found (FAB): 915.4408. The chemical shifts in parentheses are the shifts for the minor diastereomer. 


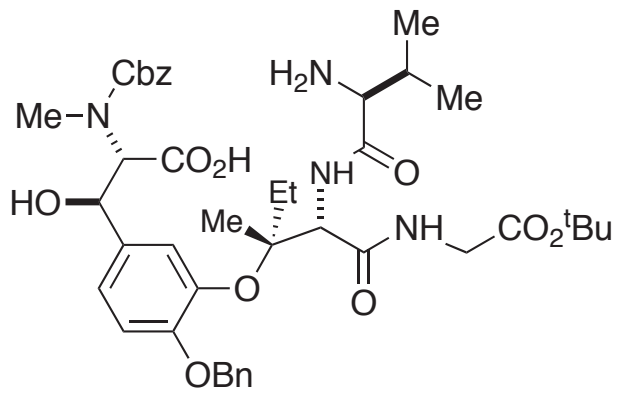

$(2 S, 3 R)-3-(3-\{(1 R)-1-[(S)-((2 S)-2-A z i d o-3-$ methylbutyrylamino)-(tert-butoxycarbonylmethylcarbamoyl)methyl]-1-methylpropoxy\}-4-benzyloxyphenyl)-2-(benzyloxycarbonylmethylamino)-3-hydroxypropionic acid (66). Carbamate 76 (200 $\mathrm{mg}$, $0.224 \mathrm{mmol}, 1$ equiv) was dissolved in dichloromethane $(2.6 \mathrm{~mL})$ and cooled to $0^{\circ} \mathrm{C}$. Pre-chilled trifluoroacetic acid was added $(640$ diluted with pre-chilled dichloromethane $(50 \mathrm{~mL})$ and poured into saturated sodium bicarbonate solution $(50 \mathrm{~mL})$ at $0^{\circ} \mathrm{C}$. The emulsion was filtered through a pad of Celite and the layers separated. The aqueous layer was extracted with ethyl acetate $(2 \times 50$ $\mathrm{mL})$. The combined organics were washed once with brine $(20 \mathrm{~mL})$ before drying them over anhydrous $\mathrm{Na}_{2} \mathrm{SO}_{4}$. Solvents were removed under reduced pressure, and the crude loaded onto a silica gel column. Trace impurities eluted using 50/1 chloroform/methanol and then a gradient of $25 / 1$ to $2 / 1$ chloroform/methanol eluted $\mathbf{6 6}(148 \mathrm{mg}, 0.186 \mathrm{mmol}$, $83 \%$ ) was obtained. The two diastereomers showed different $R_{\mathrm{f}}$ values on TLC but could not be separated. $\mathrm{R}=0.5$ (major isomer) (5/1 chloroform $/$ methanol), $\mathrm{R}_{\mathrm{f}}=0.4$ (minor isomer) (5/1 chloroform/methanol); IR (film) 3364 br, s, 3064 w, 2972 m, 2256 w, 2128 w, $1742 \mathrm{~m}, 1673 \mathrm{~s}, 1505 \mathrm{~m}, 1470 \mathrm{w}, 1455 \mathrm{w}, 1392 \mathrm{~m}, 1368 \mathrm{~m}, 1307 \mathrm{w}, 1258 \mathrm{~m}, 1221 \mathrm{~m}$, 1154 s, 1050 m, 1026 s, 1004 s, 847 w, 825 w, 765 m, 698 w, 666 w, 623 w; ${ }^{1}$ H NMR $\left(500 \mathrm{MHz}\right.$, DMSO-d 6 , $\left.80{ }^{\circ} \mathrm{C}\right) \delta 7.83(7.90)(\mathrm{br}, 1 \mathrm{H}), 7.45(\mathrm{dd}, \mathrm{J}=5,7 \mathrm{~Hz}, 1 \mathrm{H}), 7.37-7.16$ (m, 4H), 6.93 (7.11) (br, 2H), $5.07(5.13)(\mathrm{s}, 2 \mathrm{H}), 4.94(\mathrm{~s}, 1 \mathrm{H}), 4.68$ (br, 1H), $3.60(3.69)$ (dd, J=6, $17.5 \mathrm{~Hz}, 2 \mathrm{H}), 3.13$ (3.31) (d, J=4.5 Hz, 1H), 2.86 (2.75) (s, 3H), 2.07-1.98 (m, $1 \mathrm{H}), 1,83-1.74(1.67-1.60)(\mathrm{m}, 2 \mathrm{H}), 1.38$ (1.36) (s, 9H), 1.27 (1.20) (s, 3H), 0.88 (0.92) $(\mathrm{d}, \mathrm{J}=7 \mathrm{~Hz}, 3 \mathrm{H}), 0.82(0.90)(\mathrm{t}, \mathrm{J}=6 \mathrm{~Hz}, 3 \mathrm{H}), 0.78(\mathrm{~d}, \mathrm{~J}=7 \mathrm{~Hz}, 3 \mathrm{H}) ;{ }^{13} \mathbf{C}$ NMR $(125.5$ MHz, DMSO-d $\left.{ }_{6}, 80{ }^{\circ} \mathrm{C}\right) \delta 173.1,172.5,190.0,168.1,150.6,142.7$ (142.8), 136.8, 135.3, 127.8, 127.2, 126.6, 122.8, 121.7, 113.3, 84.2, 80.2 (80.1), 72.1 (72.3), 70.1 (70.0), 65.5, 63.9, 57.7, 30.5 (30.7), 28.6, 27.6, 27.4 (27.2), 27.0, 18.7, 16.3, 8.0; HRMS calculated for $\mathrm{C}_{42} \mathrm{H}_{56} \mathrm{~N}_{4} \mathrm{O}_{11}(\mathrm{M}+\mathrm{Na})$ : 815.3838, found (FAB): 815.3798. The chemical shifts in parentheses are the shifts for the minor diastereomer.

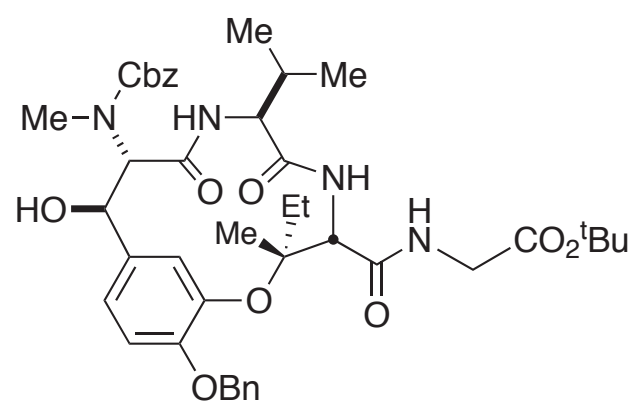

$\{[15-$ Benzyloxy-10-(benzyloxycarbonylmethylamino)-3-ethyl-11-hydroxyl-7isopropyl-3-methyl-6,9-dioxo-2-oxa-5,8-diazabicyclo[10.3.1] hexadeca-[(16),12,14-triene-4carbonyl]amino\}acetic acid tert-butyl ester (67). 7-Azabenzotriazol-1-yloxy-tris(pyrrolidino)phosphonium hexafluorophosphate (PyAOP) (253.4 mg, $0.486 \mathrm{mmol}, 3$ equiv), DMF (56 mL) and diisopropylethylamine $(141 \mu \mathrm{L}, 0.808$ mmol, 5 equiv) were combined. Amino acid 66 (128.1 mg, $0.162 \mathrm{mmol}, 1$ equiv) in DMF $(6 \mathrm{~mL})$ was added slowly using a syringe pump $(0.4 \mathrm{~mL} / \mathrm{h})$. When the addition was complete, the syringe was rinsed with DMF ( $5 \mathrm{~mL}$ ) and added over $1 \mathrm{~h}$. The mixture was 
stirred for one additional hour and then was diluted with diethyl ether $(60 \mathrm{~mL})$ and quenched with water $(60 \mathrm{~mL})$. The layers were separated and the aqueous layer was extracted with diethyl ether $(3 \times 60 \mathrm{~mL})$. The combined organic layers were washed once with brine $(30 \mathrm{~mL})$ and dried over anhydrous $\mathrm{Na}_{2} \mathrm{SO}_{4}$. After removal of solvents under reduced pressure, the crude material was purified using silica gel column chromatography. Using a gradient of $3 / 2$ to $1 / 2$ hexanes/ethyl acetate, 67 (66 mg, 0.0852 mmol, 56\%) was obtained. Some starting amino acid $66(7.3 \mathrm{mg}, 0.00921 \mathrm{mmol}, 6 \%)$ was recovered from the syringe used for the slow addition. $\mathrm{R}_{\mathrm{f}}=0.4(1 / 2$ hexanes/ethyl acetate); IR (film) 3319 br, $2971 \mathrm{~m}, 1660 \mathrm{~s}, 1504 \mathrm{~m}, 1261 \mathrm{~m}, 1222 \mathrm{~m}, 1154 \mathrm{~s}, 1026 \mathrm{~m}$, $738 \mathrm{w} ;{ }^{1} \mathbf{H}$ NMR $\left(500 \mathrm{MHz}, \mathrm{DMSO}_{-}, 80^{\circ} \mathrm{C}\right) \delta 8.01(7.93)(\mathrm{t}, \mathrm{J}=5.5 \mathrm{~Hz}, 1 \mathrm{H}), 7.76$ (7.86) (d, J=8 Hz, 1H), 7.45 (dd, J=2.5, 7.5 Hz, 2H), 7.39-7.21 (m, 5H), 7.08 (7.12) (d, $\mathrm{J}=8.5 \mathrm{~Hz}, 1 \mathrm{H}), 6.98$ (6.93) (d, J=9 Hz, 1H), 5.67 (s, 1H), 5.16-5.01 (m, 3H), 4.88 (4.92) (d, J=9.5 Hz, 1H), 4.62 (4.65) (d, J=10 Hz, 1H), 4.55 (4.39) (d, J=9 Hz, 1H), 3.96 (3.91) (t, J=8 Hz, 1H), 3.83 (3.85) (d, J=6 Hz, 1H), 3.78 (3.75) (dd, J=6, $11 \mathrm{~Hz}, 1 \mathrm{H}), 1.99-1.91$ $(2.08-2.17)(\mathrm{m}, 1 \mathrm{H}), 1.99$ (br, s, 1H), 1.62-1.56 (1.88-1.82) (m, 1H), $1.42(\mathrm{~s}, 9 \mathrm{H}), 1.13$ (s, $3 \mathrm{H}), 0.96(0.80)(\mathrm{t}, \mathrm{J}=7.5 \mathrm{~Hz}, 3 \mathrm{H}), 0.76(\mathrm{~d}, \mathrm{~J}=7 \mathrm{~Hz}, 3 \mathrm{H}), 0.74(\mathrm{~d}, \mathrm{~J}=7 \mathrm{~Hz}, 3 \mathrm{H}) ;{ }^{13} \mathbf{C} \mathbf{N M R}$ (125.5 MHz, DMSO-d $\left.{ }_{6}, 80{ }^{\circ} \mathrm{C}\right) \delta 170.5,170.0,168.8$ (168.4), 168.1 (168.0), 155.7, 151.7 (151.5), 143.8 (143.6), 136.9 (136.7), 134.7 (134.6), 127.8, 127.2 (126.8), 123.2 (123.6), $121.9,114.9,83.8$ (84.0), 80.3, 70.2 (70.1), 69.1 (68.7), 65.8, 64.0, 60.0, 58.6, 57.9 (58.2), 54.2, 41.3 (40.9), 29.2, 27.6, 27.4 (27.3), 27.1, 22.4, 18.6 (18.7), 18.4, 17.4, 7.9; HRMS calculated for $\mathrm{C}_{42} \mathrm{H}_{54} \mathrm{~N}_{4} \mathrm{O}_{10}(\mathrm{M}+\mathrm{H})$ : 775.3918 , found (FAB): 775.3914. The chemical shifts in parentheses are the shifts for the minor diastereomer.

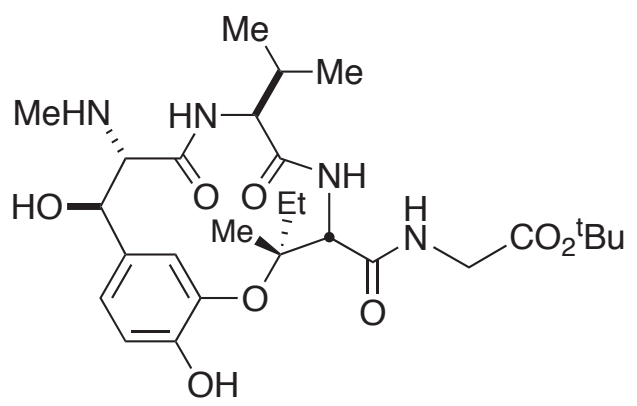

Ustiloxin D tert-butyl ester (77). Macrocycle 67 (53.0 mg, $0.0684 \mathrm{mmol}, 1$ equiv) was dissolved in methanol $(2 \mathrm{~mL})$ at room temperature. $5 \%$ Palladium on carbon $(21.8 \mathrm{mg}$, 0.0103 mmol, 0.15 equiv, purchased from Acros) was added and a hydrogen balloon was attached. After $2 \mathrm{~h}$, the hydrogen source was removed and the reaction mixture was concentrated. The crude material was purified

by directly loading onto a silica gel column. Using a gradient of $1 / 2$ hexanes/ethyl acetate then a gradient of $25 / 1$ to $10 / 1$ chloroform/methanol, ustiloxin D tert-butyl ester 77 (32.9 mg, $0.0597 \mathrm{mmol}, 87 \%)$ was obtained. $\mathrm{R}_{\mathrm{f}}=0.2$ (5/1 chloroform/methanol); IR (film) 3287 br, s, $2971 \mathrm{~m}, 2219$ w, 1748 w, 1732 w, 1643 s, 1537 s, 1369 s, 1286 s, 1222 m, 1157 s, 1132 m, 1114 m, 1062 w, 1033 w, 960 w, 888 w, 844 w, 755 m; ${ }^{1}$ H NMR (500 MHz, CD $\mathrm{OD}) \delta 7.04$ (6.95) (dd, J=2, $8 \mathrm{~Hz}, 1 \mathrm{H}), 6.96$ (6.91) (br, 1H), 6.78 (6.72) $(\mathrm{d}, \mathrm{J}=8.5 \mathrm{~Hz}, 1 \mathrm{H}), 4.65(4.54)(\mathrm{s}, 1 \mathrm{H}), 4.42(4.35)(\mathrm{d}, \mathrm{J}=8 \mathrm{~Hz}, 1 \mathrm{H}), 3.92$ (3.86) (d, J=7.5 $\mathrm{Hz}, 1 \mathrm{H}), 3.72$ (3.73) (d, J=2 Hz, 2H), 3.13 (3.08) (d, J=8 Hz, 1H), 2.23 (s, 3H), 2.04-1.92 $(2.22-2.12)(\mathrm{m}, 1 \mathrm{H}), 2.00-1.90(\mathrm{~m}, 1 \mathrm{H}), 1.94-1.84(1.64-1.56)(\mathrm{m}, 1 \mathrm{H}), 1.33(\mathrm{~s}, 9 \mathrm{H}), 1.27$ (1.16) (s, 3H), $1.03(0.90)(\mathrm{t}, \mathrm{J}=6.5 \mathrm{~Hz}, 3 \mathrm{H}), 0.73(\mathrm{~d}, \mathrm{~J}=7 \mathrm{~Hz}, 3 \mathrm{H}), 0.70(\mathrm{~d}, \mathrm{~J}=7 \mathrm{~Hz}, 3 \mathrm{H})$; ${ }^{13}$ C NMR (125.5 MHz, CD $\left.\mathrm{OD}\right) \delta 182.0,174.3,171.9,171.8$ (171.7), 169.9, 152.1 (152.0), 143.6 (143.9), 133.6 (133.9), 124.7, 123.2, 118.6 (118.0), 95.9, 85.7, 85.4, 82.8, 75.1, 72.2, 61.2, 60.7, 42.9, 34.4 (34.6), 29.6 (29.9), 28.3, 19.4, 18.4, 8.8 (8.5); HRMS 
calculated for $\mathrm{C}_{27} \mathrm{H}_{42} \mathrm{~N}_{4} \mathrm{O}_{8}(\mathrm{M}+\mathrm{H})$ : 551.3081, found (FAB): 551.3076. The chemical shifts in parentheses are the shifts for the minor diastereomer.

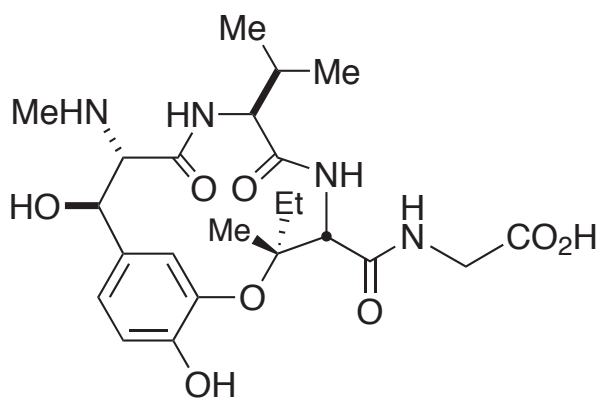

Ustiloxin D (1). Ustiloxin D tert-butyl ester 77 (7.4 mg, $0.0134 \mathrm{mmol}, 1$ equiv) was dissolved in THF $(1.4 \mathrm{~mL})$ at room temperature. Lithium hydroxide (1 N solution in water) $(1.4 \mathrm{~mL}, 0.14$ mmol, 10 equiv) was added and the reaction was stirred for $5 \mathrm{~h}$. The reaction mixture was acidified by the addition of $1 \mathrm{~N} \mathrm{HCl}$ (about 20 drops) and the solvent was removed under reduced pressure. The crude product was purified using a Sep-Pak C18. The Sep-Pak column was activated prior to use by pre-treating with $5 \mathrm{~mL}$ of methanol followed by $15 \mathrm{~mL}$ of water. The crude compound was loaded using water. Water $(3 \mathrm{~mL})$ was run to elute all the inorganic salts. The desired compound ustiloxin D 1 (6.2 mg, $0.0125 \mathrm{mmol}, 94 \%)$ was eluted using 1/1 water/methanol (6 mL). Analytical reverse phase HPLC indicated that the product was a 2:1 mixture of ustiloxin D 1: C2epi-ustiloxin D 59. Rt $=7.2 \mathrm{~min}$ (minor isomer), $9.6 \mathrm{~min}$ (major isomer) Waters Xterra M8 phenyl ( $1 \mathrm{~mL} / \mathrm{min}, 5 \%$ acetonitrile/water containing $0.05 \%$ trifluoroacetic acid). These were separated preparatively using conditions identical to the analytical run. $\mathrm{R}_{\mathrm{f}}=0.5$ (1/2 chloroform/methanol); $[\alpha]^{27.5}=-49.7^{\circ}\left(\mathrm{c} \quad 0.35, \mathrm{H}_{2} \mathrm{O}\right)$, natural ustiloxin $\mathrm{D}$ : $[\alpha]^{\text {po }}=-48.8^{\circ}\left(\mathrm{c} 0.5 \mathrm{H}_{2} \mathrm{O}\right)$; IR $(\mathrm{KBr}) 3421 \mathrm{br}, \mathrm{s}, 2930 \mathrm{w}, 1654 \mathrm{~m}, 1389 \mathrm{w}, 1070 \mathrm{br}, \mathrm{s}, 800$ w; ${ }^{1} \mathbf{H}$ NMR $\left(500 \mathrm{MHz}, \mathrm{D}_{2} \mathrm{O}\right) \delta 7.01$ (dd, J=2, $\left.8.5 \mathrm{~Hz}, 1 \mathrm{H}\right), 6.90(\mathrm{~d}, \mathrm{~J}=8 \mathrm{~Hz}, 1 \mathrm{H}), 6.84$ (br, s, 1H), 4.60 (d, J=9.5 Hz, 1H), 3.90 (d, J=10 Hz, 1H), 3.74 (d, J=9 Hz, 1H), 3.67 (s, 2H), 2.57 (s, 3H), $1.97(\mathrm{dq}, \mathrm{J}=7,21 \mathrm{~Hz}, 1 \mathrm{H}), 1.69(\mathrm{~m}, 1 \mathrm{H}), 1.53$ (s, 3H), 1,52 (dq, J=7.3, $21 \mathrm{~Hz}, 1 \mathrm{H}), 0.91(\mathrm{t}, \mathrm{J}=7.5 \mathrm{~Hz}, 3 \mathrm{H}), 0.67(\mathrm{~d}, \mathrm{~J}=7 \mathrm{~Hz}, 3 \mathrm{H}), 0.59$ (d, J=7 Hz, 3H); ${ }^{13} \mathbf{C}$ NMR $\left(125.5 \mathrm{MHz}, \mathrm{D}_{2} \mathrm{O}\right) \delta 171.2,171.1,166.3,151.0,142.7,130.7,124.0,122.7,119.1,86.0$, 73.1, 69.0, 60.2, 59.5, 32.4, 29.1, 21.3, 18.5, 18.1, 8.1; HRMS calculated for $\mathrm{C}_{23} \mathrm{H}_{34} \mathrm{~N}_{4} \mathrm{O}_{8}$ $(\mathrm{M}+\mathrm{H}):$ 495.2455, found (FAB): 495.2463 . 
page S34
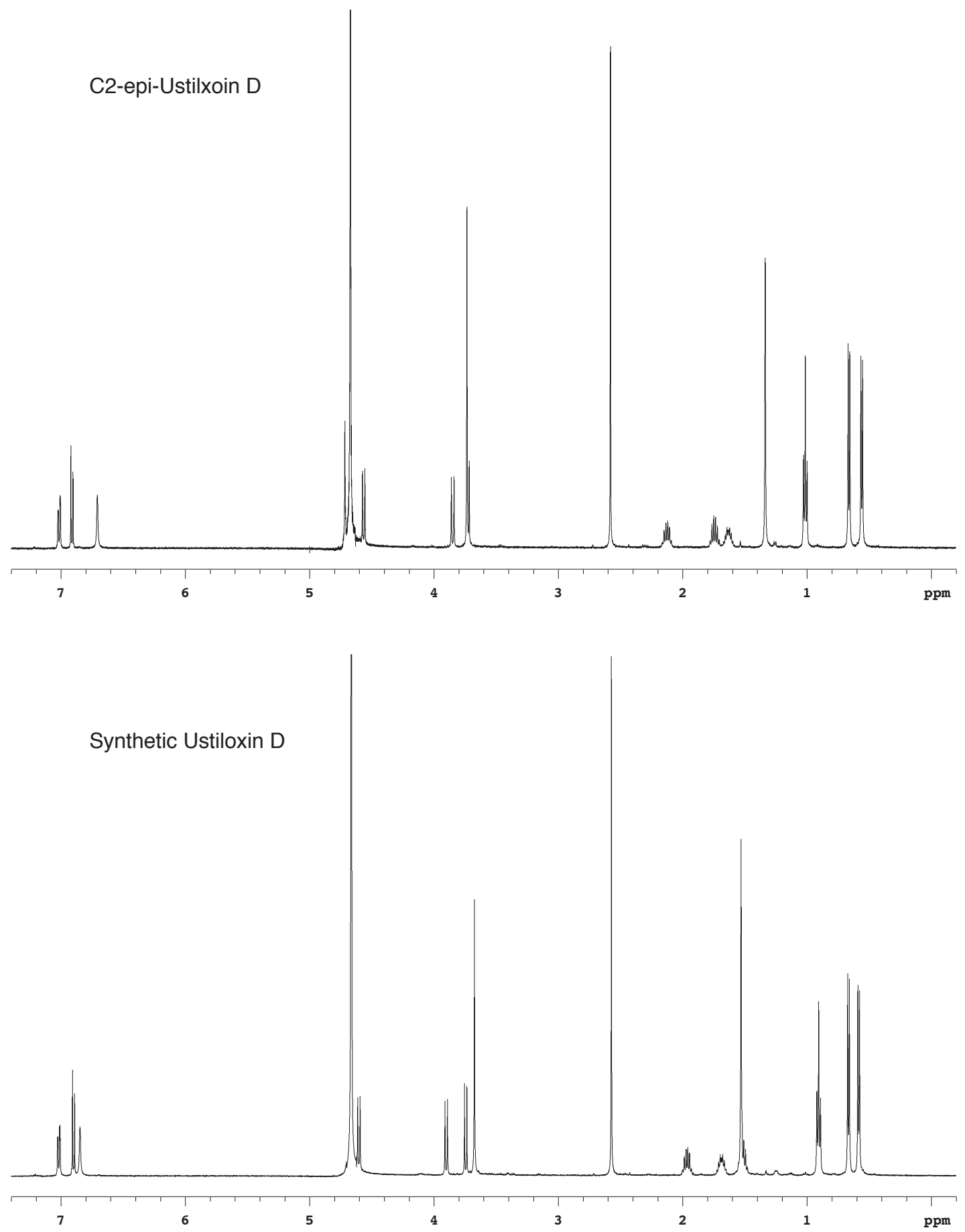
page S35
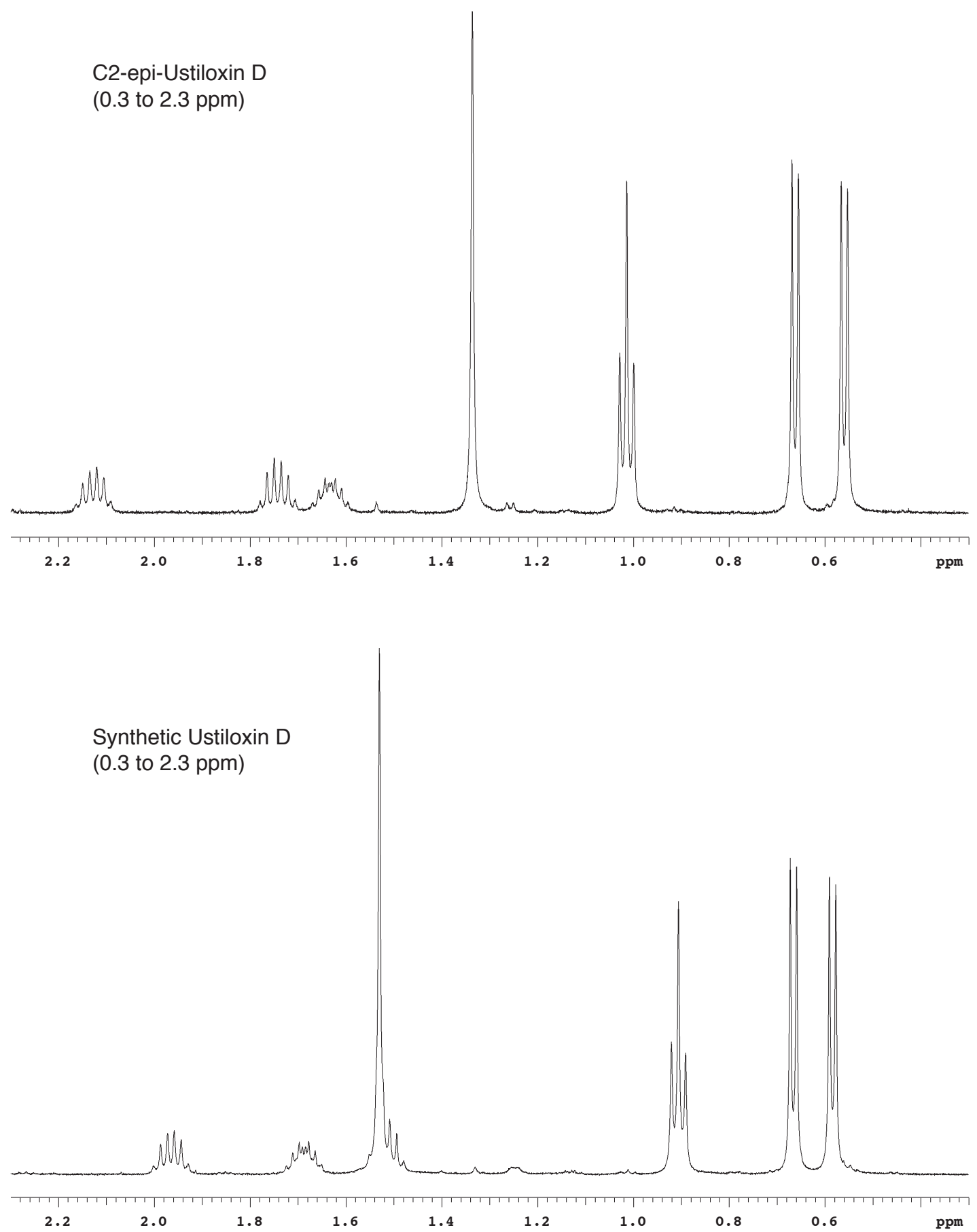
page S36
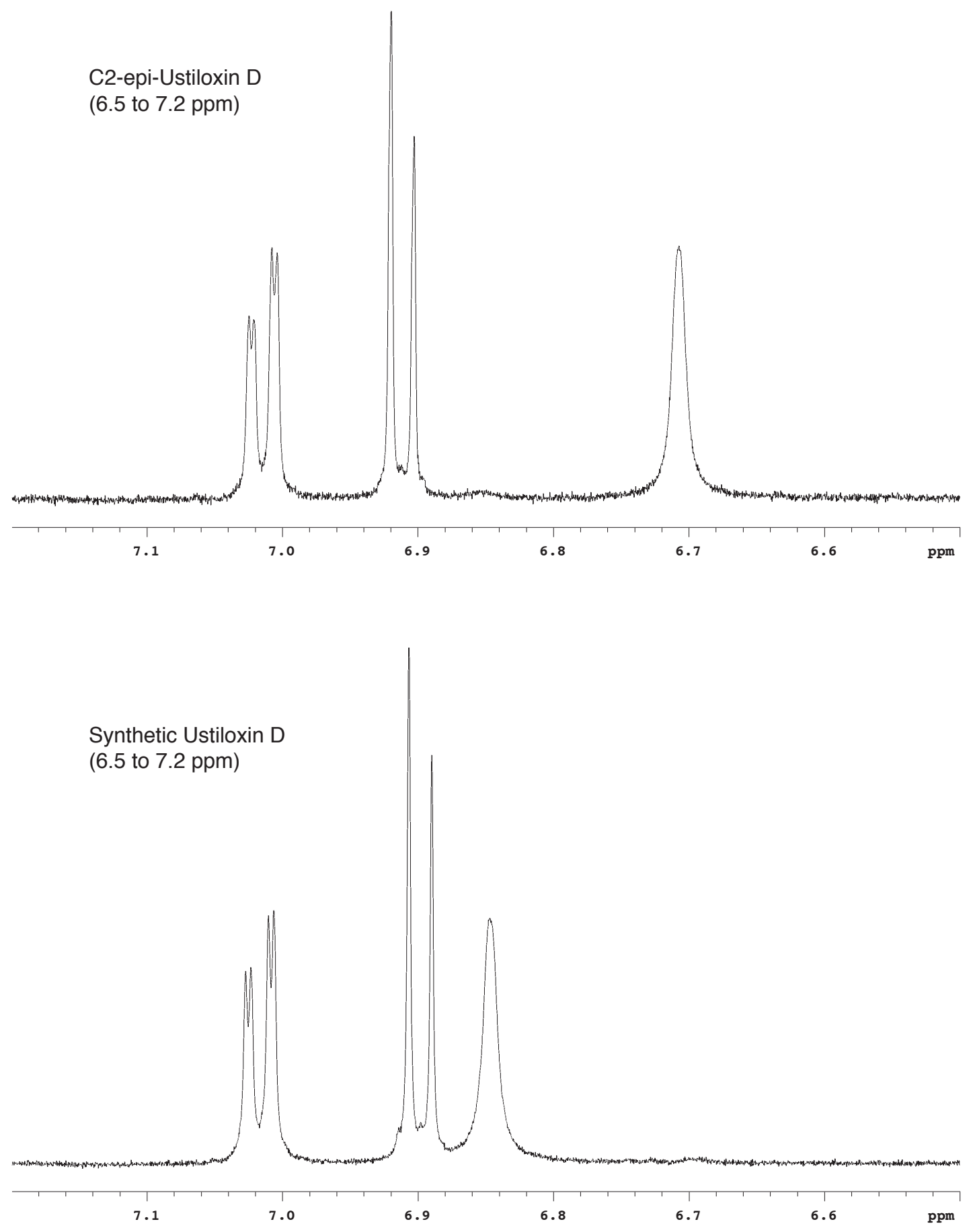


\section{page S37}

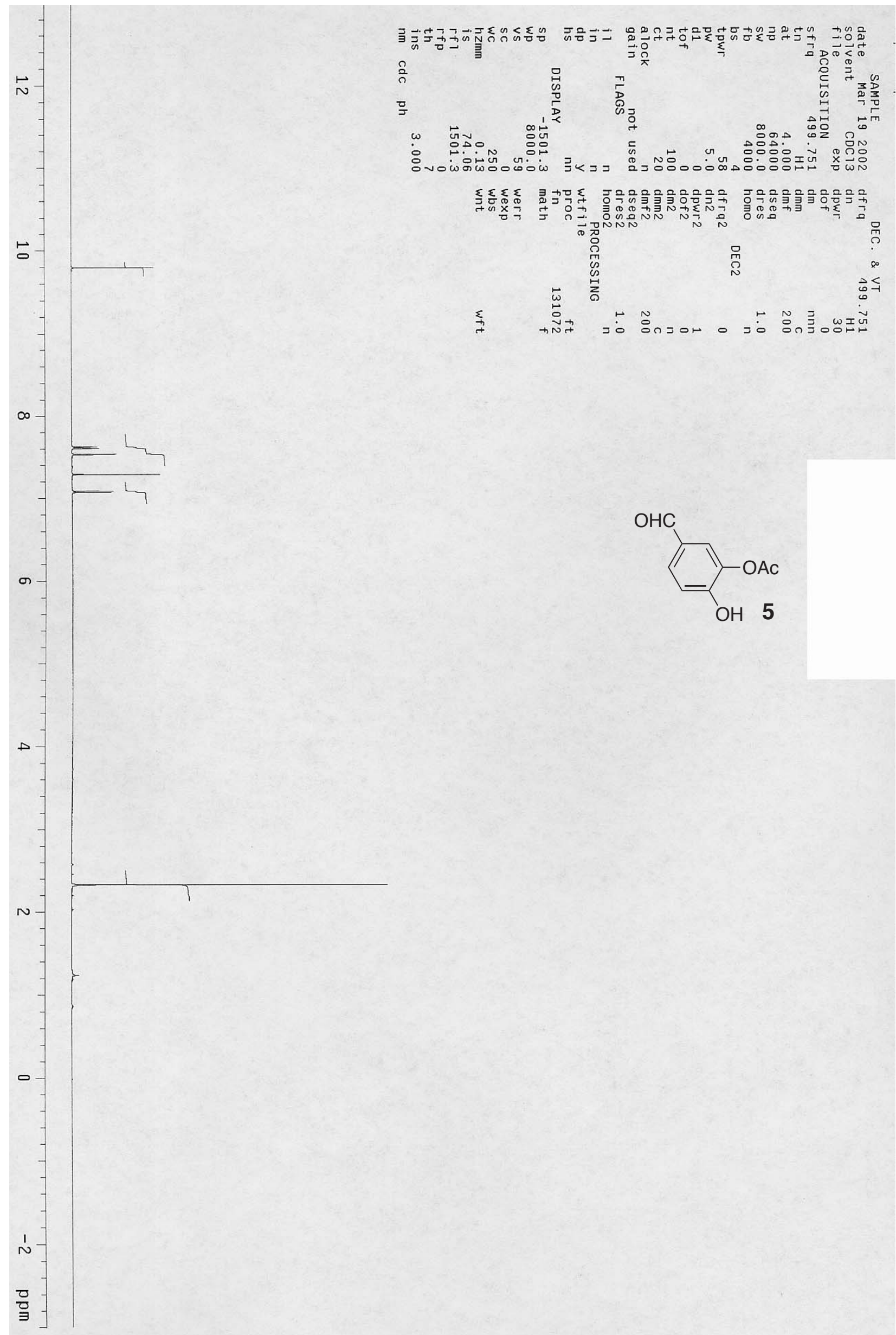


page $\mathrm{S} 38$

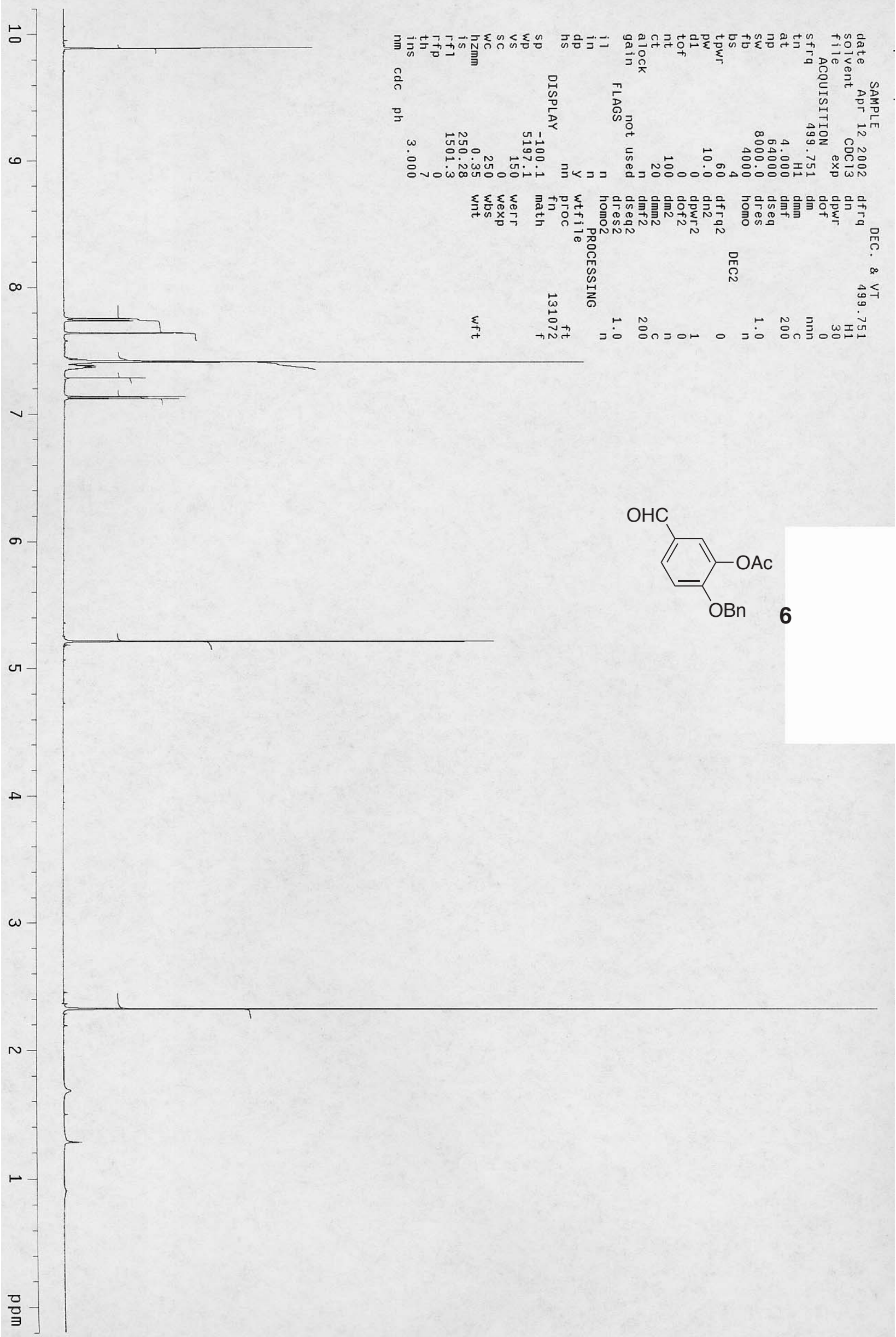




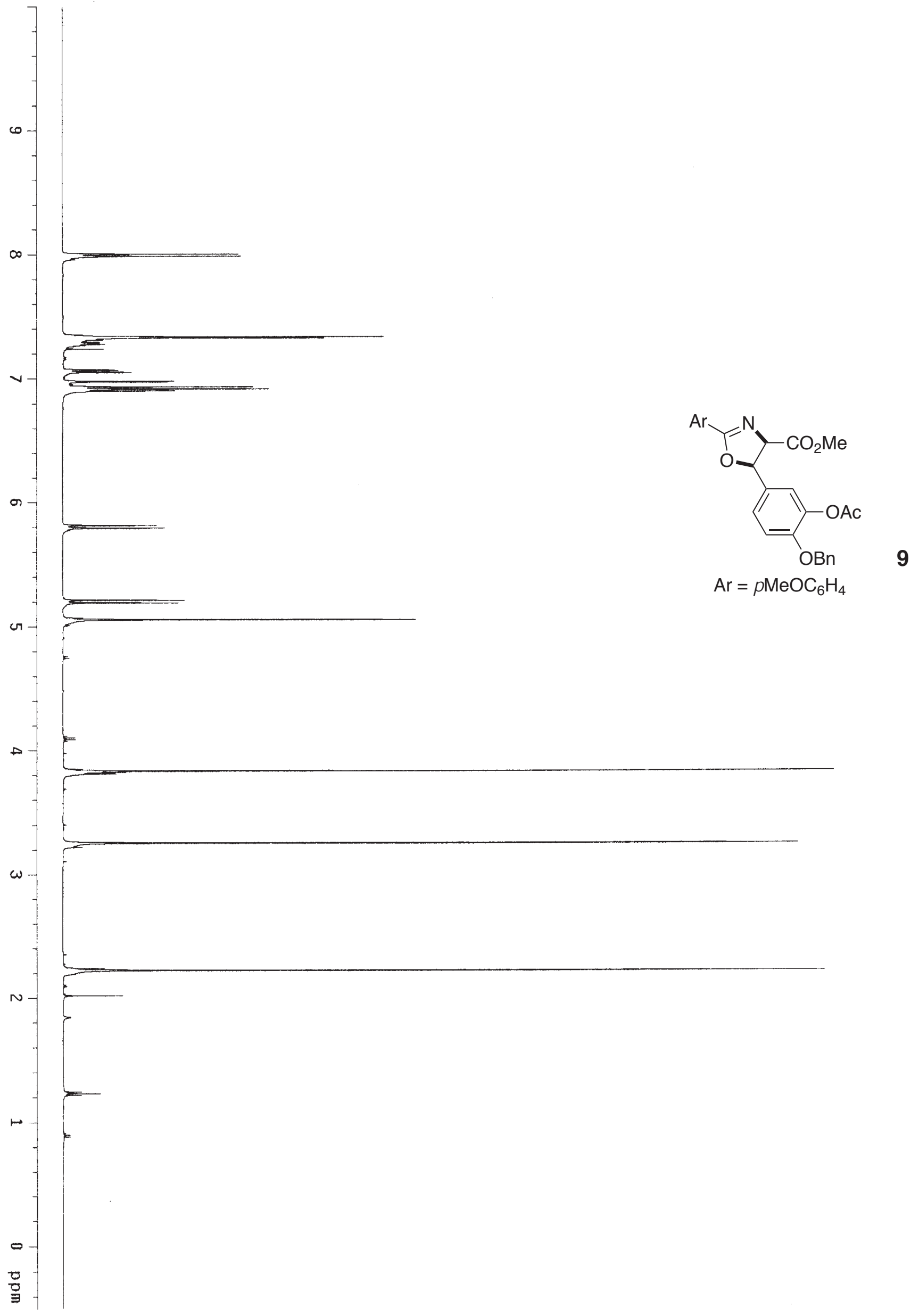


page $\mathrm{S} 40$

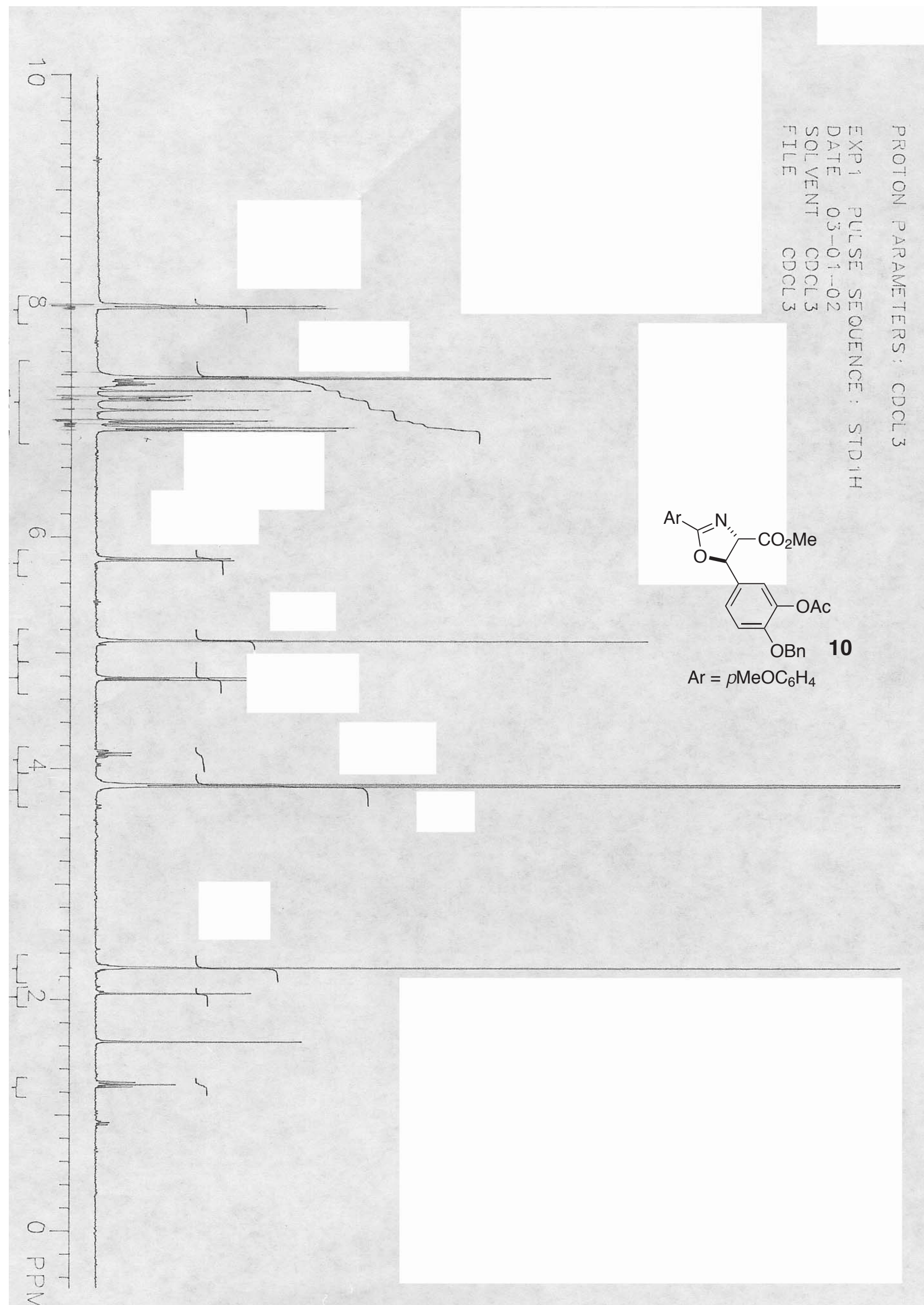


page $\mathrm{S} 41$

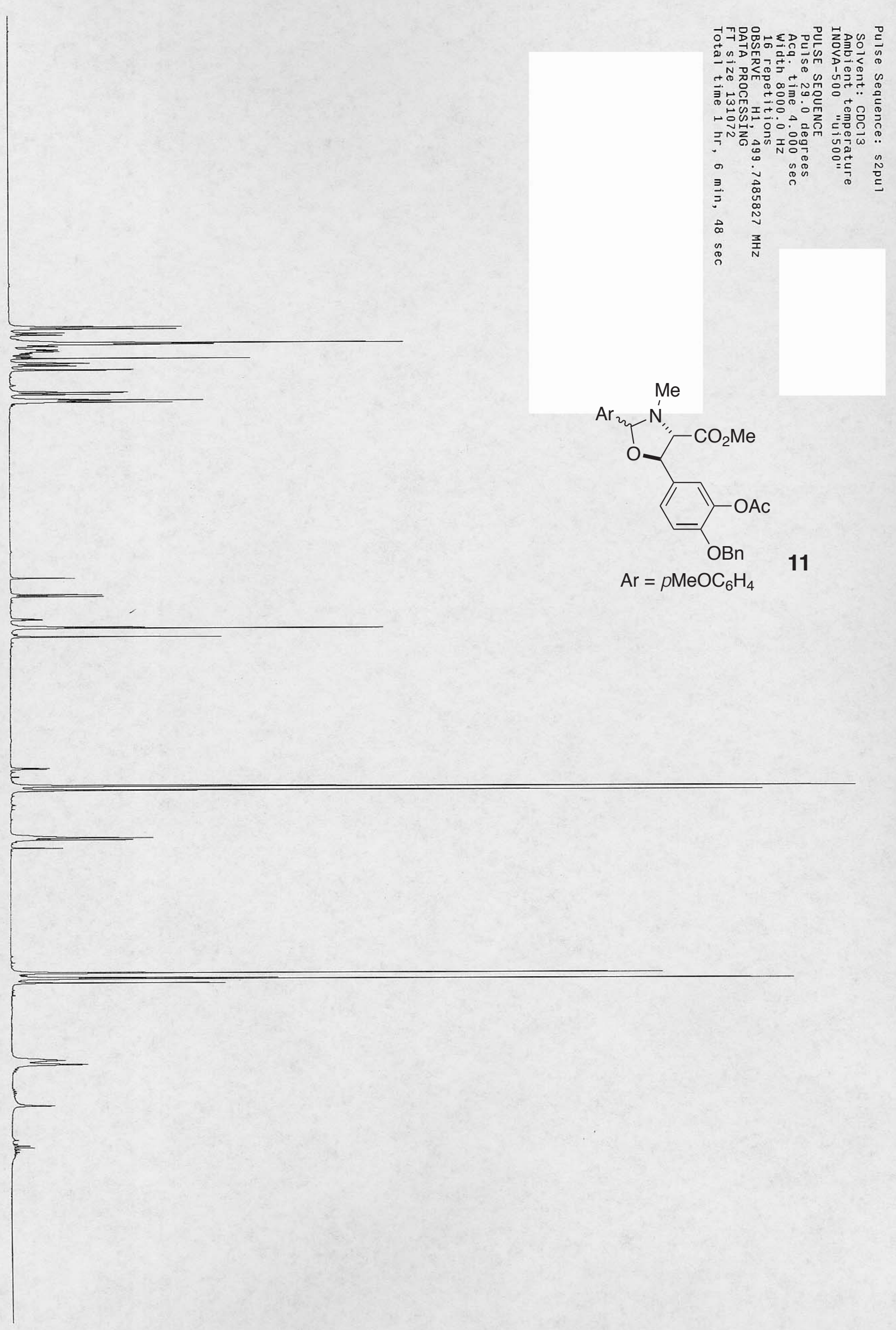


page $\mathrm{S} 42$

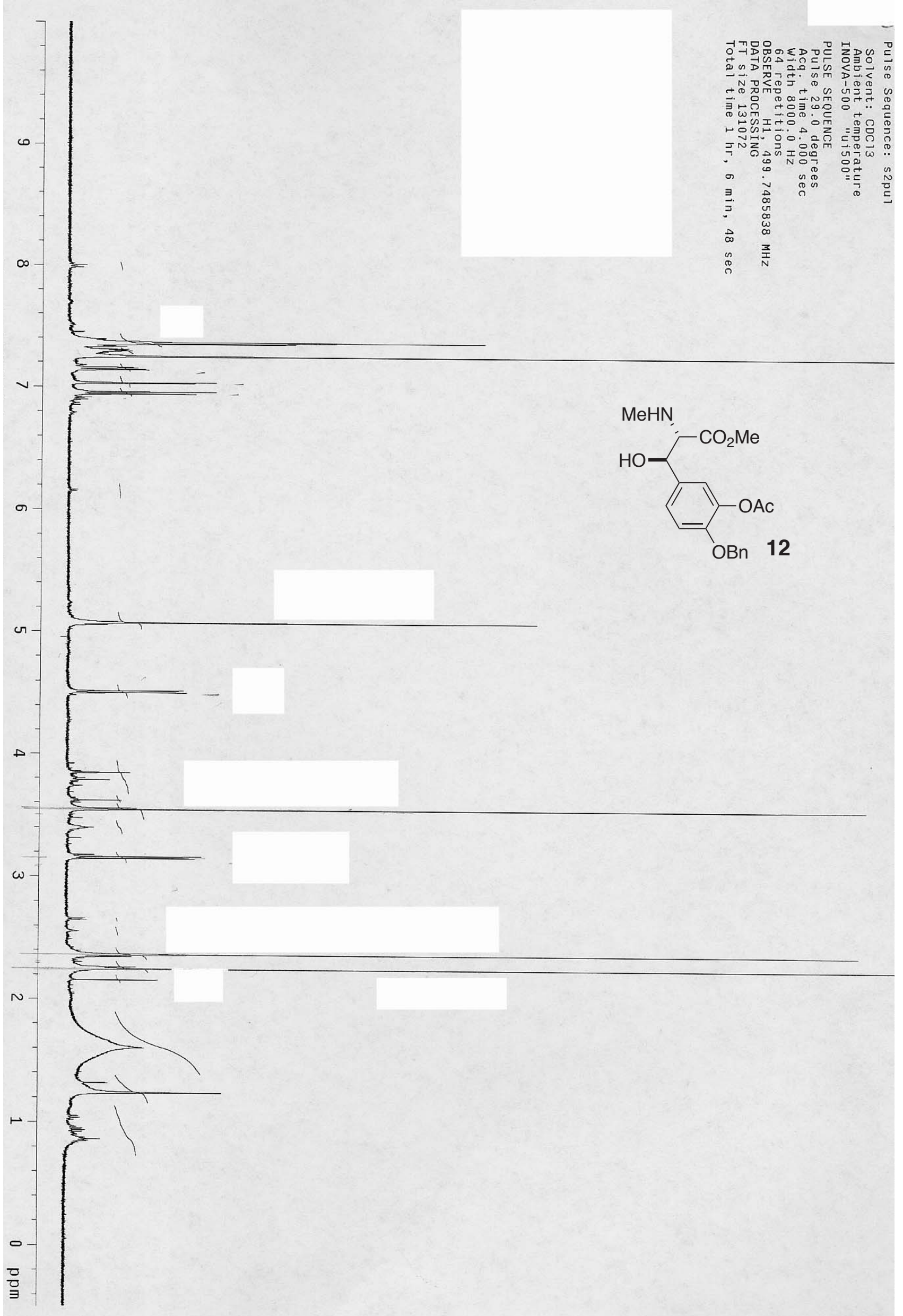


page $S 43$

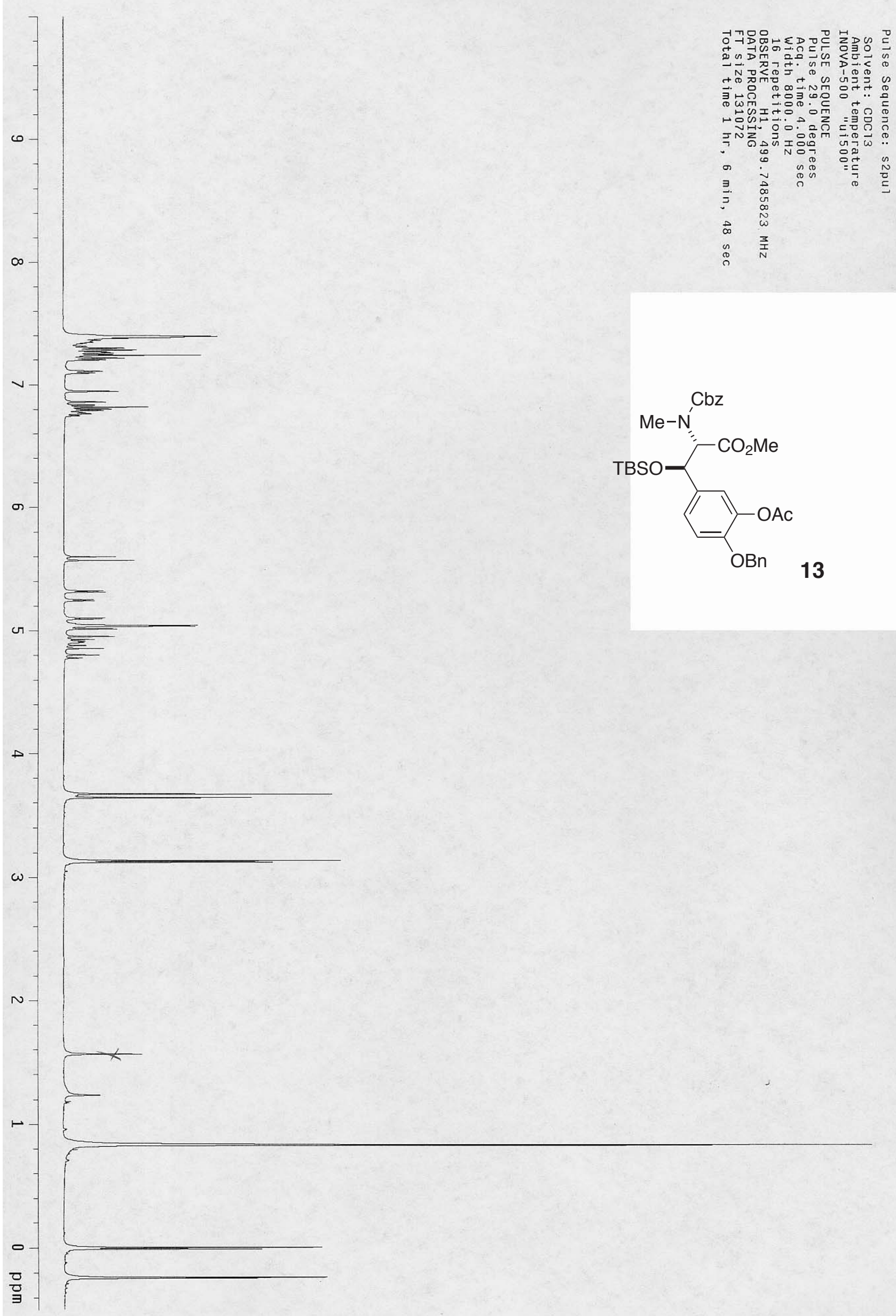


page $\mathrm{S} 44$

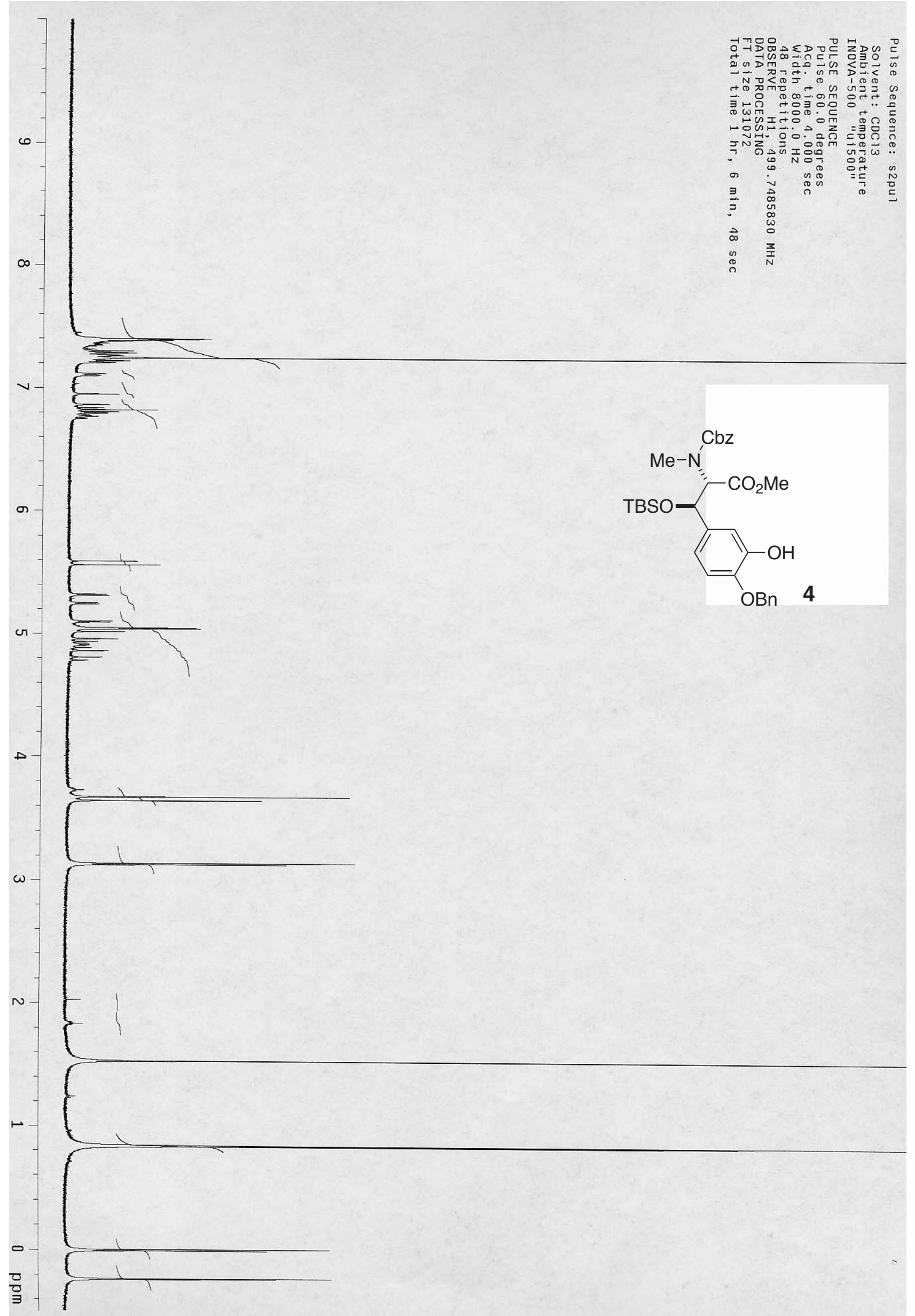


page $S 45$
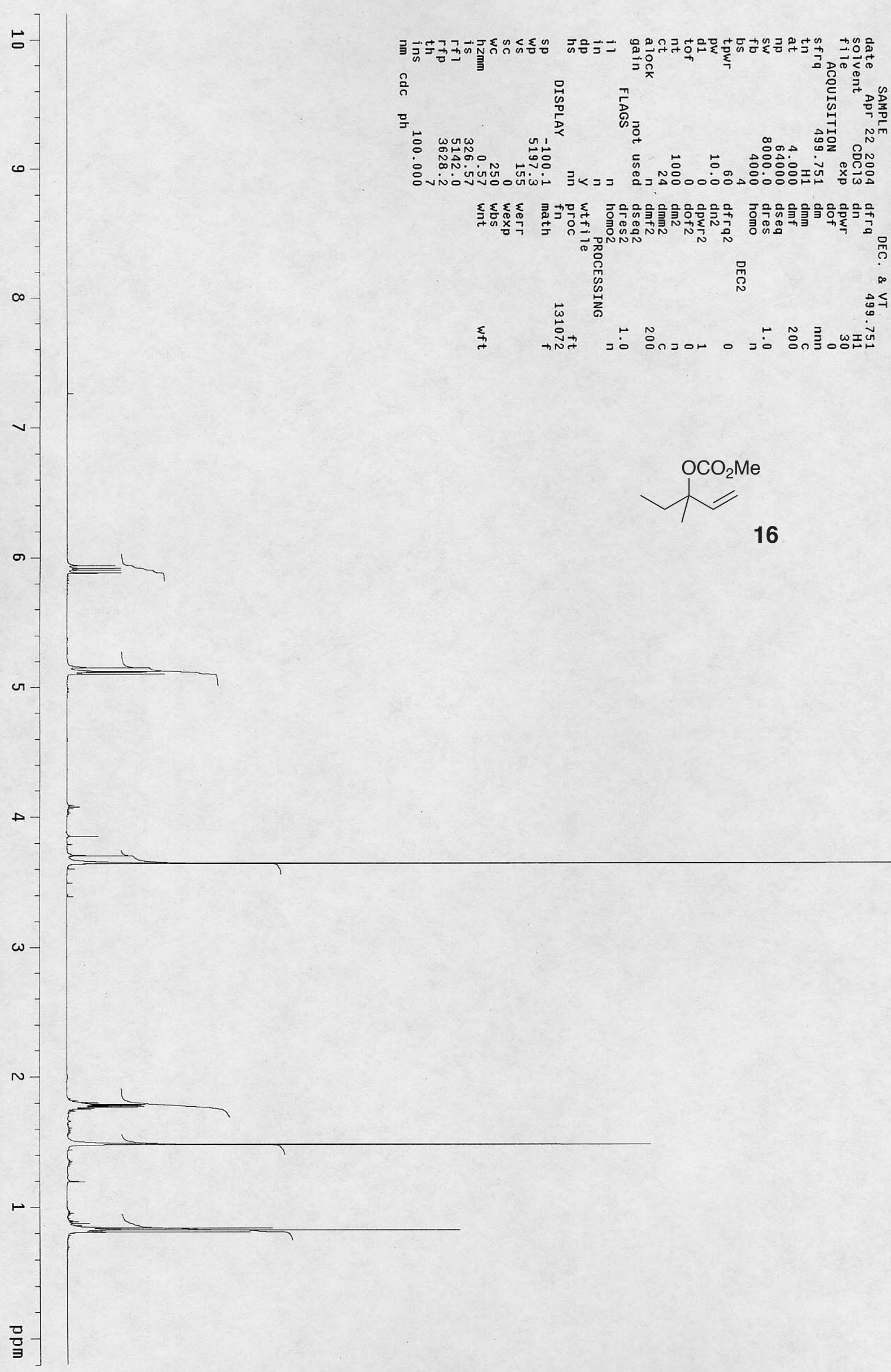
page $\mathrm{S} 46$
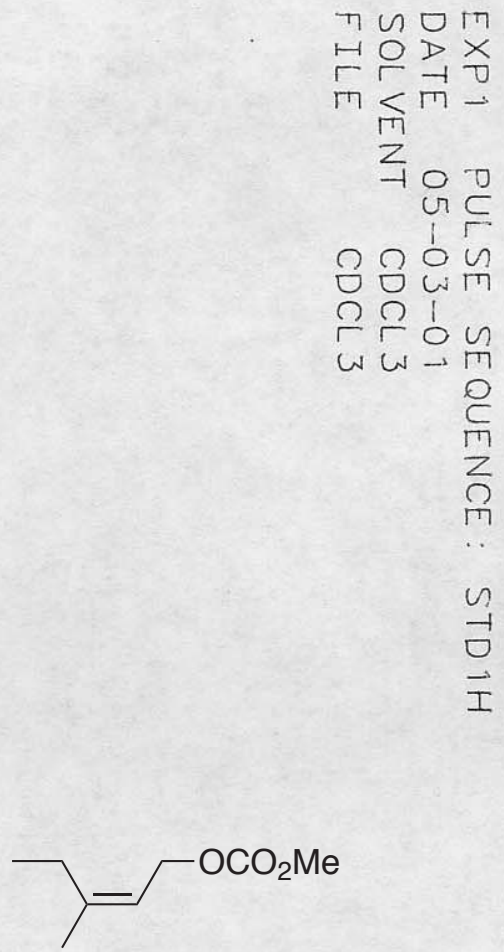
page $S 47$

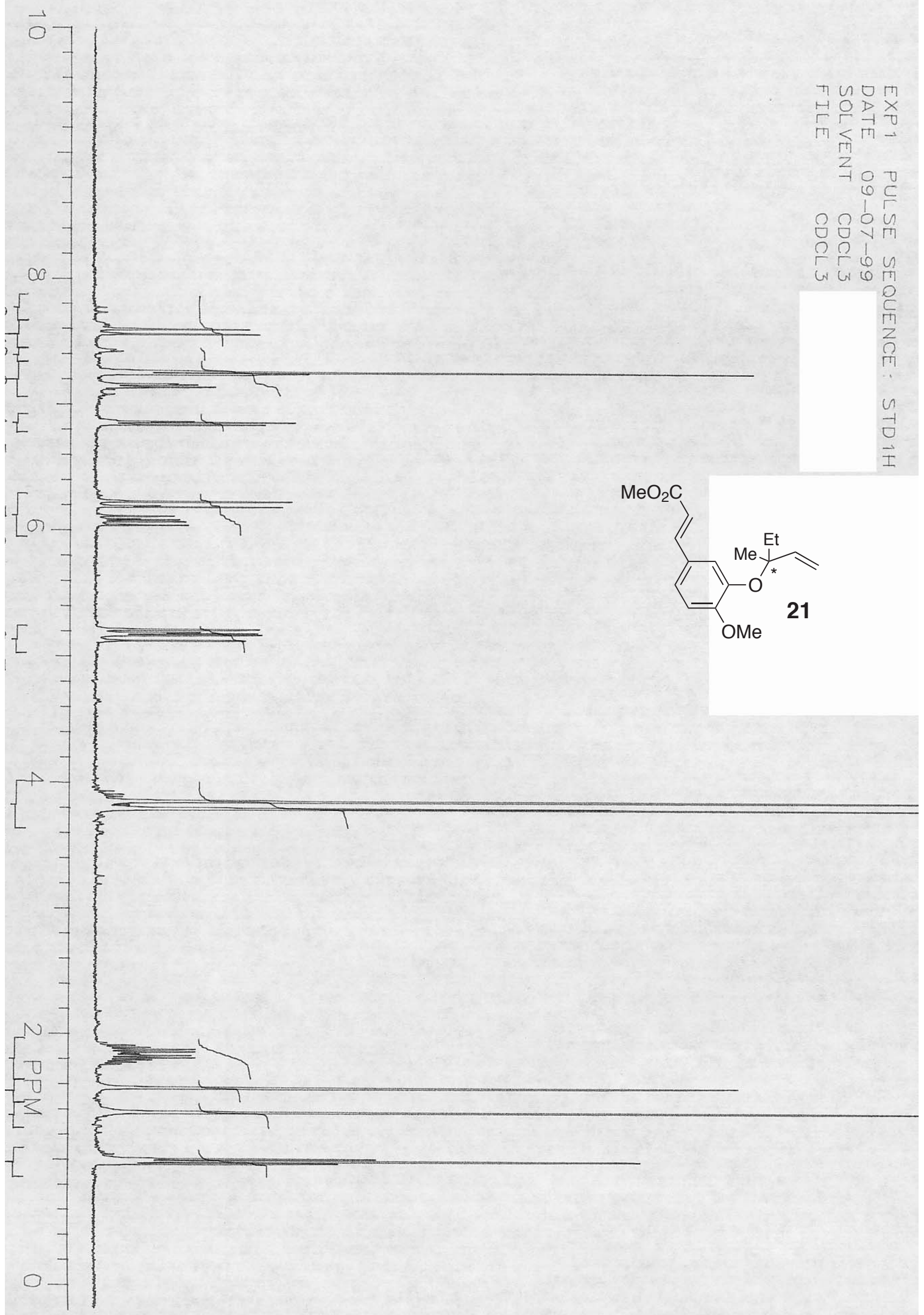


page $\mathrm{S} 48$

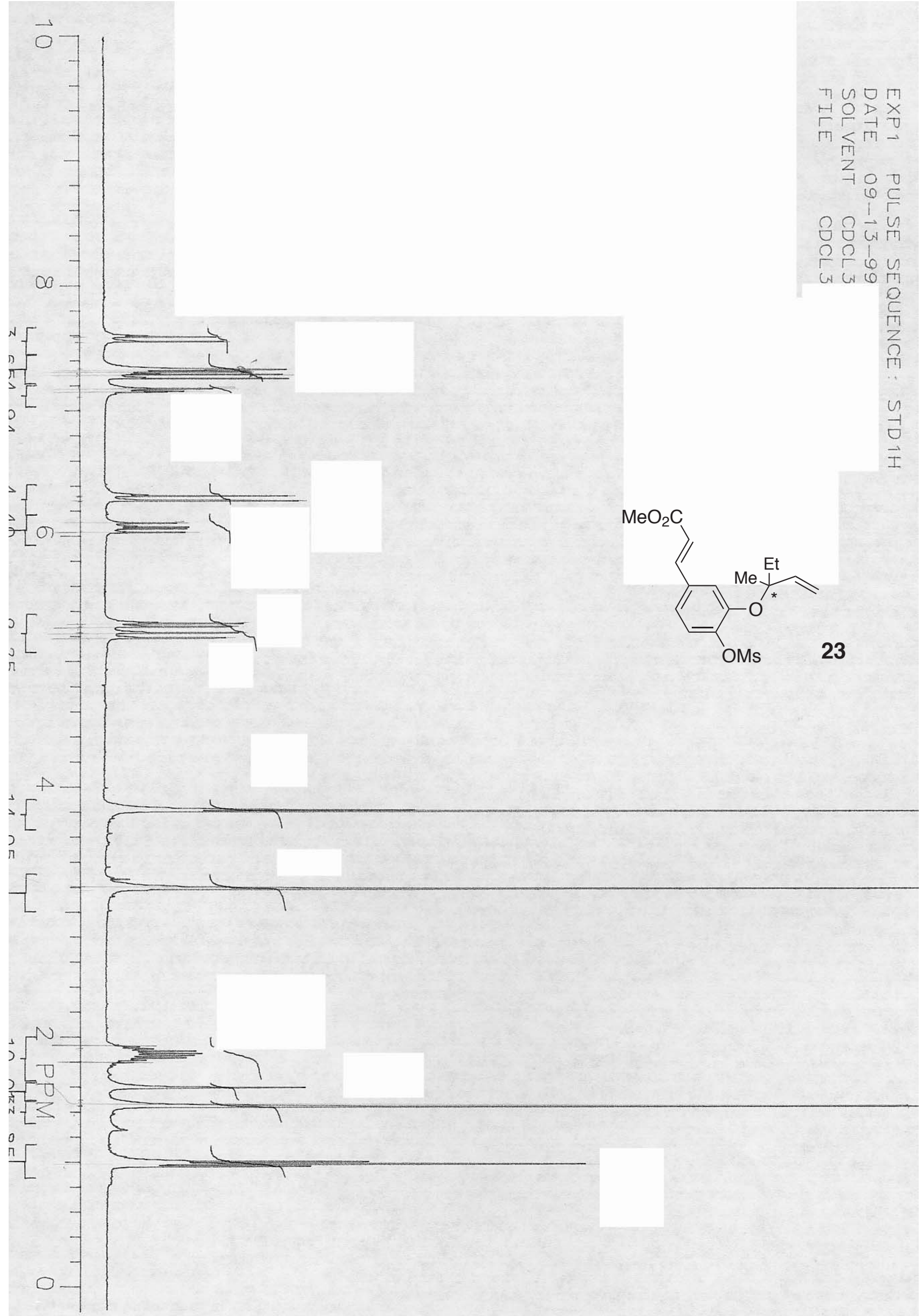


page $S 49$

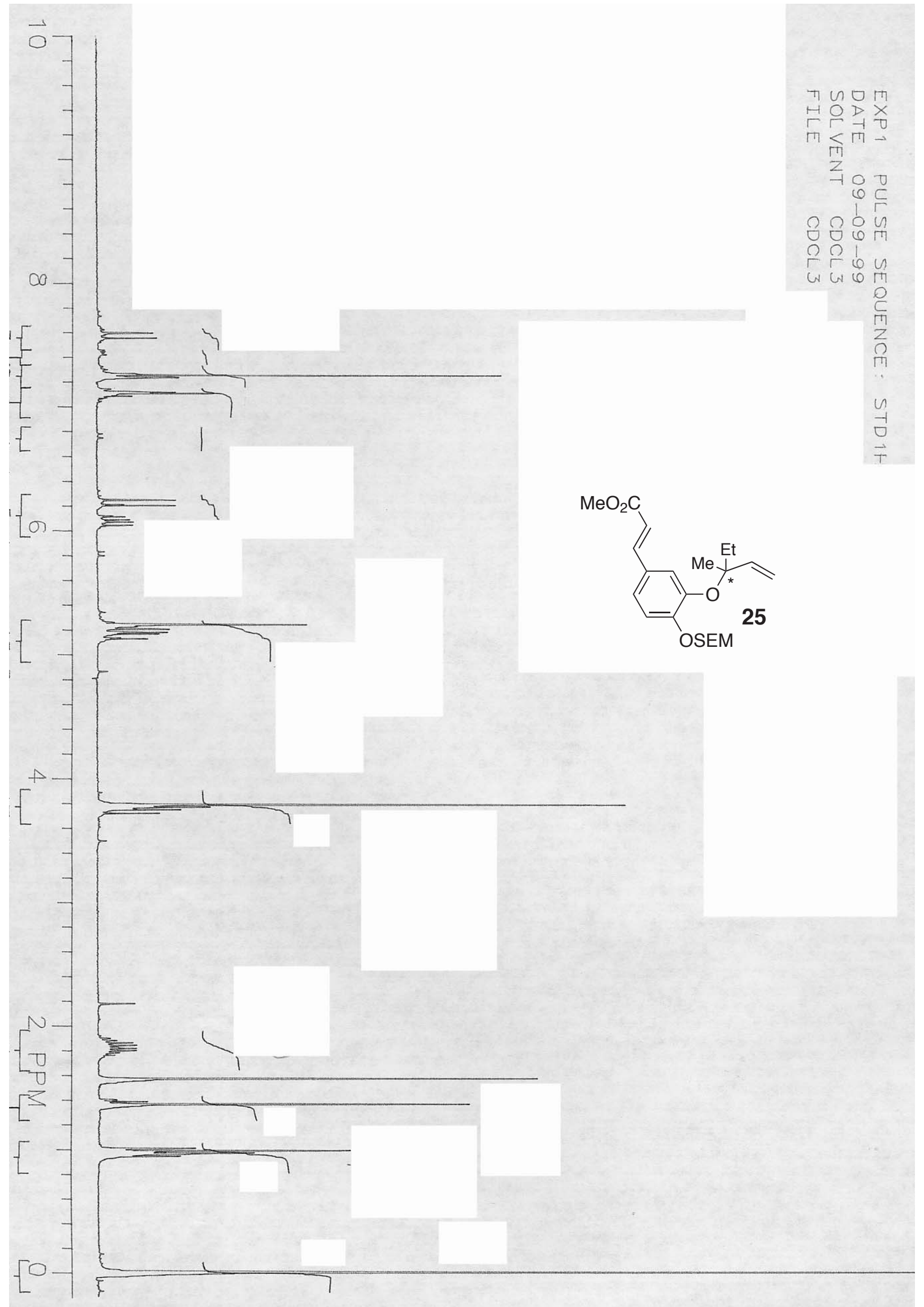


page $\mathrm{S} 50$

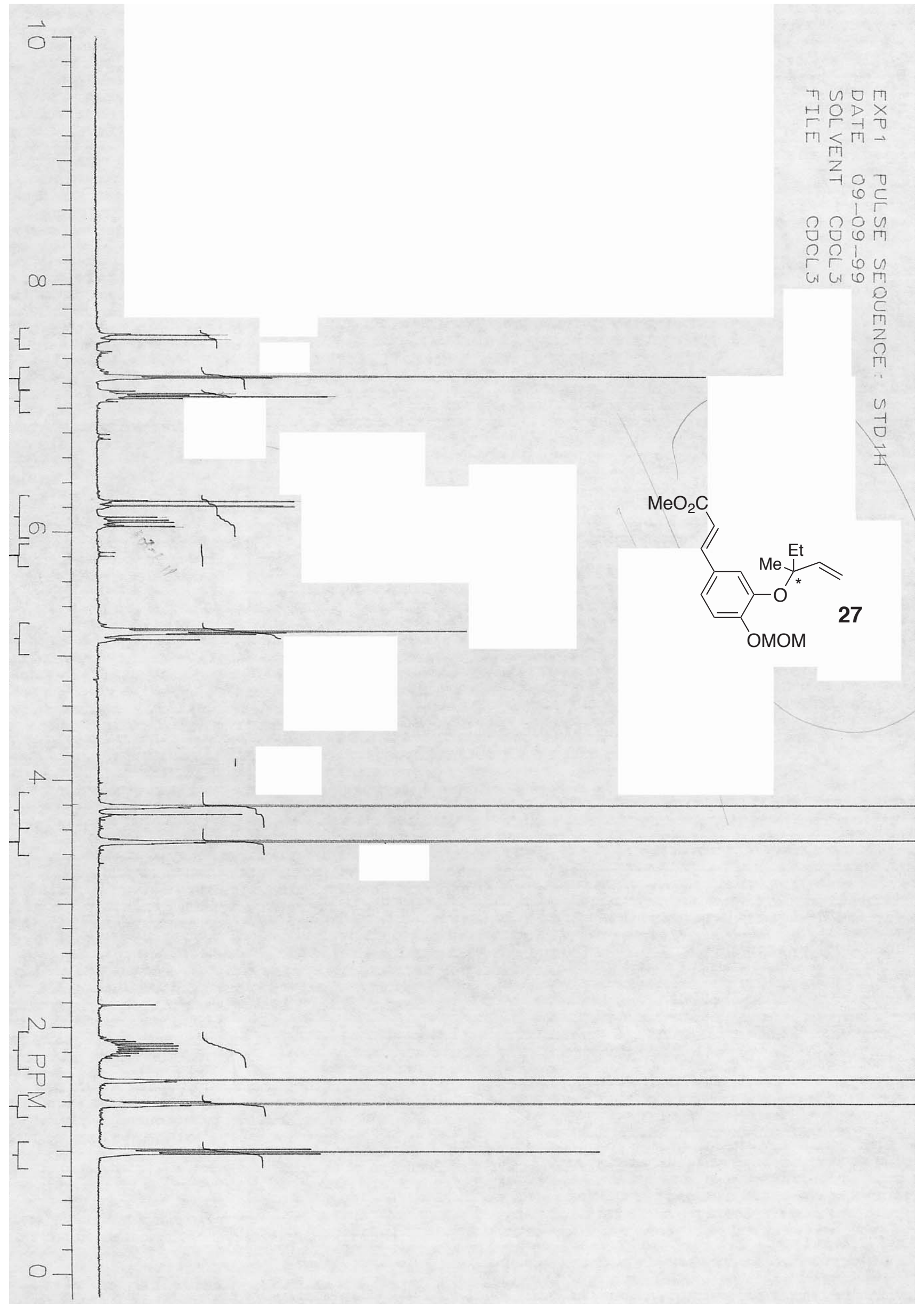


page $S 51$

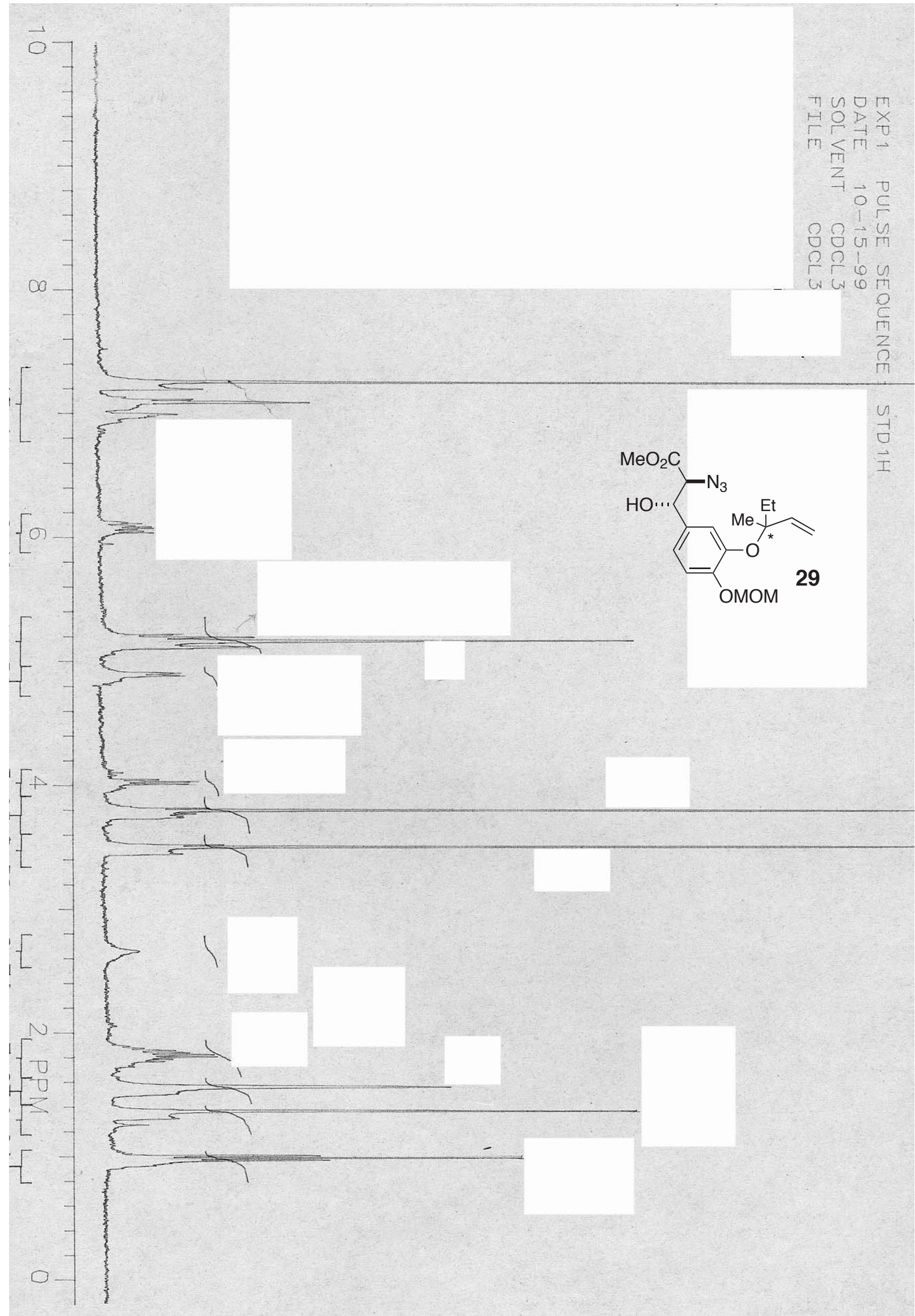




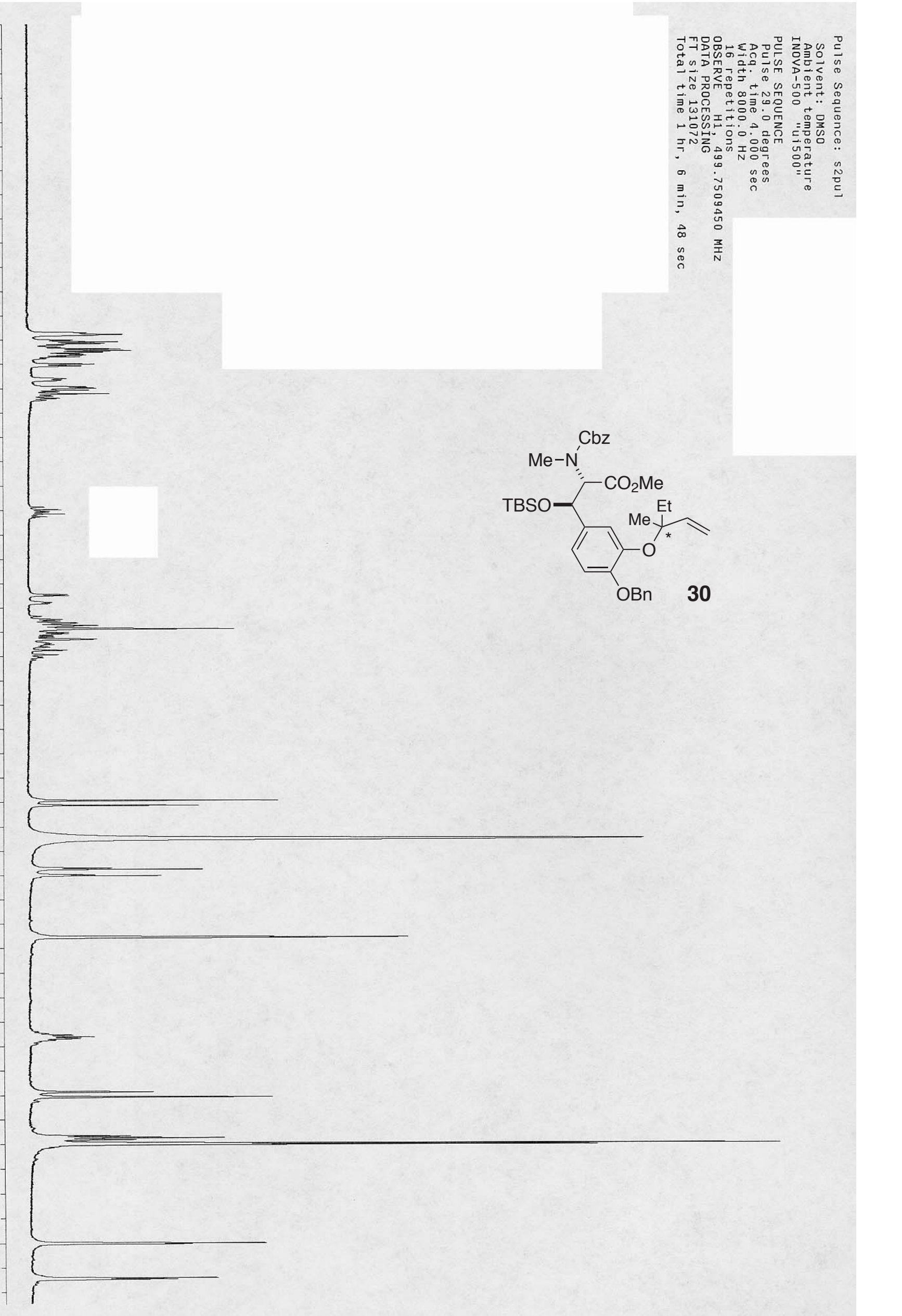




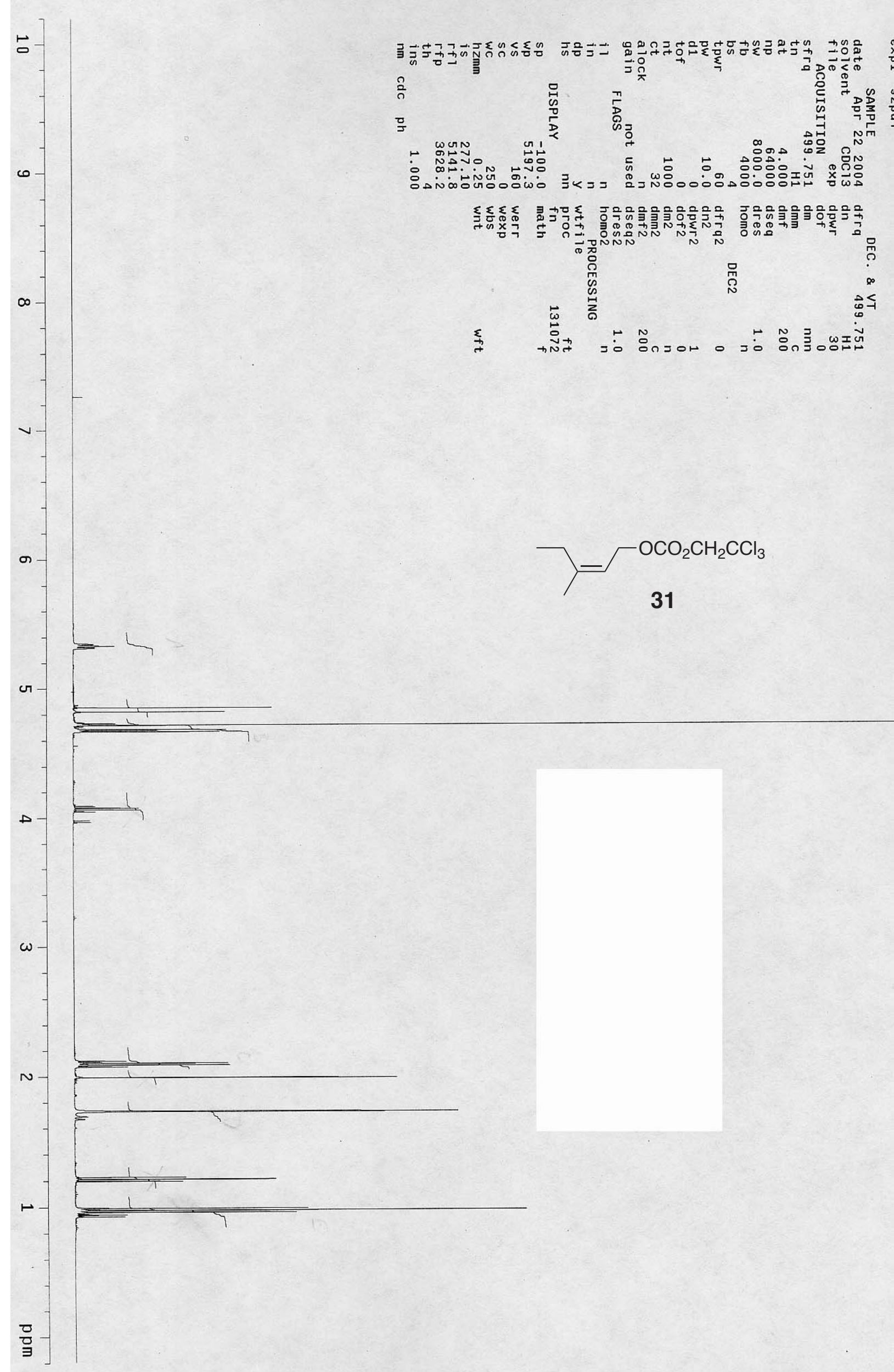




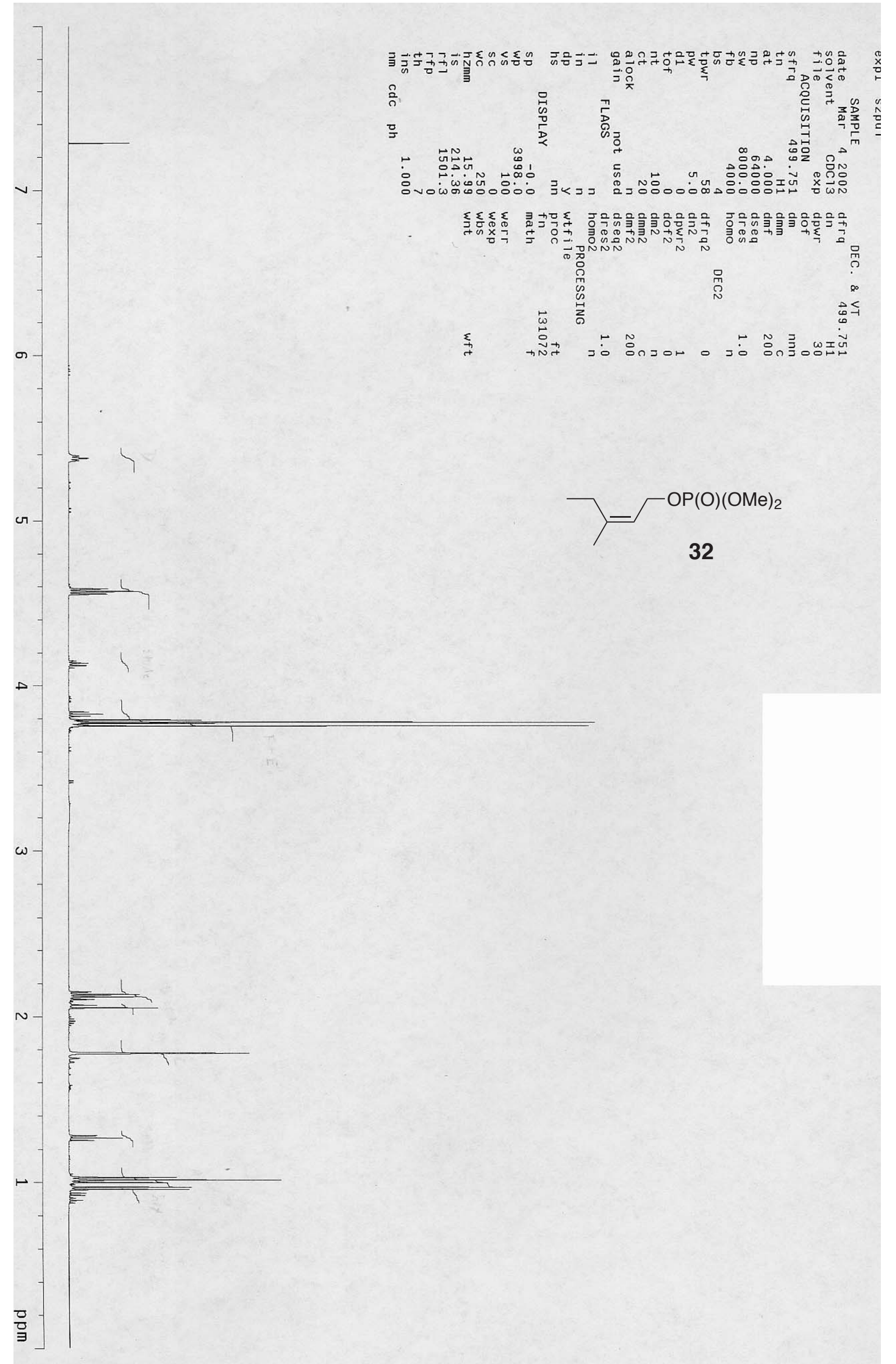




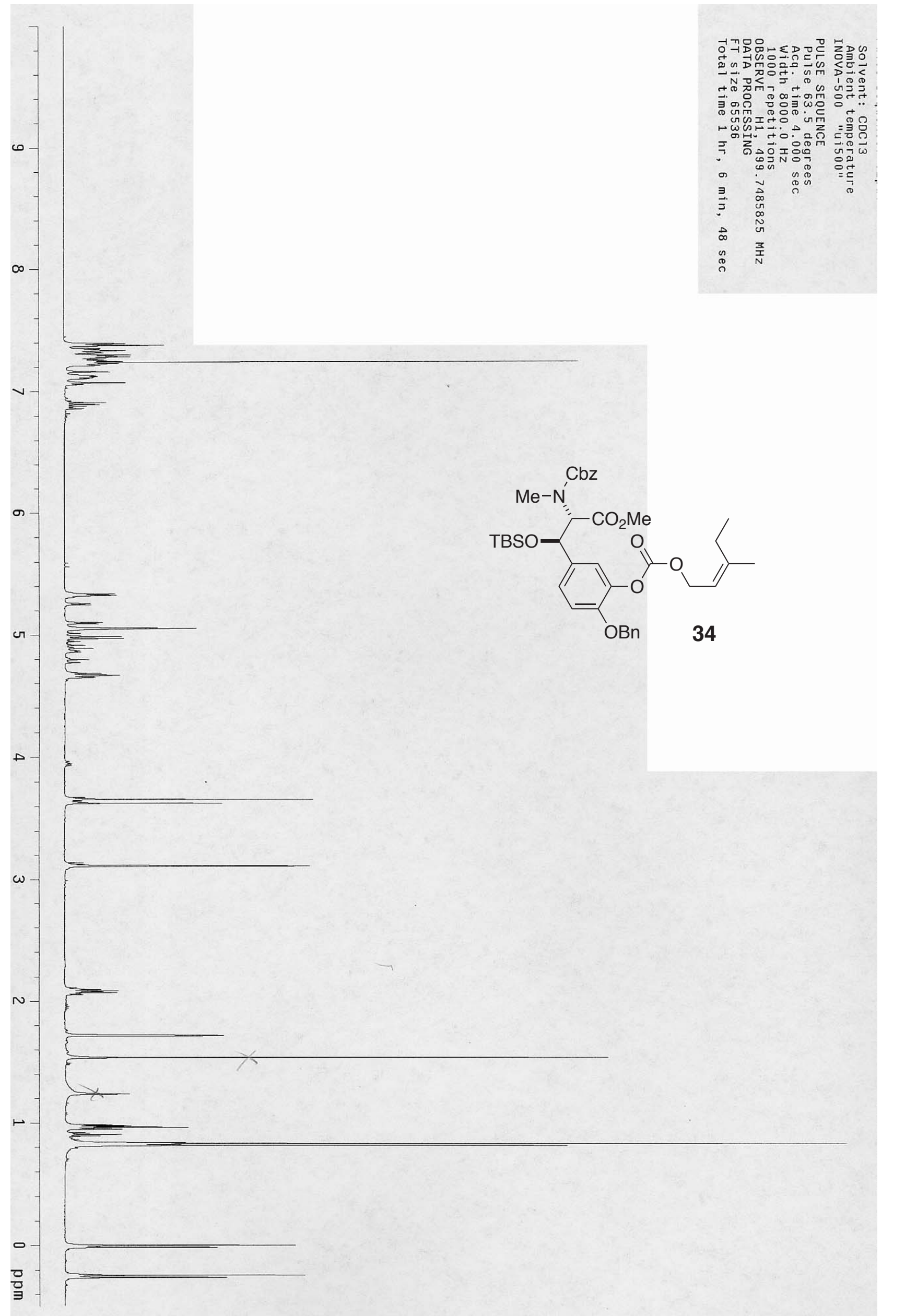


page S56

$\infty$
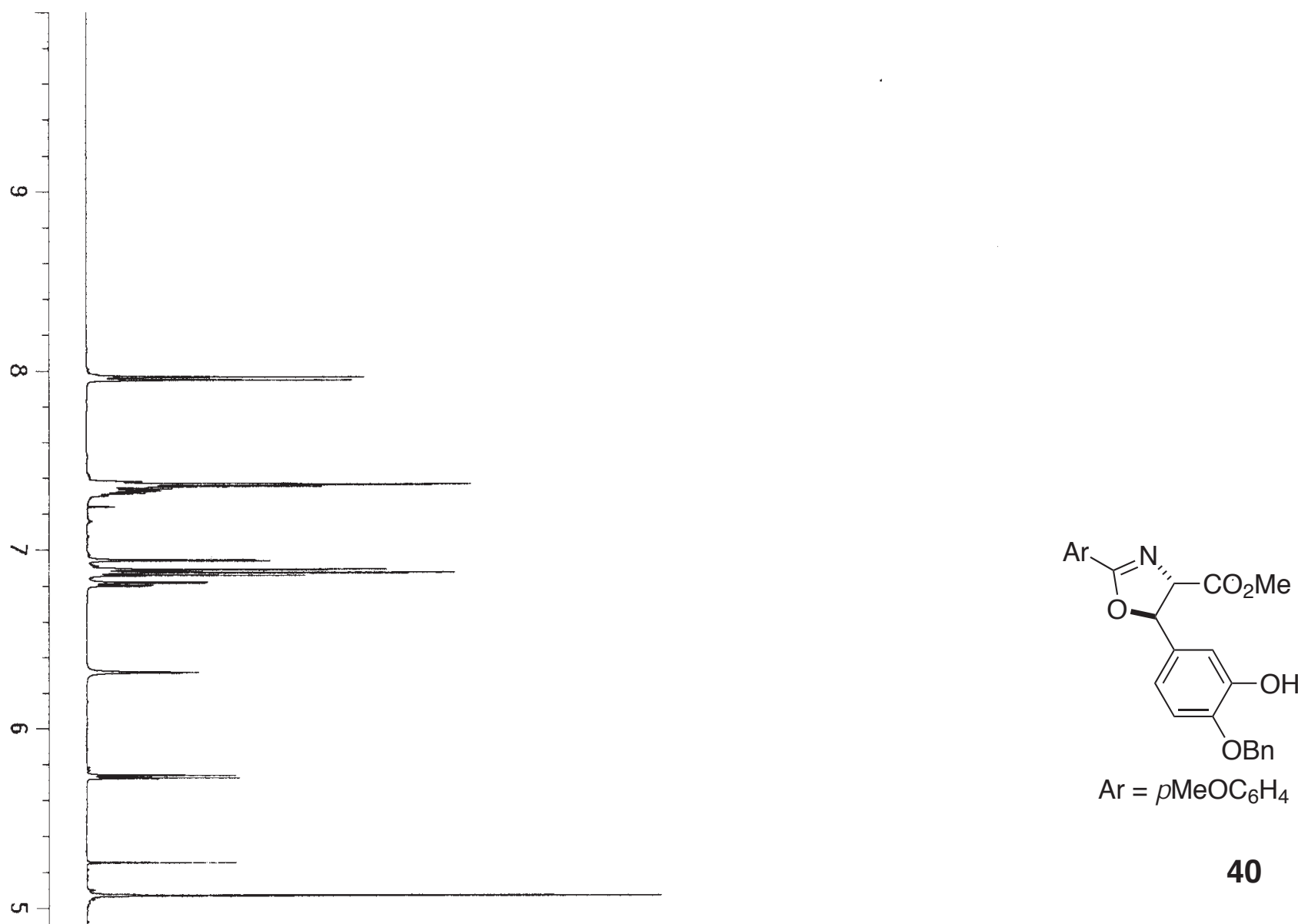

40 


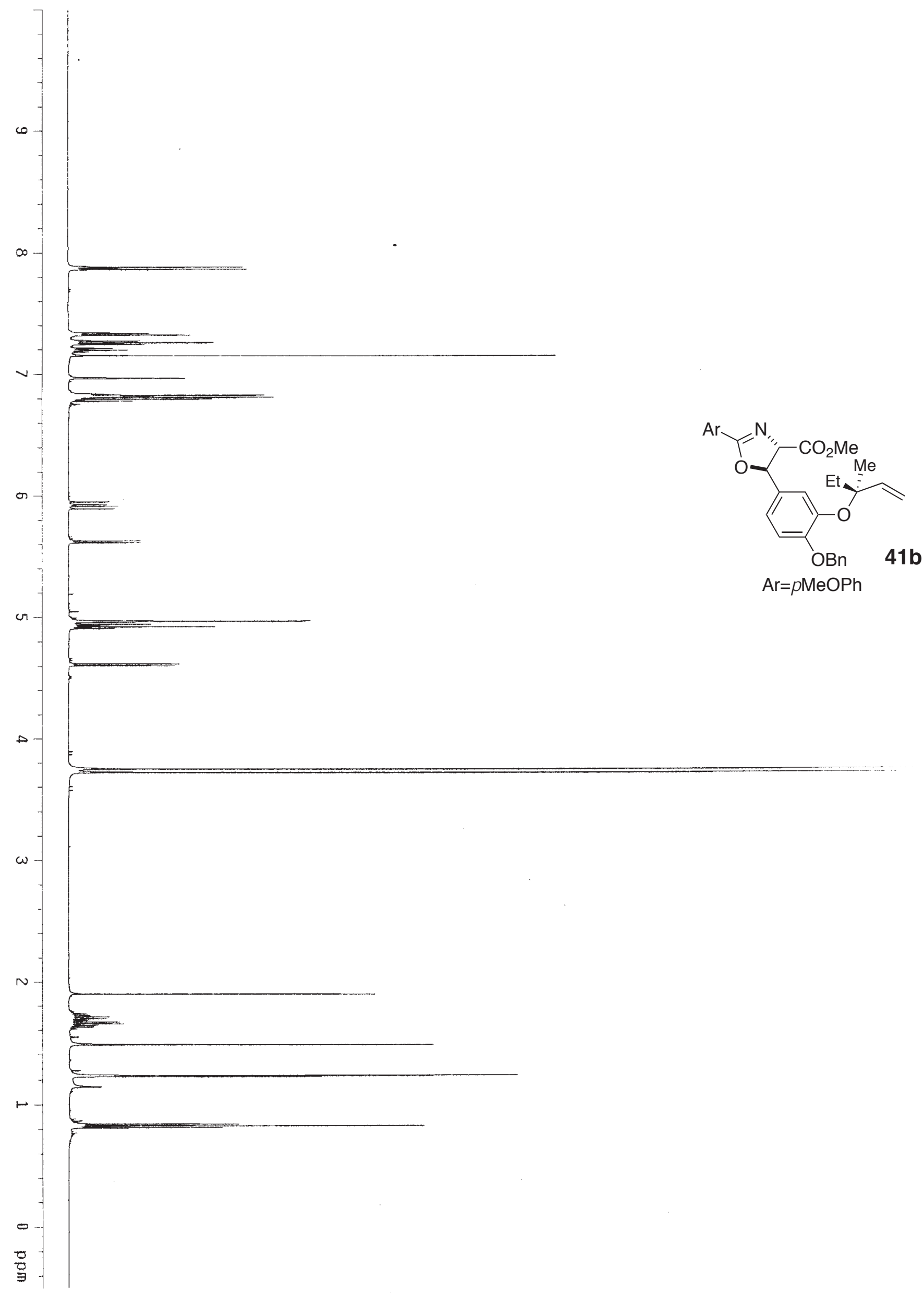




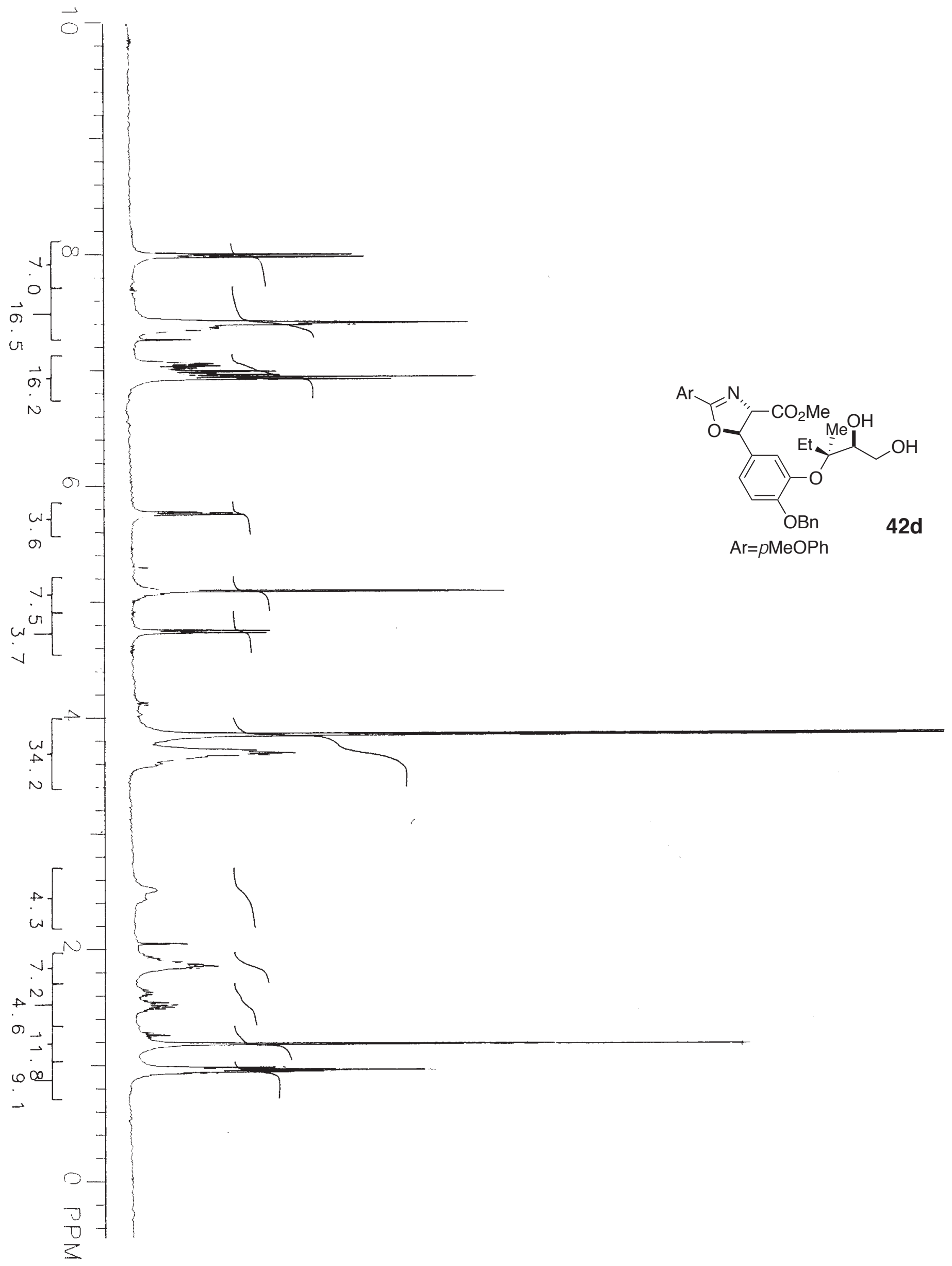




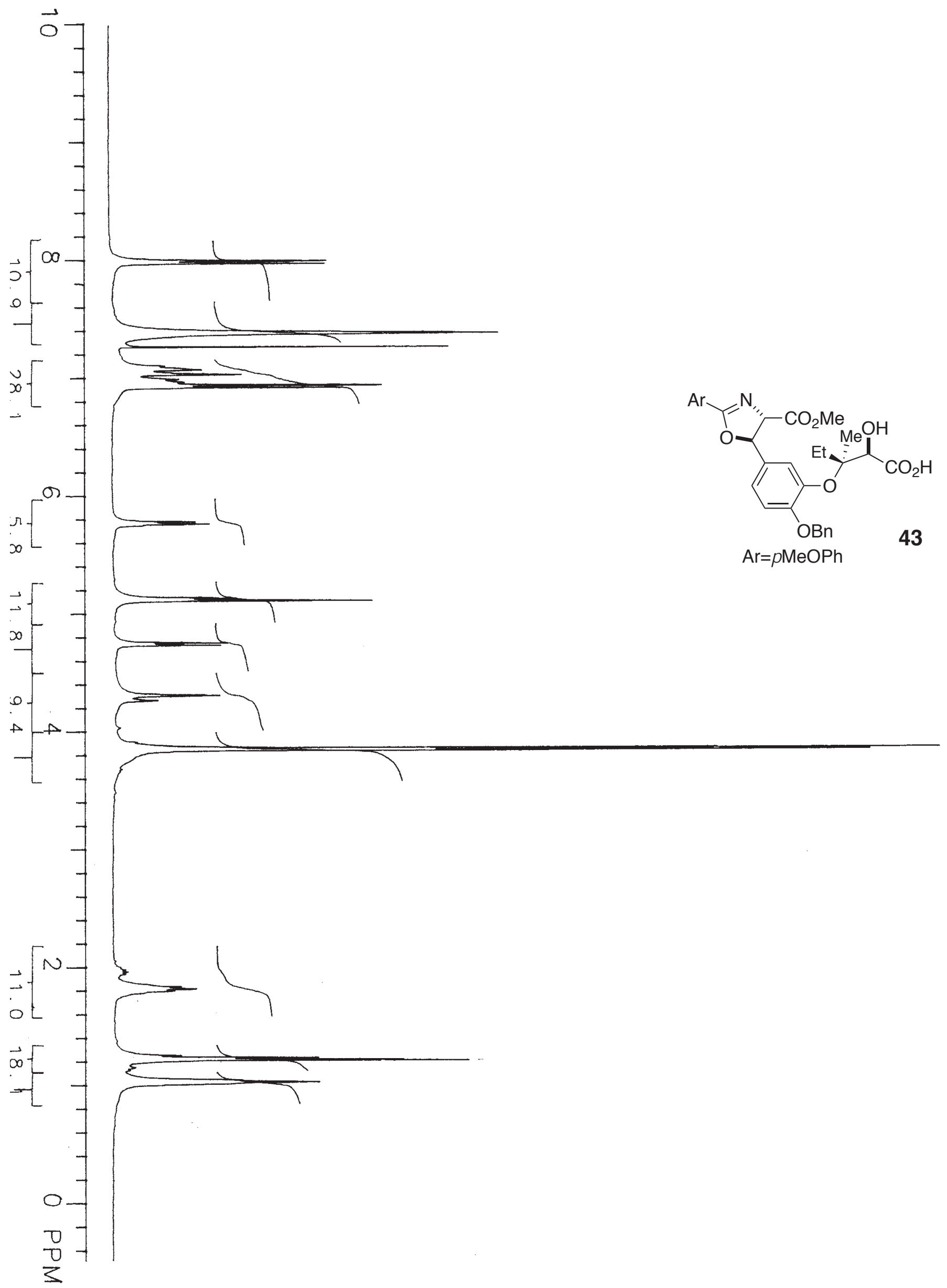




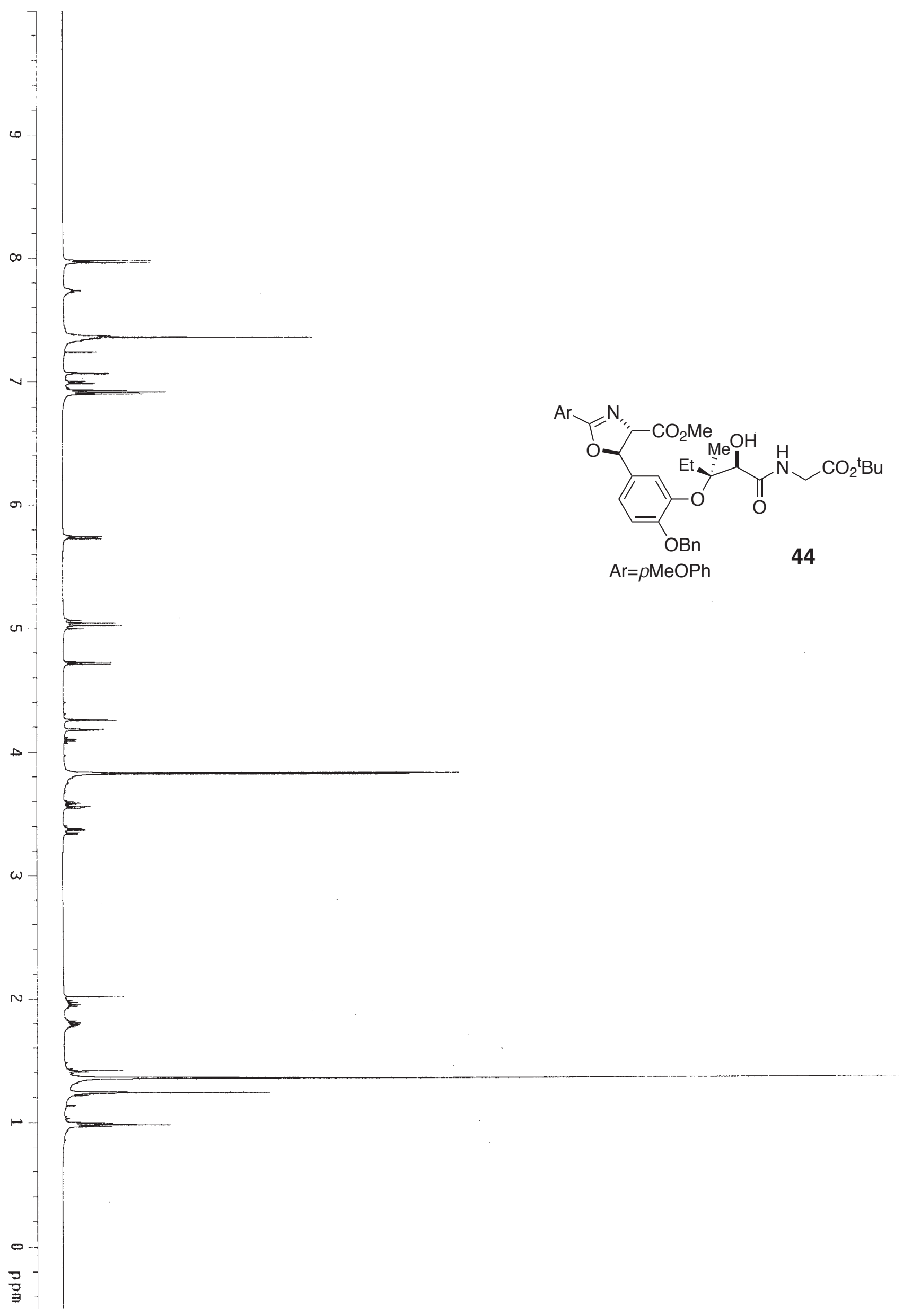


page S61

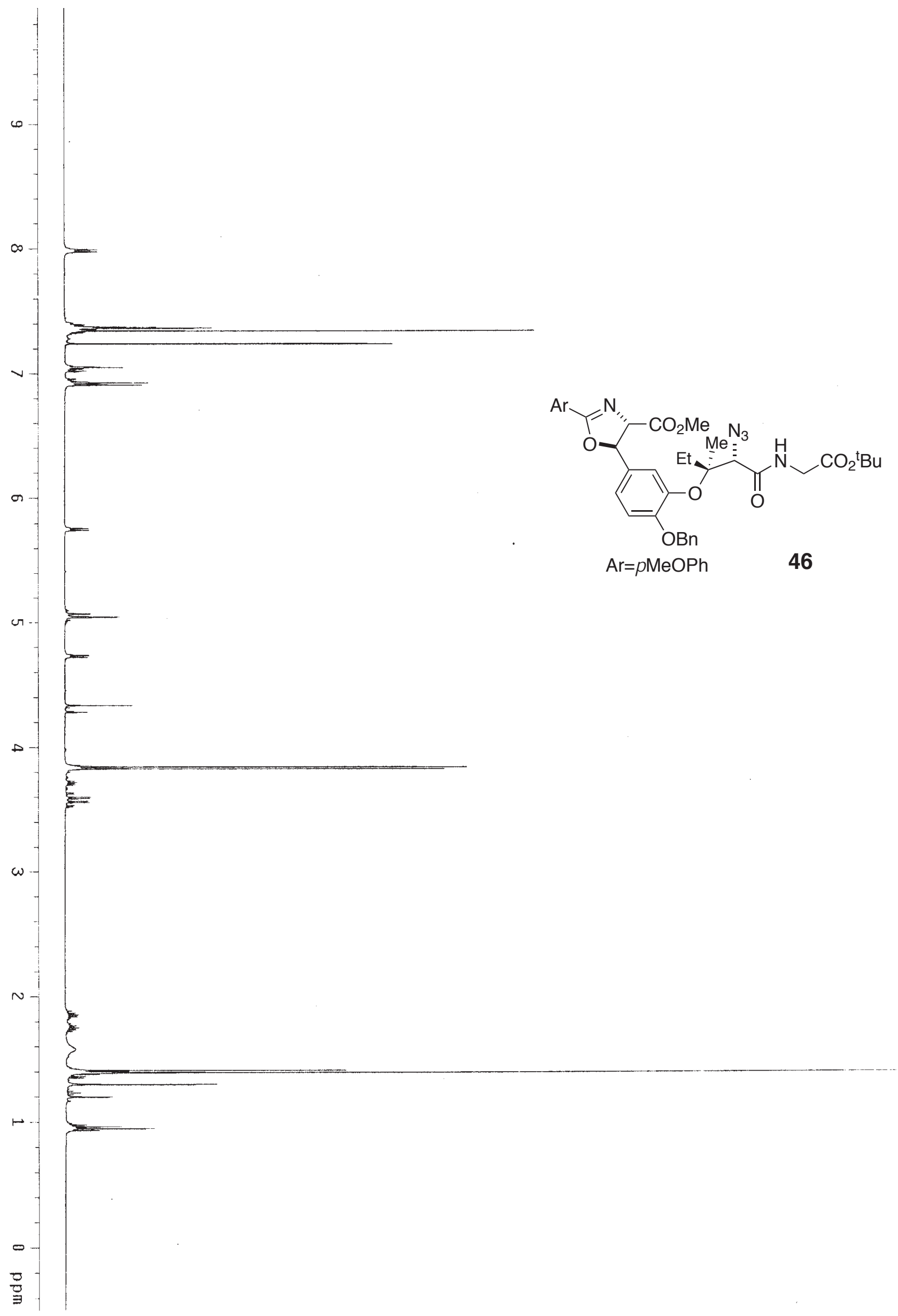




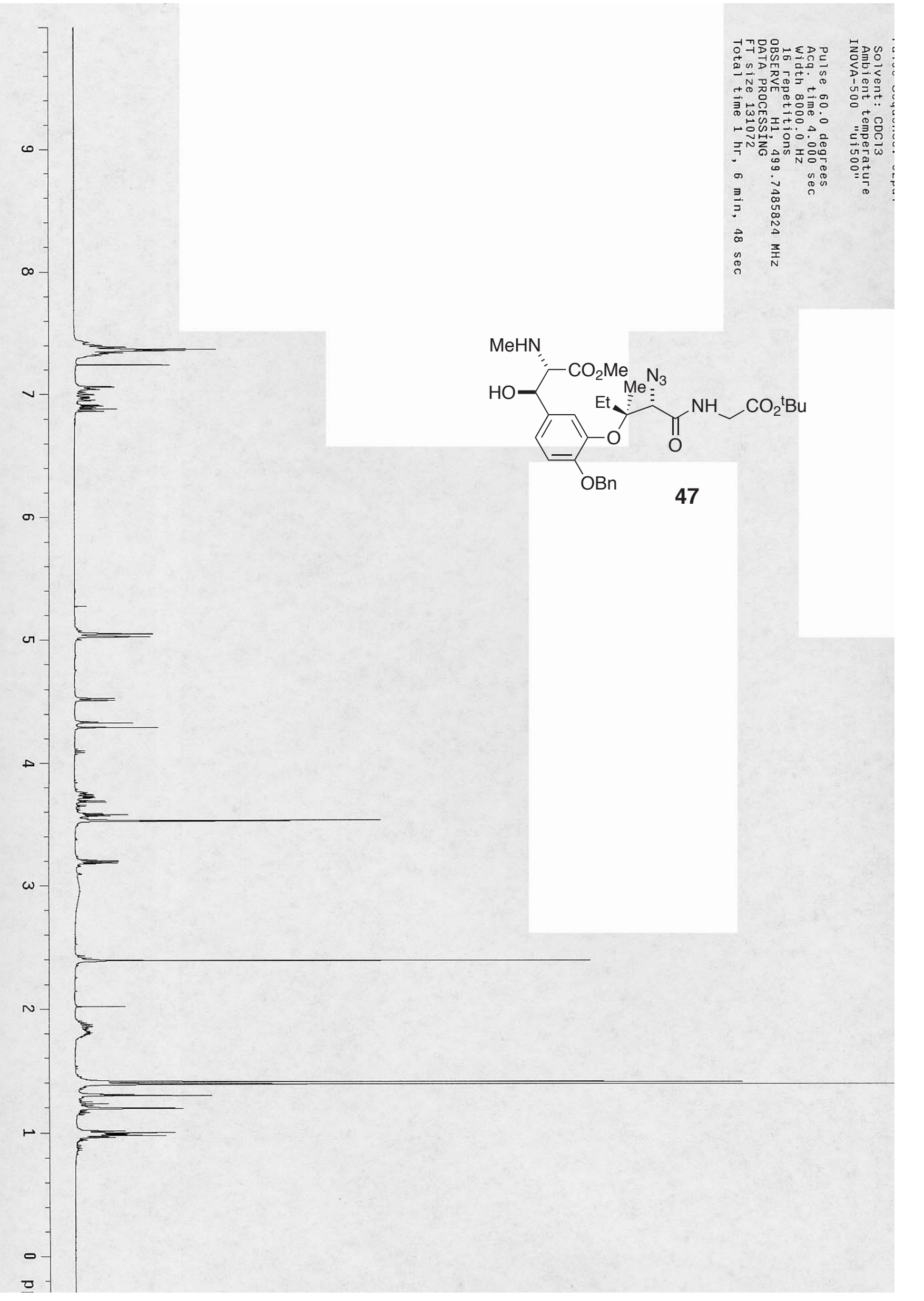




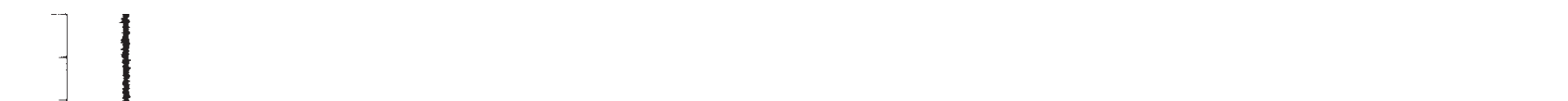<smiles>CCCCC(=O)OCc1ccc(C2OC([Al])=NC2C(C)=O)cc1OC(C)(N)C(N)C(=O)NCC(=O)Oc1ccccc1</smiles> 


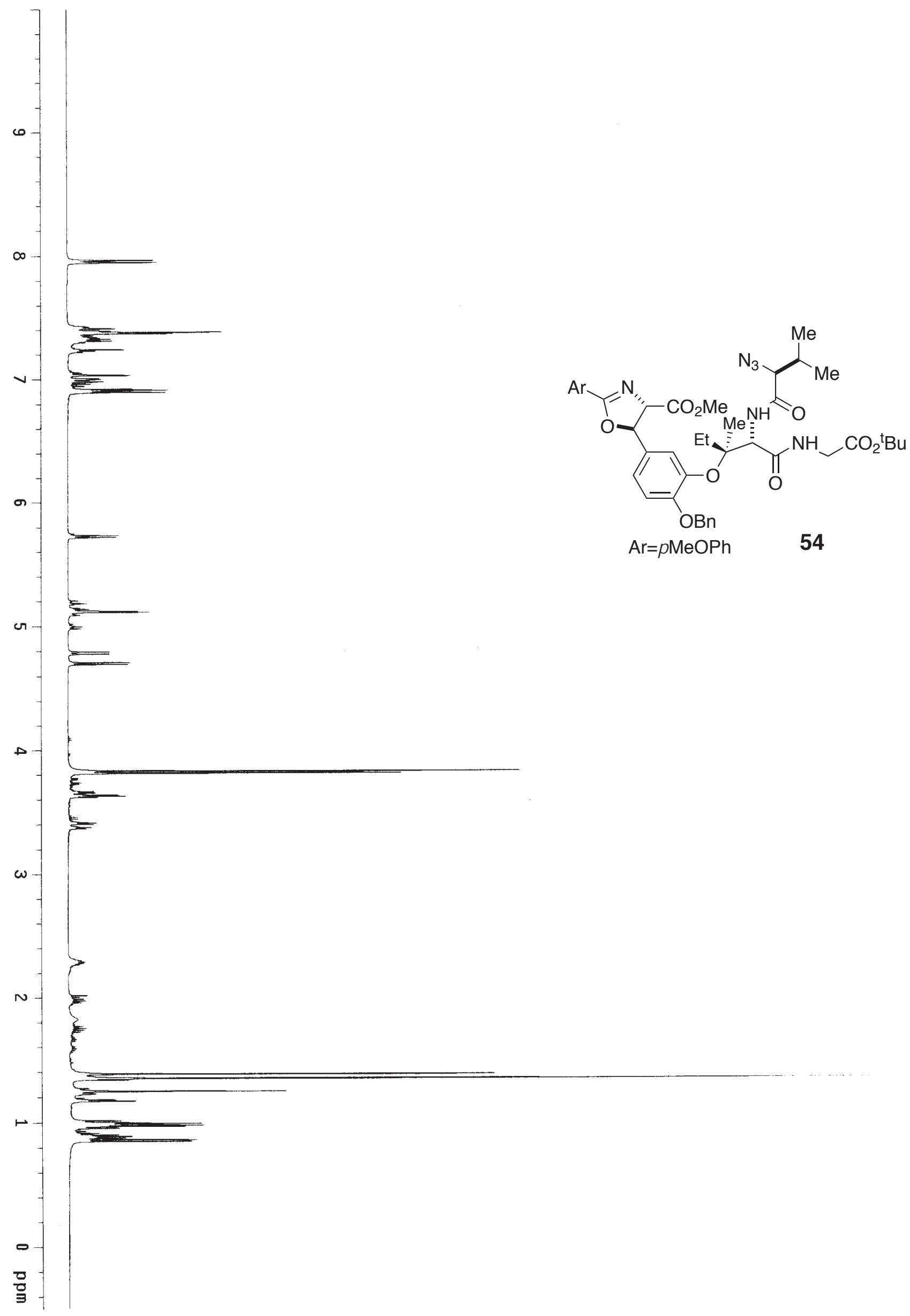


page S65

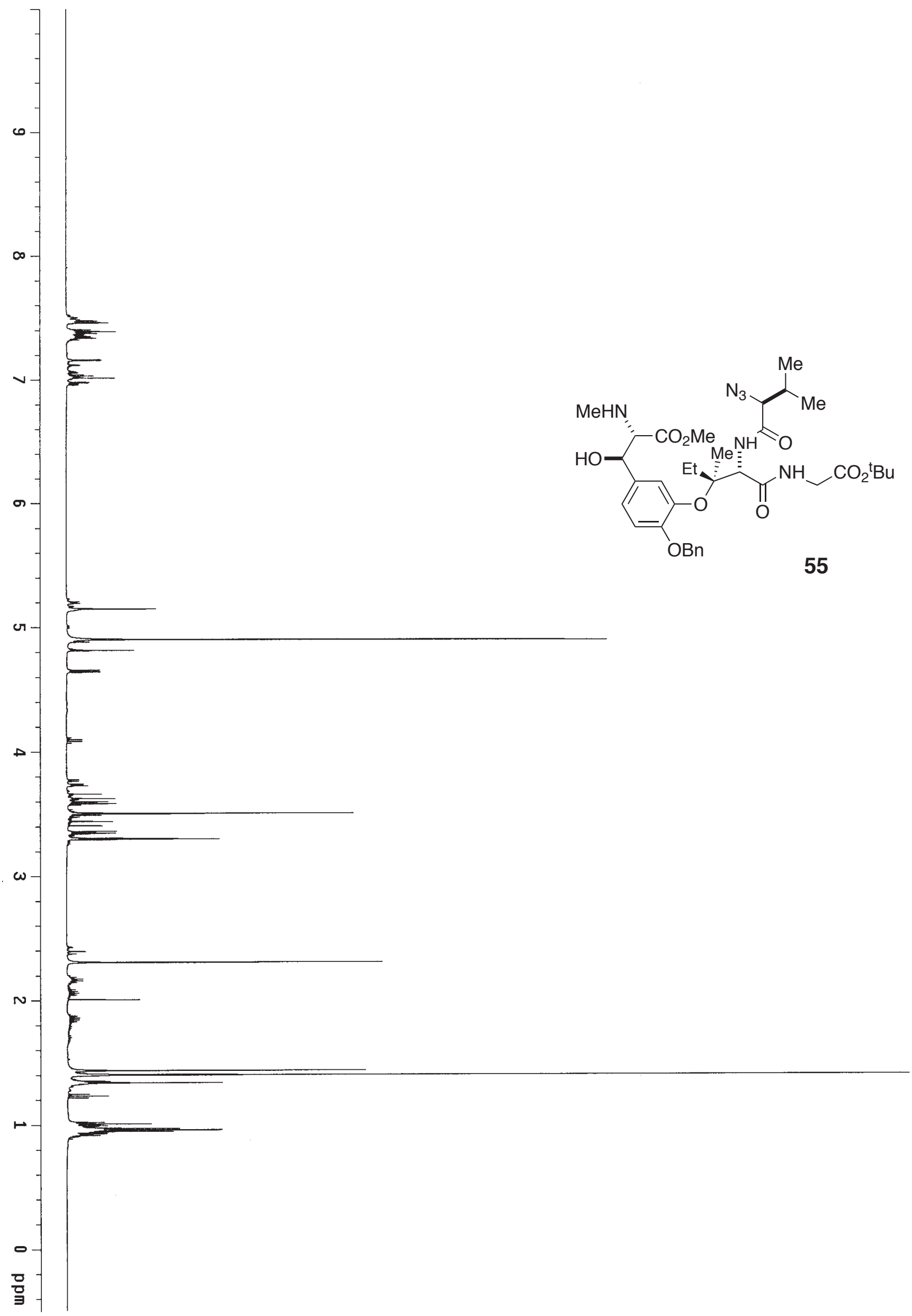




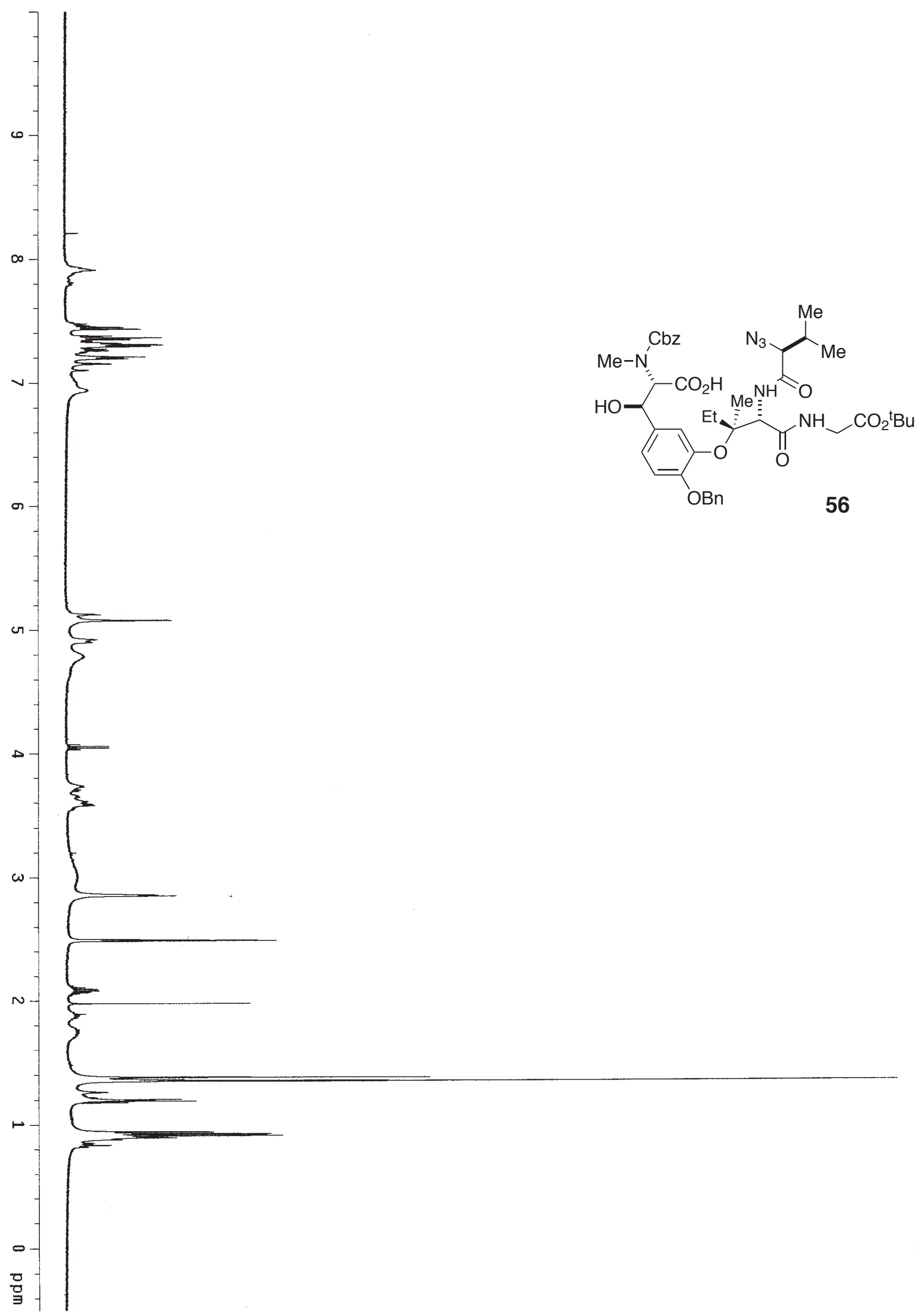




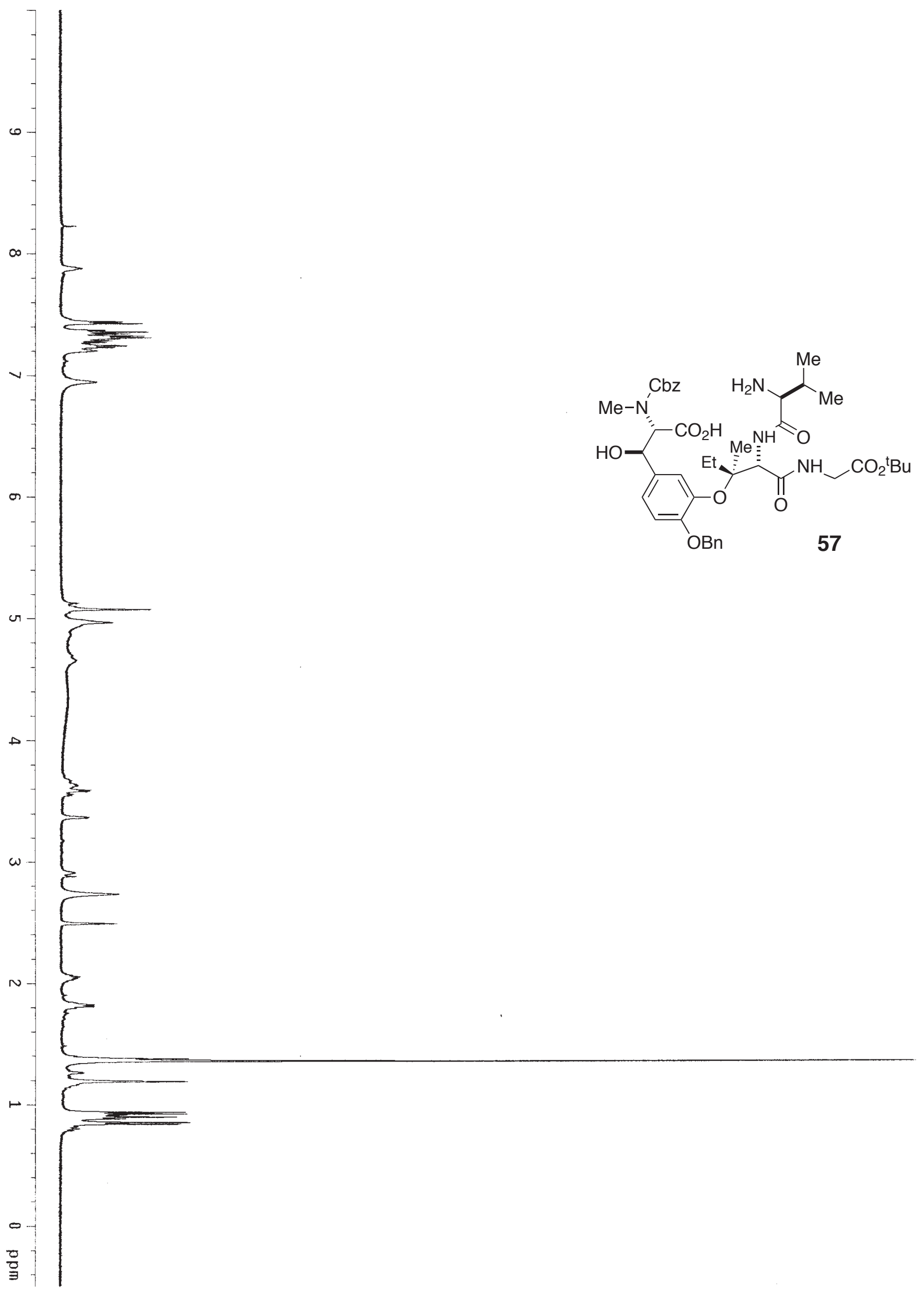




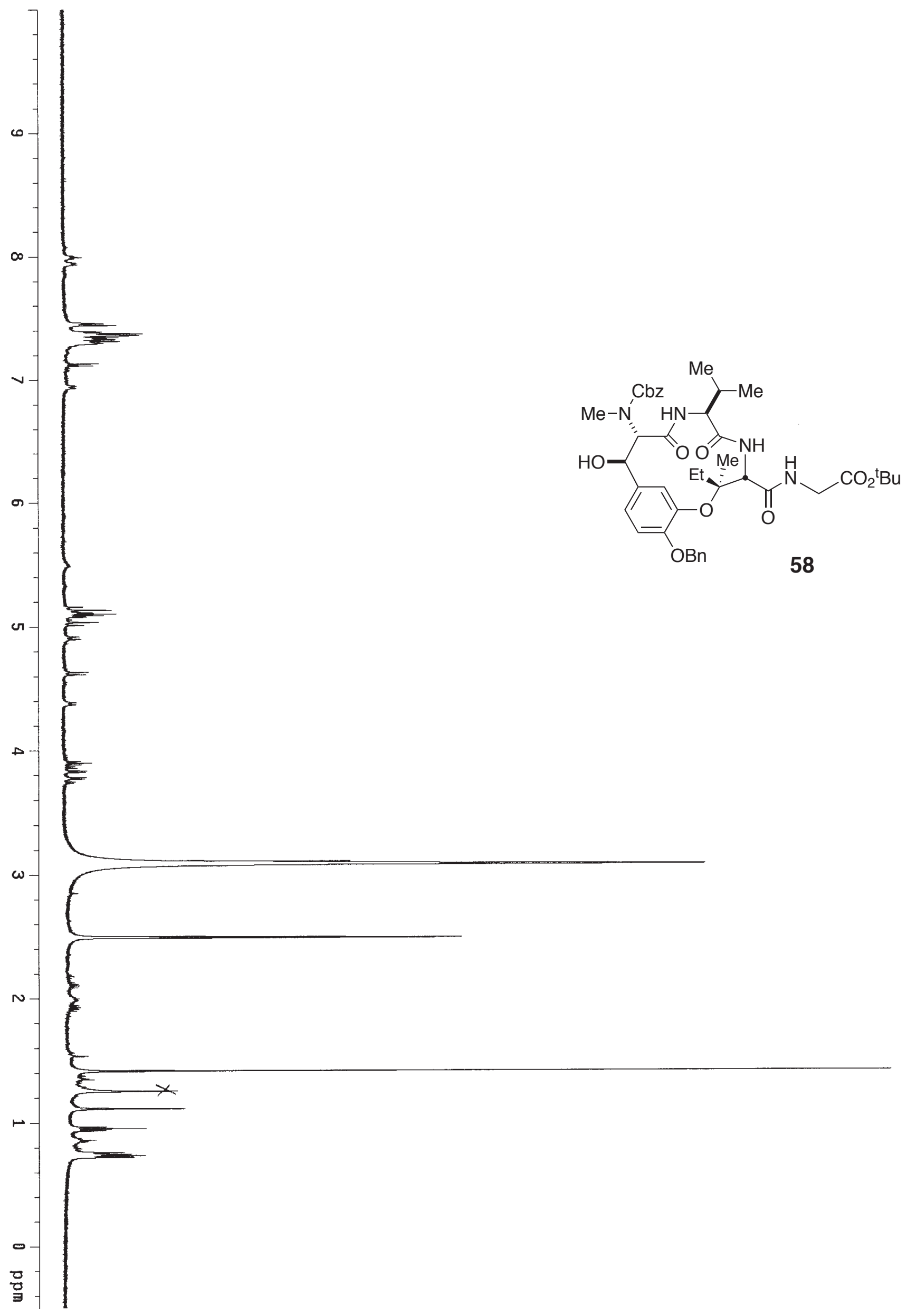




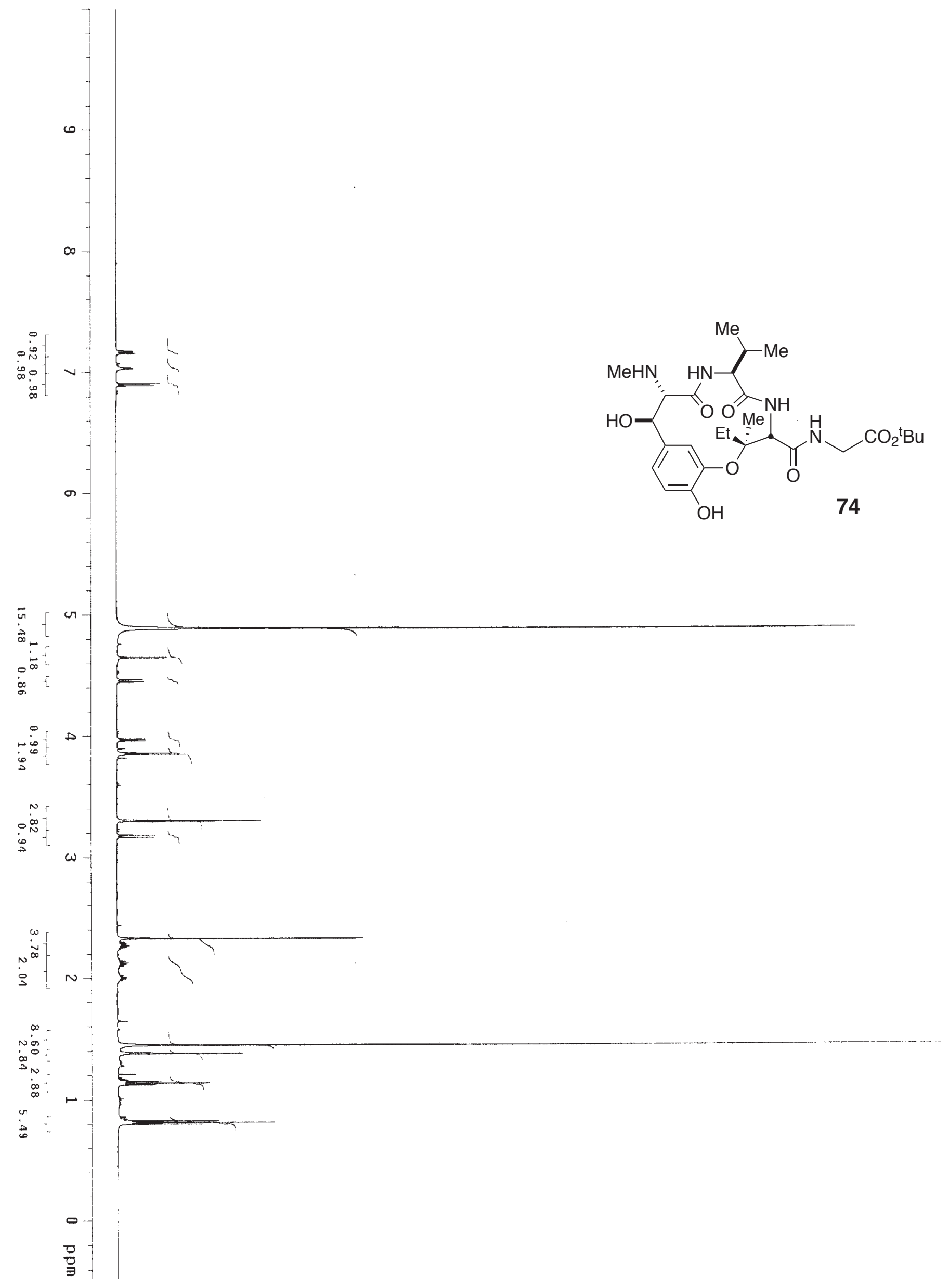




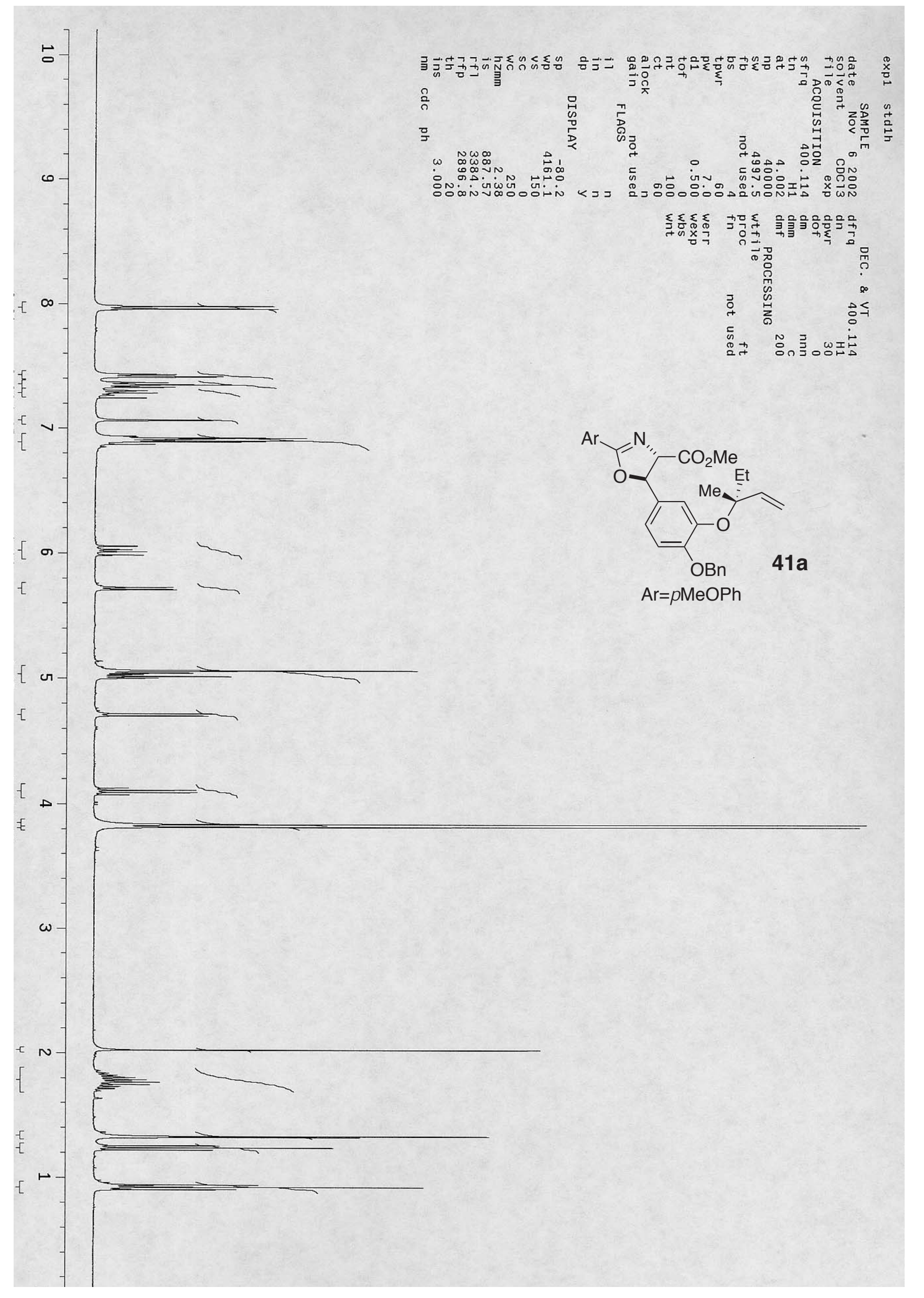




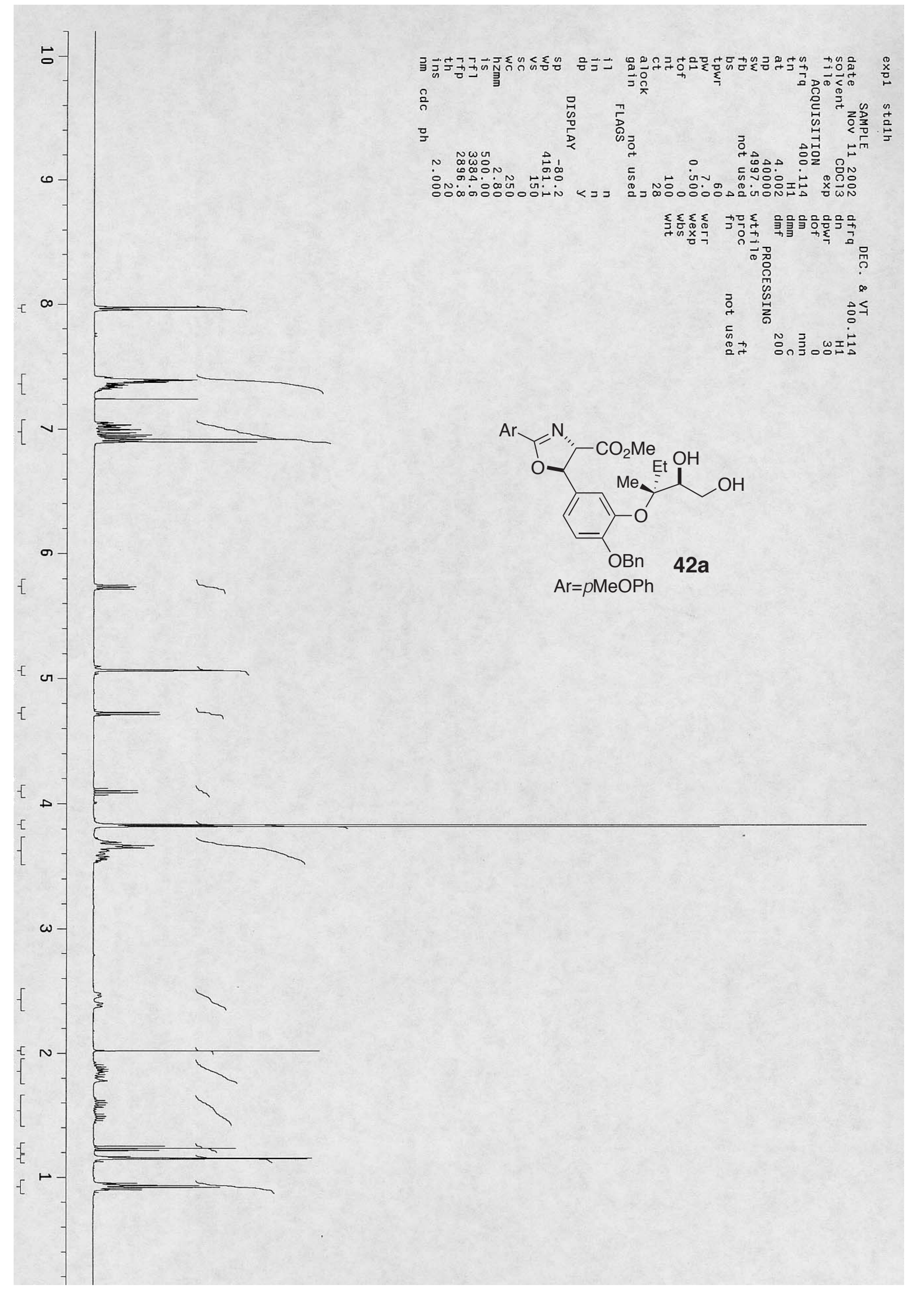




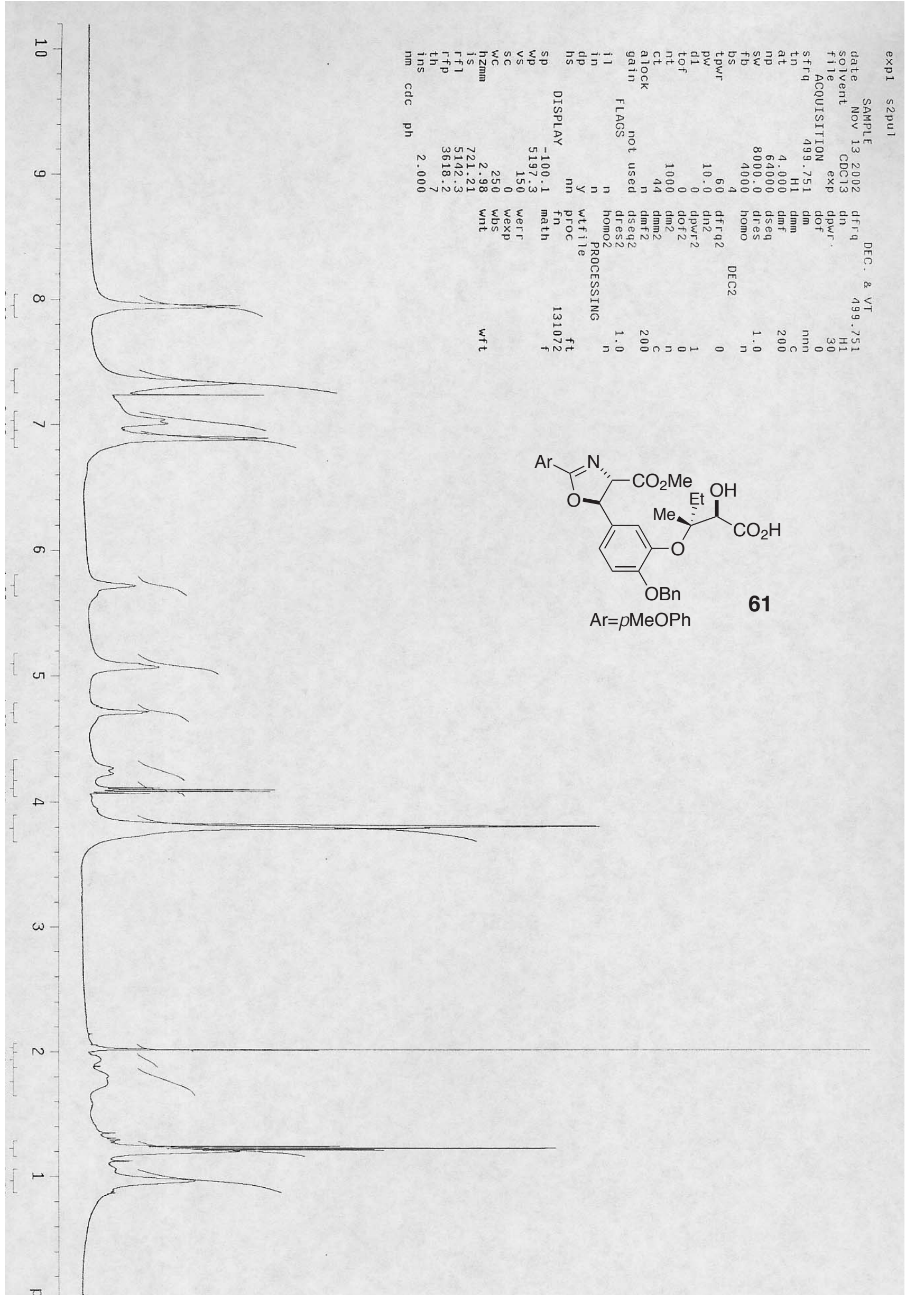




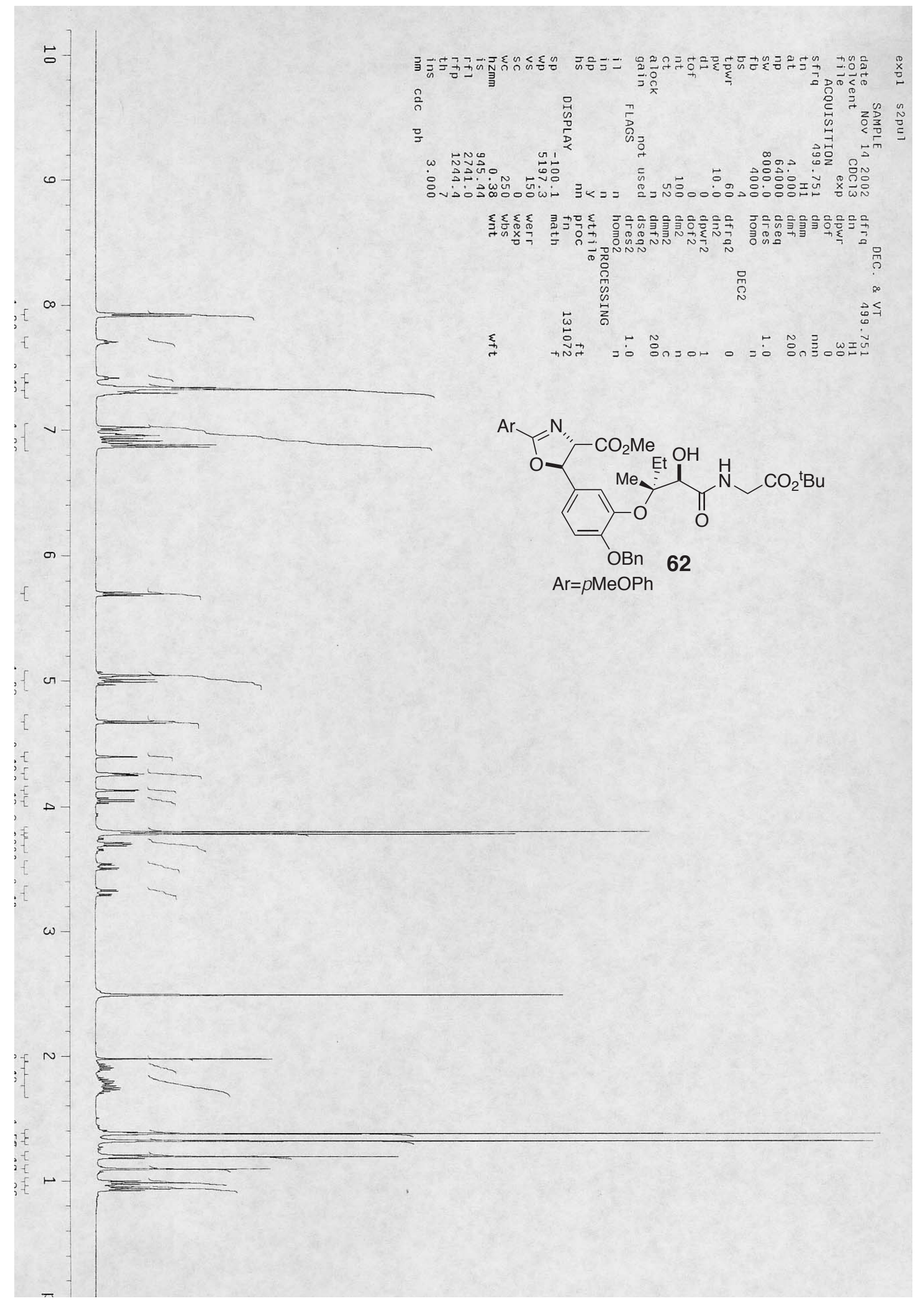




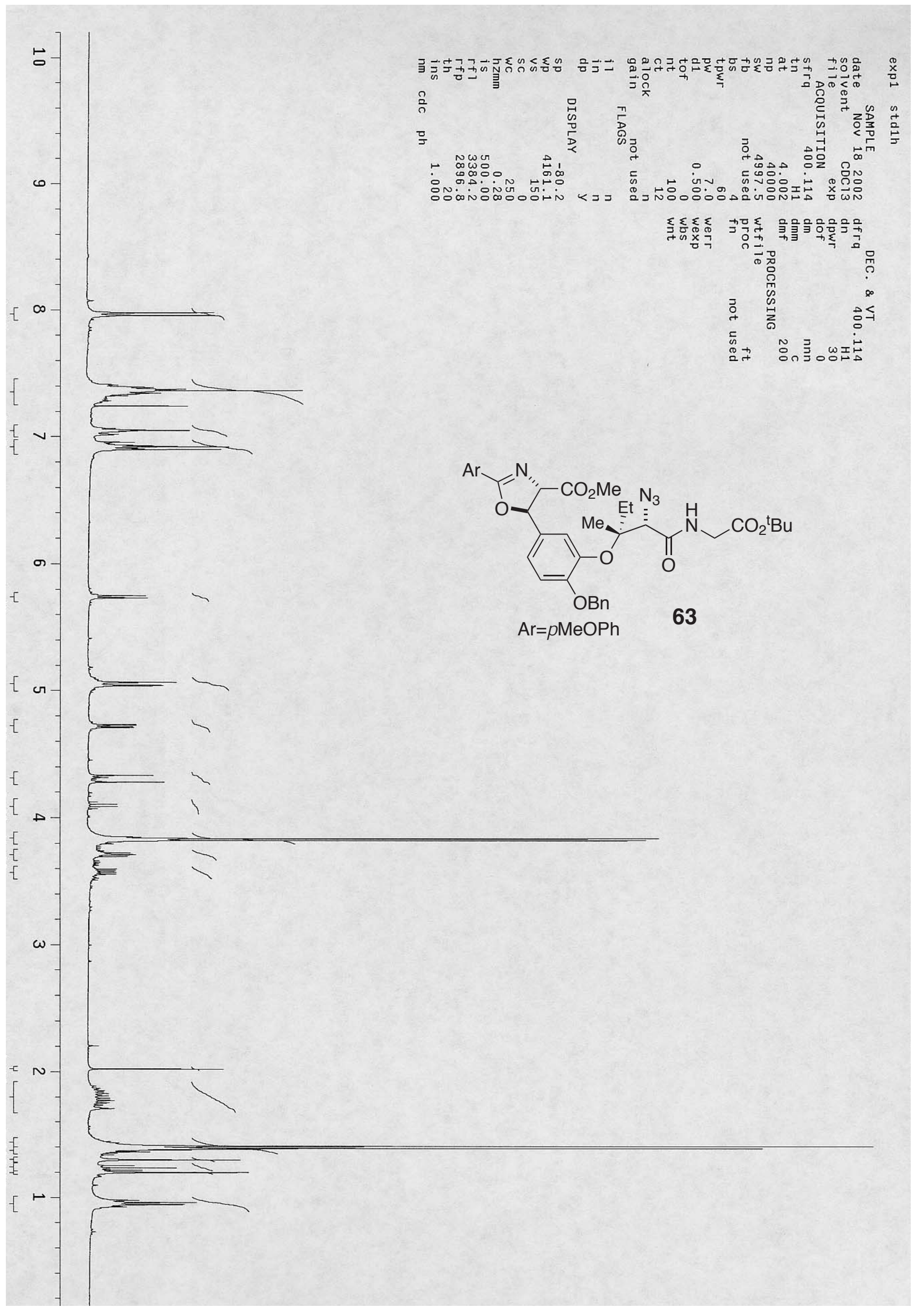




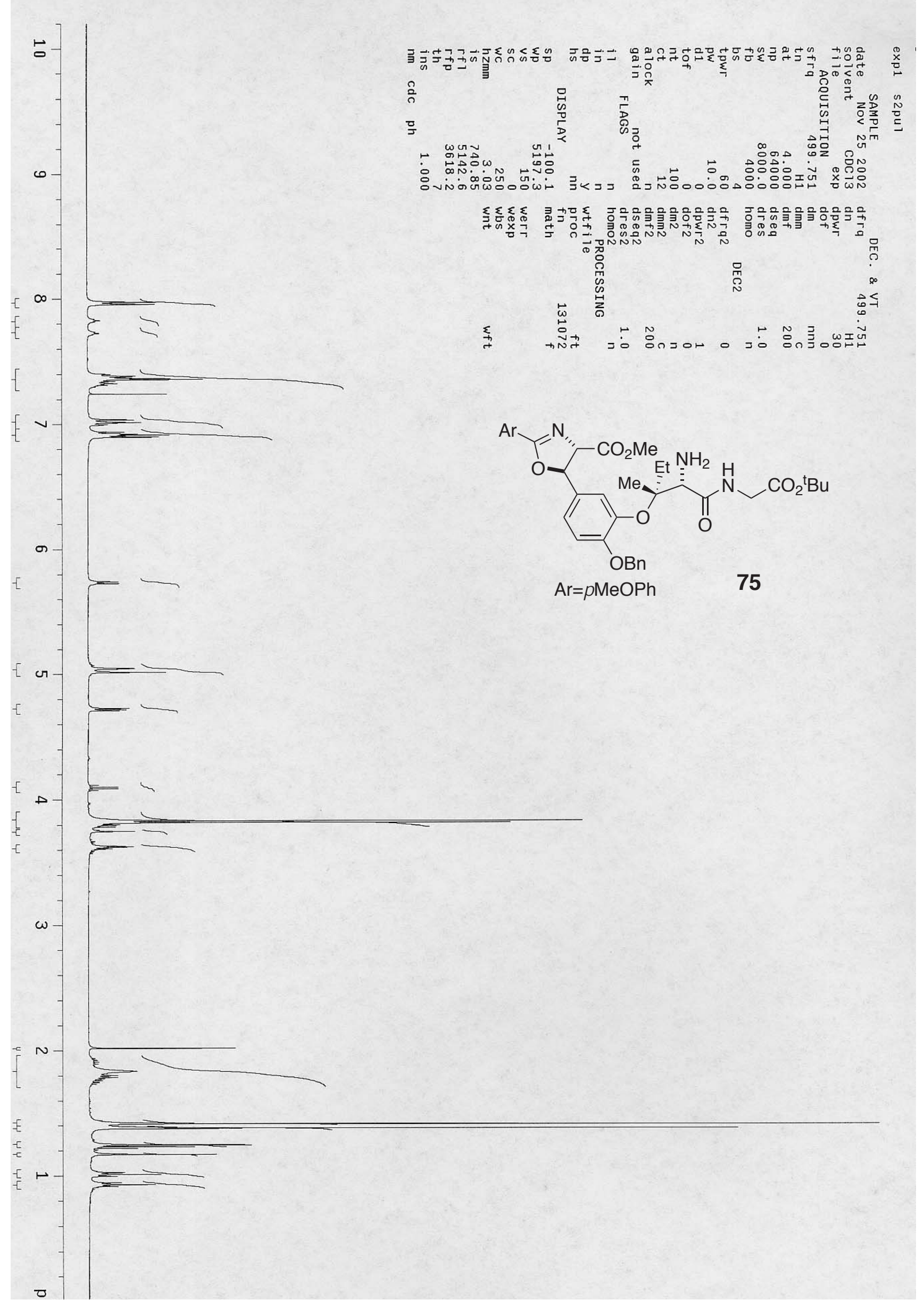




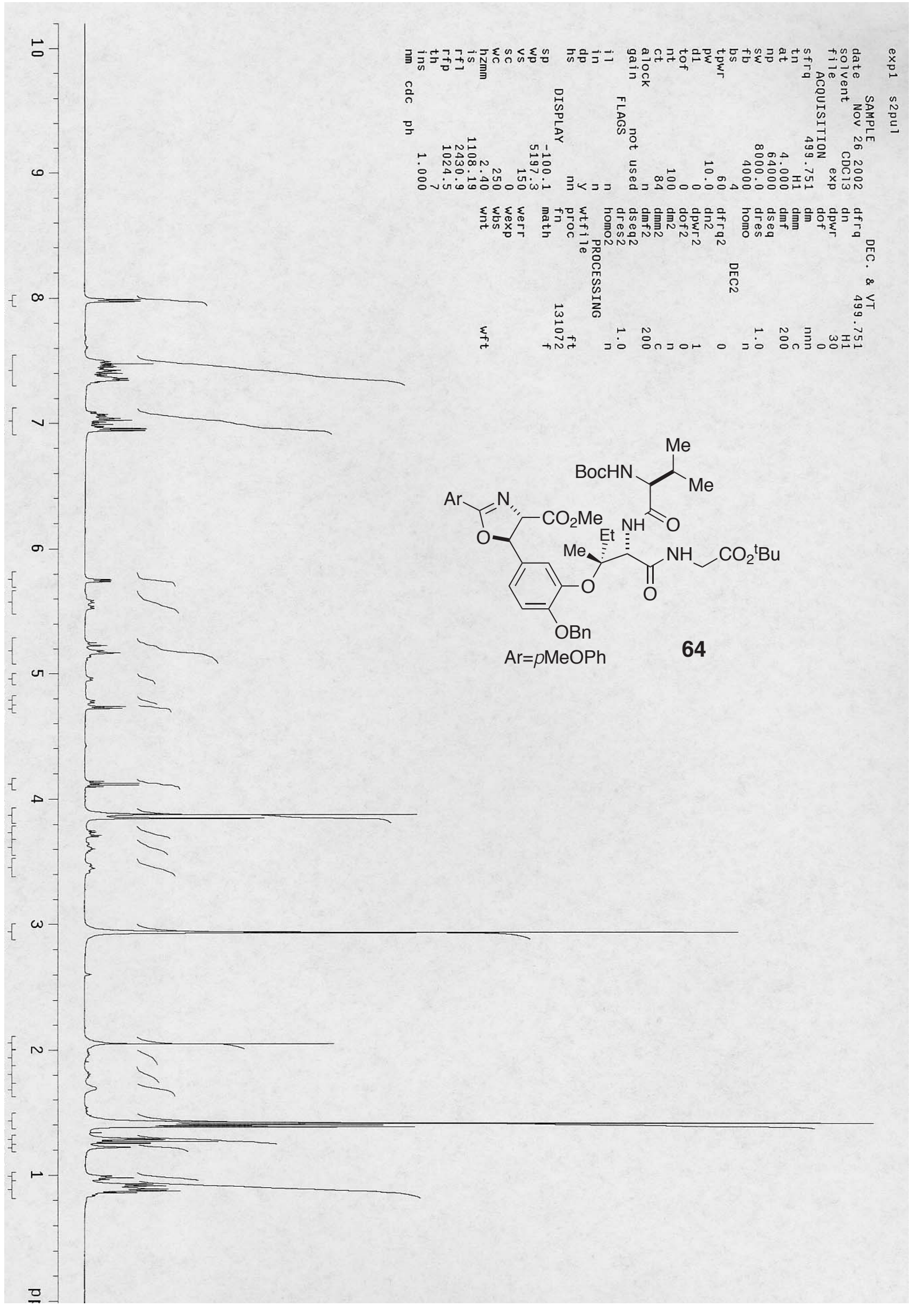




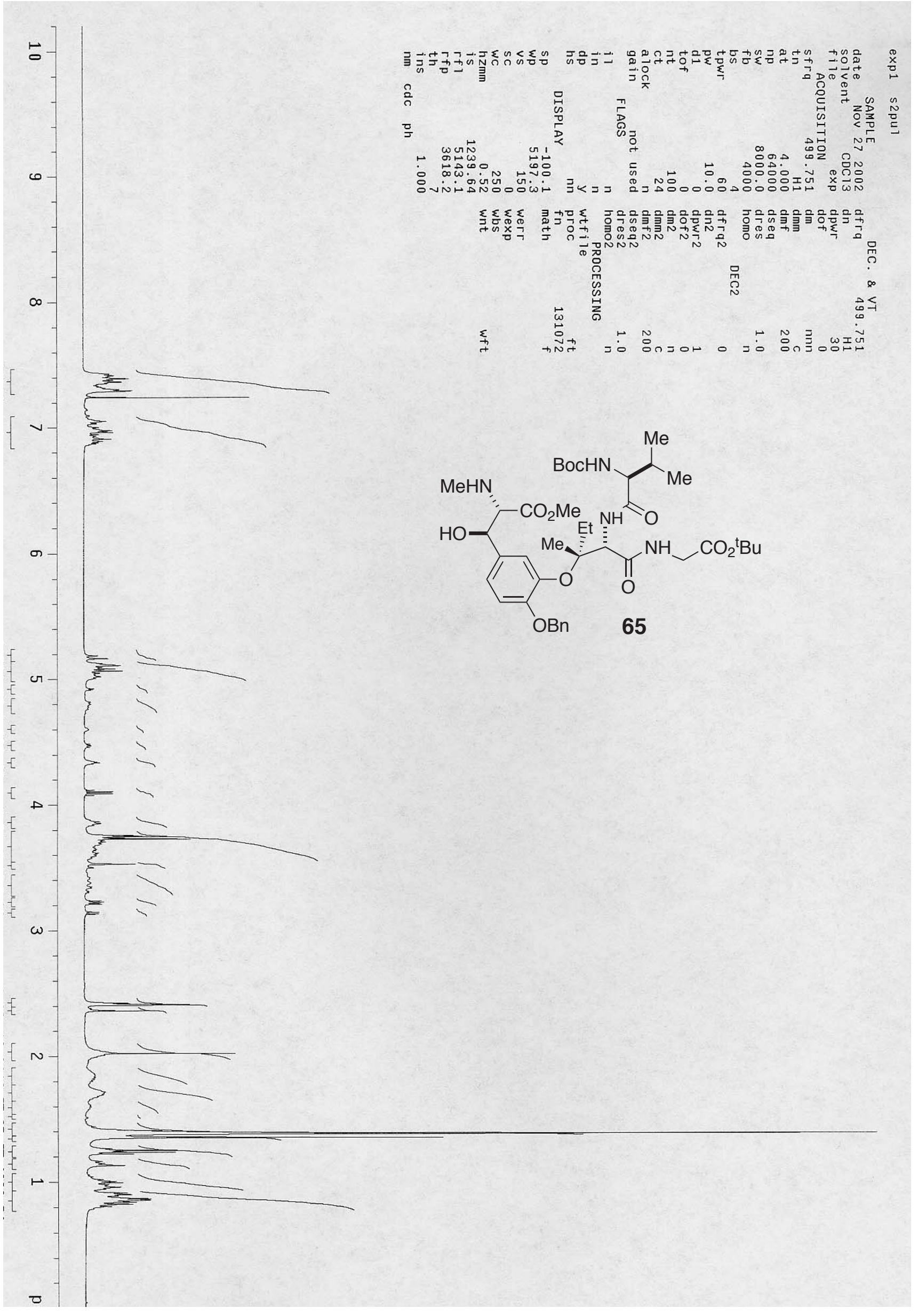




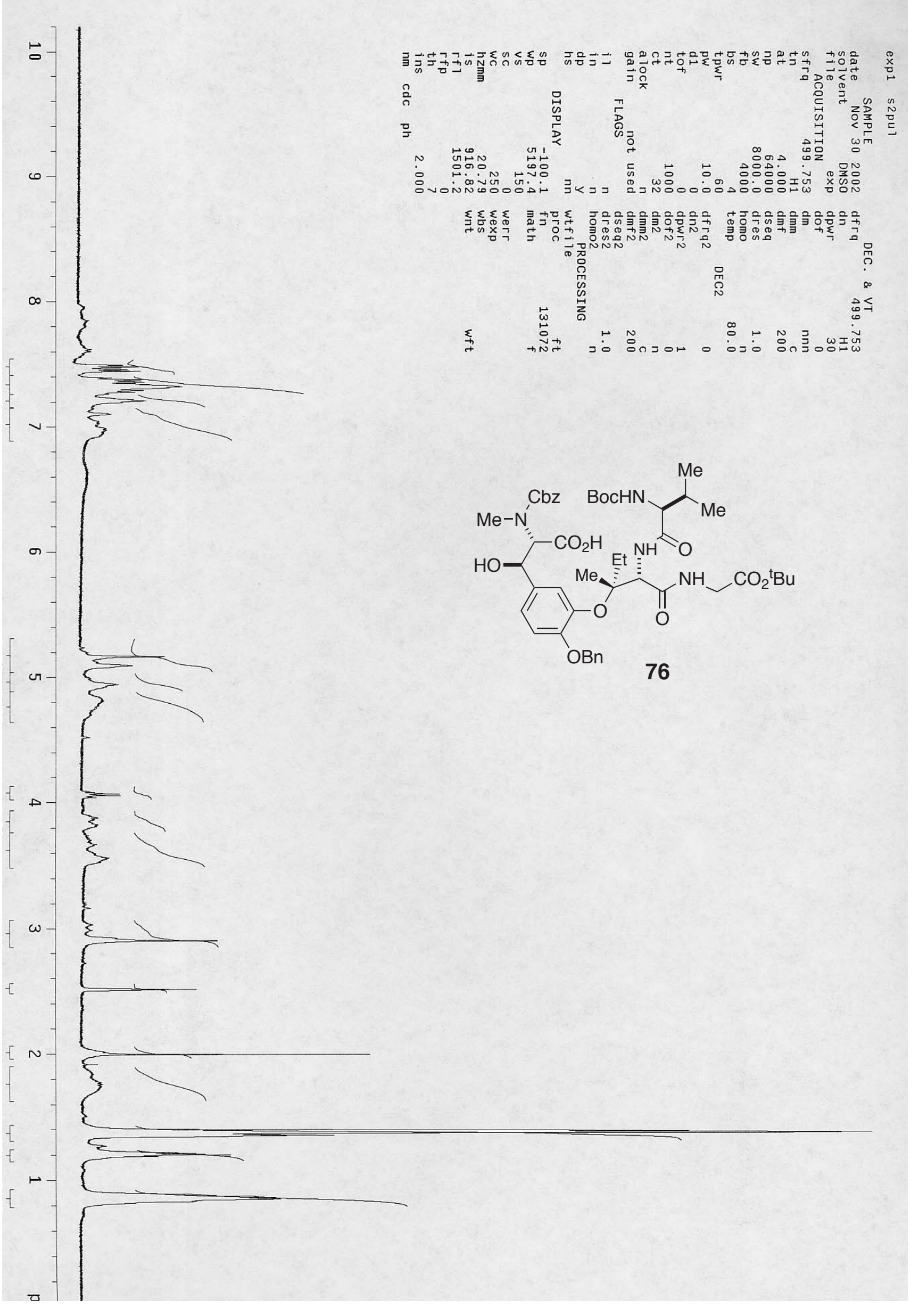




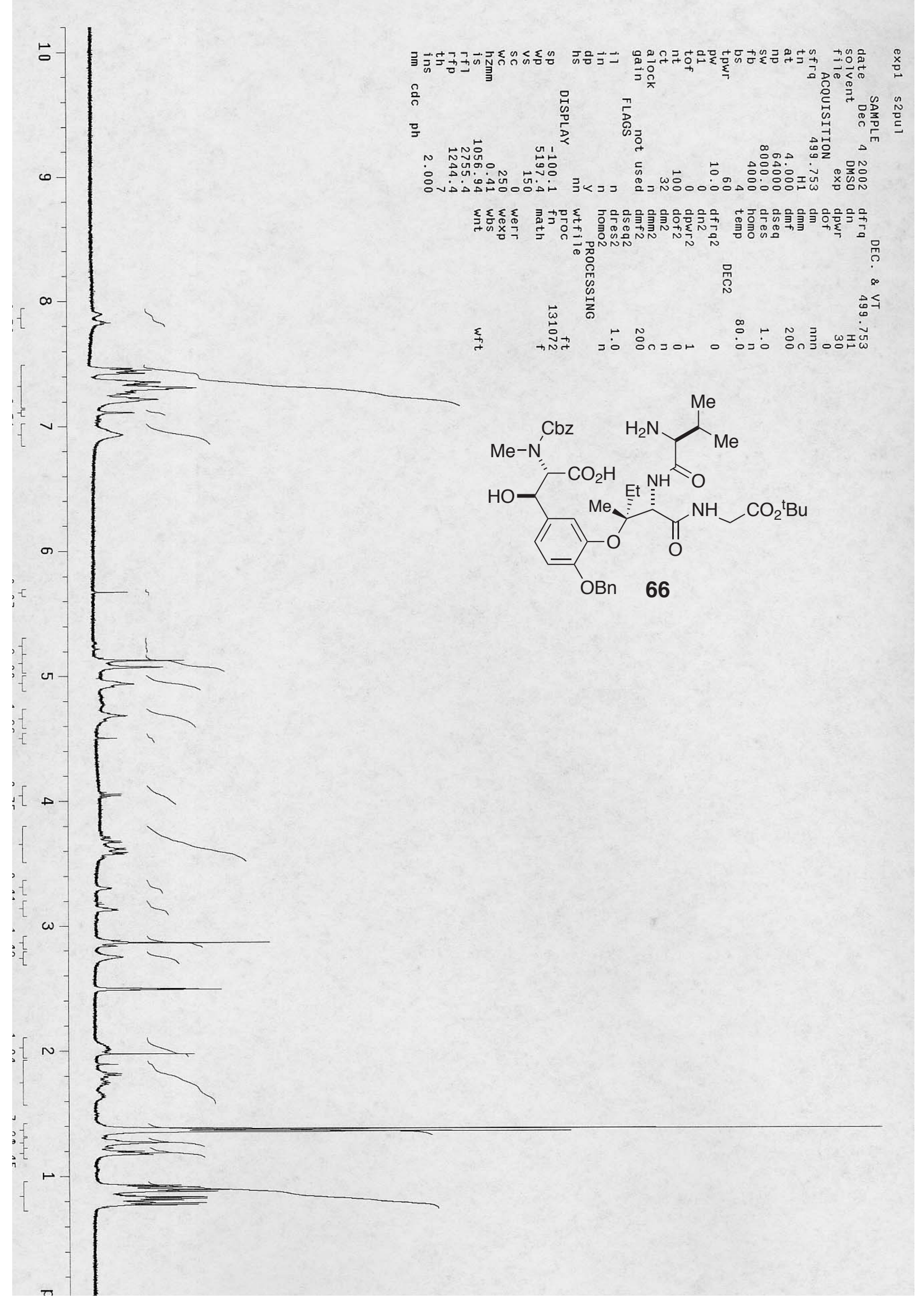




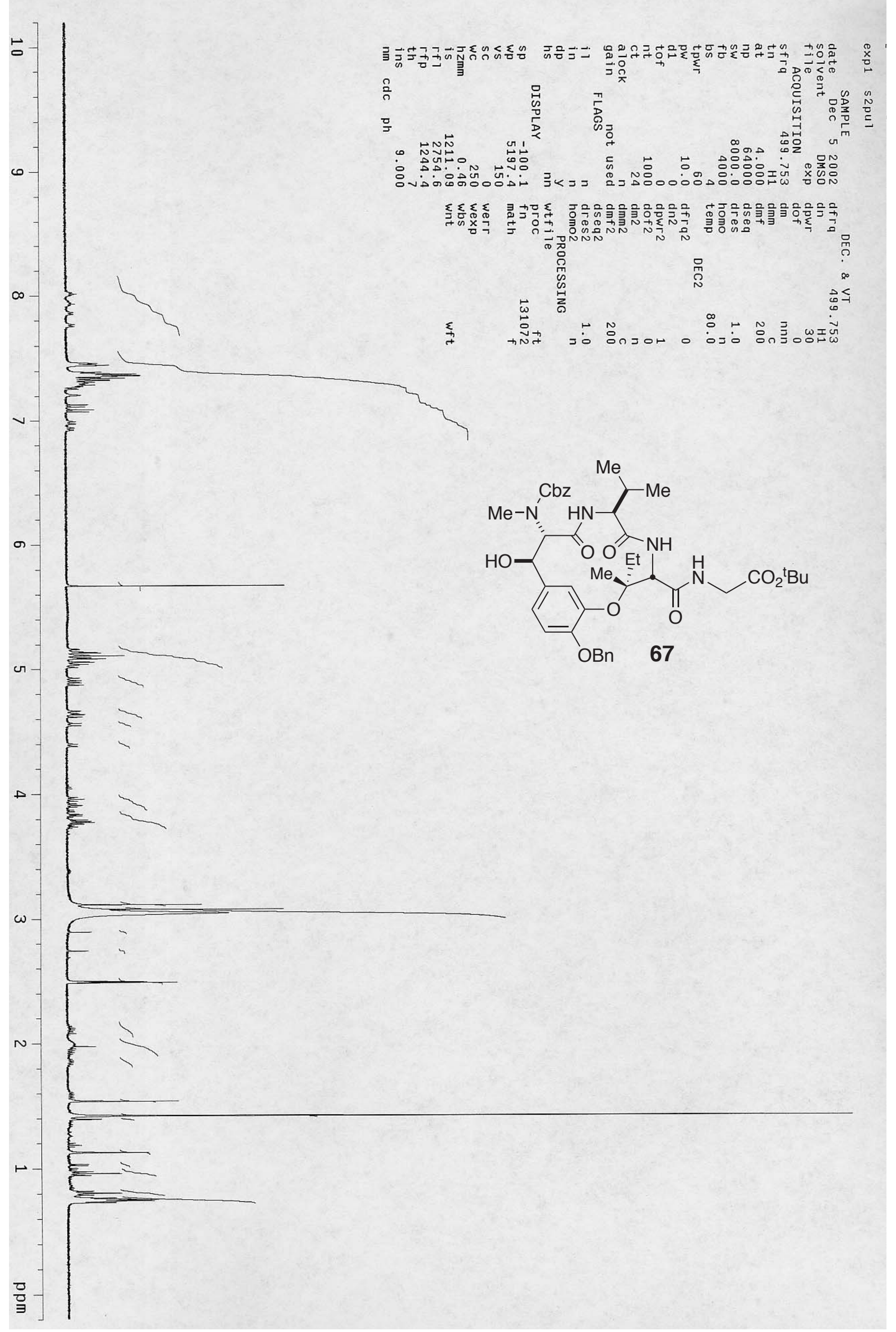




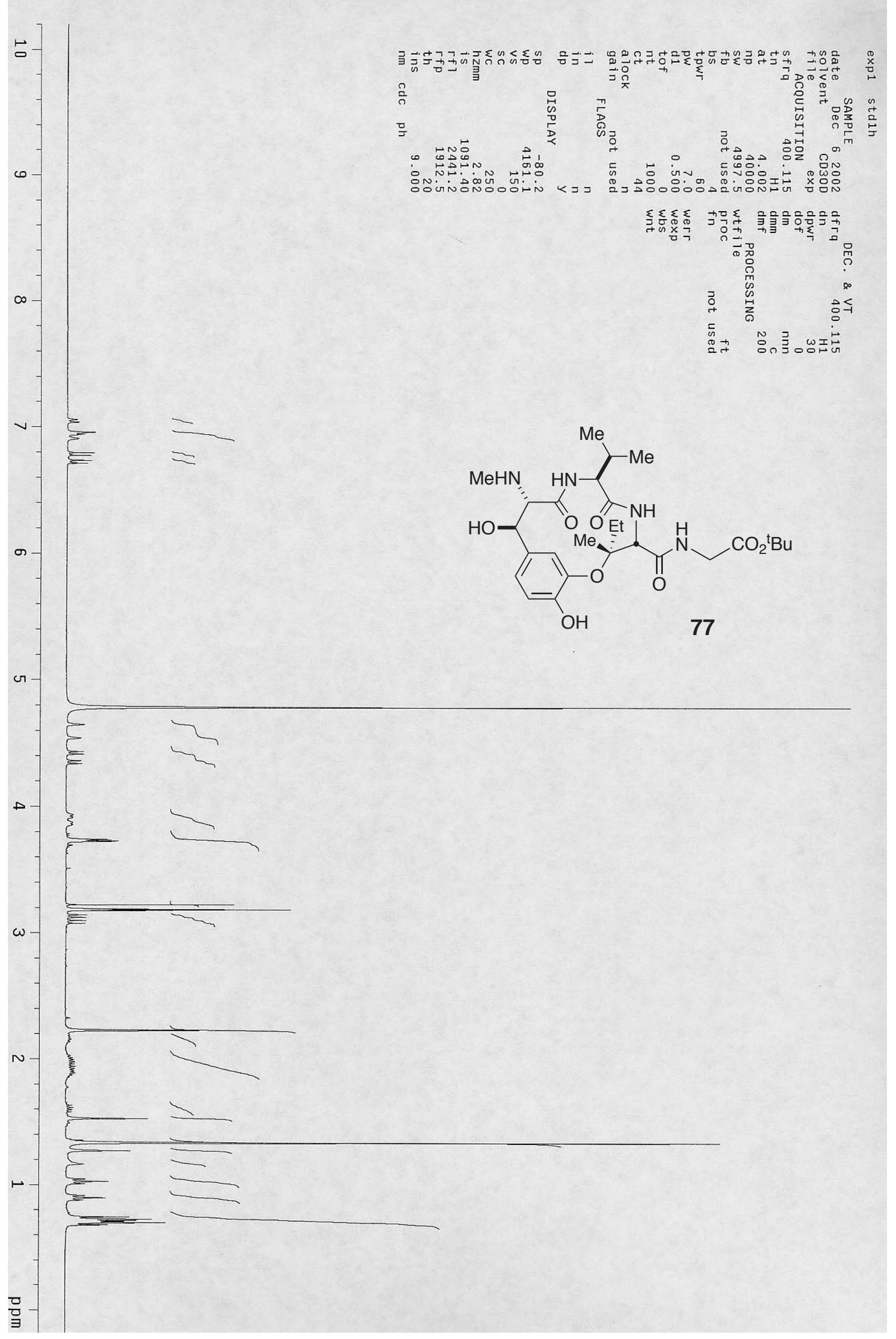

